\title{
Induced Compton-Scattering Effects in Radiation-Transport Approximations
}

Donald Richey Gibson, Jr.

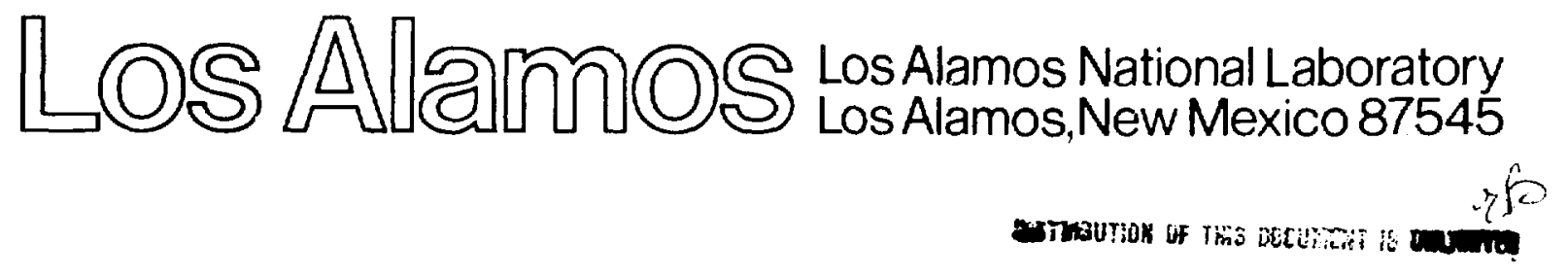




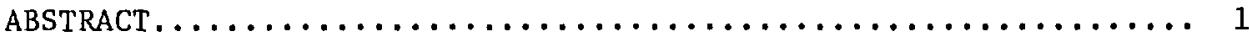

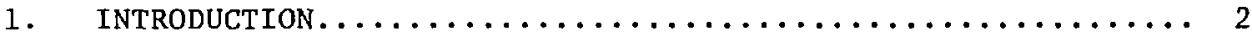

1.1 Objectives............................ 2

1.2 Review of Prior and Related Work............... 5

1.2.I Linear Radiation Transport............... 5

1.2.2 Nonlinear Radiation Transport............. 8

1.3 Summary of Thesis...................... 10

1.3 .1 General Methods..................... 10

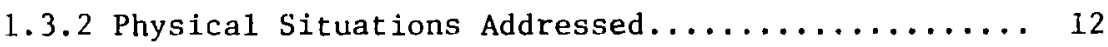

2. RAIIATION TRANSPORT EQUATIONS AND THEIR APPROXIMATIONS..... 17

2.1 The Linear Radiation Transport Equation............ 17

2.2 The Nonlinear Radiation Transport Equation.......... 21

2.3 Approximation of the Radiation Transport Equation...... 32

2.3 .1 Diffusion Theory..................... 33

2.3 .2 Transport Approximations................ 40

3. METHOD OF CHARACTERISTICS IN RADIATION TRANSPORT........ 61

3.1 Reduction to Canonical Form................. 61

3.2 General Method.......................... 73

3.3 Eraluation of the Scattering Kernel............. 80

4. NUMERICAL RESULTS FOR SPATIALLY DEPENDENT AND SPATIALLY

INDEPENDENT RADIATION TRANSPORT PROBLEMS............. 91

4.1 Spatially Independent Solutions................. 93

4.2 Spatially Dependent Solutions................. 118

5. CONCLUSIONS AND RECOMMENDATIONS FOR FURTHER STUDY........ 122

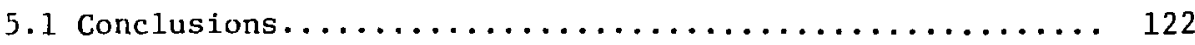

5.2 Recommendations for Further Study.............. 125

ACKNOWLEDGMENTS ............................ 127

REFERENCES $\ldots \ldots \ldots \ldots \ldots \ldots \ldots \ldots \ldots \ldots \ldots \ldots \ldots \ldots \ldots \ldots \ldots \ldots \ldots \ldots \ldots$

APPENDIXES

I. REDUCTION OF FIRST ORDER PARTIAL DIFFERENTIAL

EQUATIONS TO CANONICAL FORM.................. 132

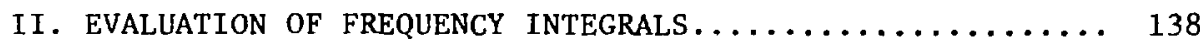

III. GENERAL TRANSPORT CODE.................... 140 
INDUCED COMPTON SCATTERING EFFECTS IN RADIATION

TRANSPORT APPROXIMATIONS

by

Donald Richey Gibson, Jr.

ABSTRACT

In this thesis the method of characteristics is used to solve radiation transport problems with induced Compton scattering effects included.

The methods used to date have only addressed problems in which either induced Compton scattering is ignored, or problems in which linear scattering is 1gnored. Also, problems which include both induced Compton scattering and spatial effects have not been considered previously. The introduction of induced scattering into the radiation transport equation results in a quadratic nonlinearity. Methods are developed to solve problems in which both linear and nonlinear Compton scattering are iuportant. Solutions to scattering problems are found for a variety of initial photon energy distributions. 


\section{INTRODUCTION}

\subsection{Objectives}

Radiation transport is a topic which has been investigated for a large number of physically interesting situations. The study of radiative transfer in a stellar medium has been of interest to astrophysical investigators for many years.(1) The subject of radiation transport has a more recent application in the development of laser driven fusion. One area of interest here is the coupling of laser radiation to a small fusion target. Another area where proper radiation transport treatment is essential is during the burn of a snall fusion target. Radiation produced in the burning plasma must interact with surrounding material and is a major mode of energy transport through Compton and inverse Compton processes.

Induced Compton and Inverse Compton scattering is not included in many of the methods currently in use for radiation transport. The variable Eddington method $(2,3,7)$ is used extensively. This method does not include induced scattering. The transport approach of Freeman and Davis(5) neglects induced scattering also. Monte Carlo approaches to radiation transport do not include induced scattering. (4) Inclusion of Induced effects in Monte Carlo schemes is discussed in Section 1.2.1. The approaches of Zel'dovich $(11,13)$ and 
Montes $(14,38)$ treat induced scattering in spatially independent problems but neglect linear scattering terms.

The treatment of induced Compton and inverse Compton scattering In transport approximations is the central topic of this thesis. Both linear and nonlinear scattering cerms are included. An electron gas is the transport medium addressed in this thesis. Consequently, no absorption or enission can occur.

The physical interpretation of induced processes can be stated quite simply $(6)$. This effect applies for all bosons. Hence, sixce photons are bosons, this effect is present. If a boson is about to undergo an event with a certain probability $P$, and will end up after the event in a certain state $S$, then the actual probability of the boson undergolng this event is enhanced by the number of bosuns already in the final state $S$. If this number is $N$, then the actual probability of the event becomes

$$
P^{\prime}=P(1+N)
$$

Consider a photon about to undergo a scattering event. Tht photon has initial frequency $v$ and direction vector $\Omega$. It undergoes a scattering event and emerges with a final frequency $v^{\prime}$ and direction vector $\underline{\Omega}^{\prime}$. If the macroscopic differential scattering cross section in the absence of other photons is given by

$$
\sigma_{5}\left(v+v^{\prime}, \underline{\Omega} \cdot \underline{\Omega}^{\prime}\right)
$$


then in the presence of other photons we can write

$$
(1+N) \sigma^{2}\left(v+v^{\prime}, \underline{\Omega} \cdot \underline{\Omega}^{\prime}\right),
$$

where $\mathrm{N}$ is the occupation number of photons with frequency $v^{\prime}$ and direction vector $\Omega^{\prime}$.

The same effect occurs for the emission of photons. This is more commonly known as stimulated emission and is the primary process involved in laser physics.(39)

The primary aim of this thesis is to address the role of induced Compton and inverse Compton scattering on the evolution of the photon energy spectrum for a variety of initial photon distributions. One dimensional spatial dependence is also included. Although this dependence is suppressed in many instances to address induced scattering problems, several spatially dependent problems are included to demonstrate the ability of the codes developed to handle problems of this type.

An examination of the evolution of the radiation frequency profile with and without induced scattering effects is explored in this thesis. Also, the consequent change in electron energy density with and without the inclusion of induced scattering is addressed. These problems yield information on the importance of induced Compton and inverse Compton scattering in radiation transport problems. 
1.2 Review of Prior and Related Work

\subsubsection{Linear Radiation Transport}

One technique currently being investigated and used to solve radiation transport problems is Monte Carlo, $(4,40)$ This method, however, cannot alone calculate the effects caused by induced processes. In order to calculate induced effects, the number of photons in the final state must already be known. This information is unavalable in a Monte Carlo approach. One possible method of calculating these effects using Monte Carlo would involve a combination of Monte Carlo techniques and the method of successive approximations. The approach could be the following. First, a Yonte Carlo calculation is done for the problem in question which does not include induced effects. This yields an initial solution for the specific Intensity. A second Monte Carlo calculation can now be run on the same problem with the inclusion of induced effects. The new probabilities can be adjusted for the inclusion of induced effects using the distribution found in the initlal run. This gives an improved estimate of the specific intensity. This process can be repeated using successively more accurate solutions until convergence to the desired accuracy is obtained.

There are several drawbacks to the approach just discussed. In order to obtain reasonably accurate results, a large number of photons would have to be used for good statistics. Time considerations when doing computer simulations would limit the accuracy available. Also, the fact that several Monte Carlo runs are required for convergence to the desired solution would result in the 
use of prohibttively large amounts of computer time. This makes a solution of this type too expensive to be practical at this time. Hence, another approach must be taken.

The complete radiation transport equation, as is shown in Chapter Two, is both integro-differential and nonlinear. Because of the difficulty in solving the complete radiation transport equation, approximations must be made to obtain numerical results with currently available techniques. Discussed in this section are some of those techniques which address the linear form of the transport equation and, hence, do not include induced scattering effects.

One of the most commonly used methods of approximating the radiation transport equation is the variable Eddington diffusion model. $(2,3,7)$ This approximation, which is discussed more fully in Chapter Two, has several drawbacks. As a diftusion model, it is limited as to the amount of directional information which can be obtained. The variable Eddington method contains potentially mort accurate directional information then standard diffusion theory.(9) This is due to the method of closure used. However, a semi-empirical formula is needed to complete the approximation. This relation, which varies according to the problem being addressed, is not readily available. Modeling of this relation has been attempted by Minerbo( 8$)$ and Pomraning(41). Most important in the context of this thesis, this method does not contain any information concerning Induced scattering effects. 
Another method that can be used to help simplify the transport equation is the $P_{N}$ approximation. (9) This method is used to represent the angular dependence in the transport equation. Briefly stated, the $P_{N}$ method involves the expansion of both the specific intensity and the scattering kernel in an infinite series using Legendre polynomials as a basis. If azimuthal symmetry is assumed, these expansions can be written as

$$
I(\underline{r}, v, \underline{\Omega}, t)=\sum_{n=0}^{\infty} I_{n}(\underline{I}, v, t) P_{n}(u) \frac{(2 n+1)}{4 \pi}
$$

and

$$
\sigma_{s}\left(\underline{r}, v^{\prime}+v, \Omega^{\prime} \cdot \Omega\right)=\sum_{n=0}^{\infty} \frac{(2 n+1)}{4 \pi} \sigma_{s n}\left(\underline{r}, v^{\prime}+v\right) P_{n}\left(\underline{\Omega}^{\prime} \cdot \underline{\Omega}\right) .
$$

Substitution of these expressions into the transport equation and subsequent manipulation result in a set of equations to be solved simultaneously for the expansion coefflcients $I_{n}(\underline{r}, v, t)$. This series of equations is then truncated after $\mathrm{N}+1$ equations in terms of $\mathrm{N}+2$ dependent variables. A common way of closing this set of equaticns is accomplished by setting

$$
I_{N+1}(\underline{r}, v, t)=0
$$


This method was not found applied to the nonlinear transport equation in the literature. Hence, a complete derivation is included in Chapter Two. The $P_{N}$ method will be used in this thesis to represent the angular dependence of the specific intensity and the scattering kernel.

The frequency integrals still present in the transport equation must be approximated to solve the $P_{N}$ equations. The multigroup method $(9)$ is in common use to address this problem. In this technique, the photon energy spectrum is divided into G energy groups. Each of the $P_{N}$ equations can then be expanded into $G$ equations with the frequency integrals replaced by sums over the photon groups. This leaves $G(N+1)$ equations in $G(N+1)$ unknowns to be solved simultaneously. In this thesis, the frequency integrals are handled somewhat differently. Instead of averajing over frequency groups, the equations are solved at individual frequency points. All quantities are assumed linear between these points.

\subsubsection{Nonlinear Radiation Transport}

The treatment of nonlinear radiation transport has been addressed for problems of induced Compton scattering through several approaches now to be discussed.

A. S. Kompaneets has developed a partial differential equation formulation for a spatially homogeneous Compton scattering problem.(10) It was derived in the nonrelativistic approximation for Compton scattering in a Maxwellian distribution of electrons. Kompaneets kinetic equation can be written as 


$$
\frac{\partial n}{\partial y}=\frac{1}{x^{2}} \frac{\partial}{\partial x} x^{4}\left(\frac{\partial n}{\partial x}+n+n^{2}\right) .
$$

In this equation, $\mathrm{n}$ is the photon occupation number

$$
\mathbf{n}=\frac{c^{2} I}{2 h v^{3}}
$$

with

$$
\mathrm{x}=\frac{\mathrm{hv}}{\mathrm{kT}}
$$

and $\mathrm{y}$ is a dimensionless time,

$$
y=\left(m c^{2} / k T\right)(L / c)
$$

where $\mathrm{L}$ is the Compton mean free path and $\mathrm{N}_{\mathrm{e}}$ is the electron number density. (10)

$$
L=1 /\left[\mathrm{N}_{\mathrm{e}}(8 \pi / 3)\left(\mathrm{e}^{2} / \mathrm{mc^{2 }}\right)^{2}\right]
$$

It was found by Zel'dovich and Levich (11) that in the absence of absorption the photons undergo Bose condensation. Coste and Peyraud(12) and Zel'dovich, Levich, and Syundeav(13) found that, when scattering dominates, an initially narrow spectral line will shift downward in frequency space and build up at the lower spectral end of 
the initlal profile. Other work by Montes (14) and Galeev and Syunyaev(15) on the nonlinear transport equation has yielded what appears to be photon solitons moving downward in frequency space with narrow initial spectra.

The work that has bien done thus far on the nonlinear radiation transport equation is very limited in nature. Kompaneets equation, for example, is extremely difflcult to solve without introducing several approximations. Most common is the assumption that $n \gg l$, and, hence, $n^{2}>>n$. This allows removal of the linear scattering term. This same assumption is made in the literature for other approaches to this problem. $(14,15)$ Inclusion of spatial dependence as well as induced scattering effects has not been addressed in the literature.

This thesis addresses problems where induced scattering is important and linear scattering is also important. The scattering medium is an electron gas. Hence, no absorption can occur.

\subsection{Summary of Thesis}

\subsubsection{General Methods}

The radiation transport equation, discussed more fully in Chapter Two, is an integro-differential equation quite similar to the transport equation used for neutron transport problems.(42) It represents a photon balance of source terms and sink terms. However, unlike the neutron transport equation, the inclusion of induced effects results in a nonlinear equation. Without induced scattering, the radiation transport equation is linear. This nonlinearicy 
appears in both the inscattering and outscattering terms of the equation. These terms become quadratic in the dependent variable $I(\underline{r}, v, \underline{\Omega}, t)$, the specific intensity. This occurs because the occupation number $\mathrm{N}$ is proportional to $\mathrm{I}(\underline{r}, v, \underline{s}, \mathrm{t}),(6)$

The radiation transport equation can be written as

$$
\begin{aligned}
& \frac{1}{c} \frac{\partial I}{\partial t}(\underline{r}, v, \underline{\Omega}, t)+\underline{\Omega} \cdot \nabla I=S(\underline{r}, v, \underline{\Omega}, t)(1+N) \cdots \sigma_{a}(\underline{r}, v) I \\
& +\int_{0}^{\infty} d v^{\prime} \int_{4 \pi} d \Omega^{\prime} \frac{v}{v^{\prime}} \sigma_{S}\left(\underline{r}, v^{\prime}+v, \Omega^{\prime} \cdot \underline{\Omega}, t\right) I\left(\underline{r}, v^{\prime}, \underline{S_{0}^{\prime}}, t\right)(1+\underline{t}) \\
& -\int_{0}^{\infty} d v^{\prime} \int_{i \pi} d \Omega^{\prime} \sigma_{s}\left(\underline{r}, v+v^{\prime}, \underline{\Omega} \cdot \underline{\Omega}^{\prime}\right) I(\underline{r}, v, \underline{\Omega}, t)\left(1+\underline{N}^{\prime}\right) .
\end{aligned}
$$

In this equation, 1 is the specific intensicy, $S$ is a generd soulce term, $\sigma_{a}$ is the macroscopic absorption cross section, and $\sigma_{b}$ is the macroscopic scattering kernel. Note the tactor ( $1+: i)$ introduced to include both induced emission and induced scatcering. Since $N \times I$, it can be seen that the scattering terms become quadratic in 1 .

This thesis is concerned with approximate solutions to Eq. (9). Problems which have spherical syametry will be considered. Tris simplifies Eq. (9) considerably.

Several approximations are made in order to reduce Eq. (9) to a more manageable form. Representation of the angular dependence is accomplished ty utilization of the $P_{N}$ approximation. This thesis solves problems in which the $P_{1}$ approximation is used. A brief outline of the application of the techniques used in this thesis fur 
solution of the $\mathrm{P}_{3}$ equations is included. However, actual solution of the $P_{3}$ equations is outside the scope of this thesis.

The $P_{i}$ and $P_{3}$ equations form a closed set of equations which, as will be discussed in Chapter Three, are hyperbolic in nature. The $\mathrm{P}_{2}$ equations are mixed equations with a parabolic nature as well. The hyperbolic equations allow for -zcurate treatment of discontinuous solutions which can occur in some situations. An example of this is given in Chapter Four.

Finite differencing techniques $(43)$ are difficult to use on the nonlinear radiation transport equation. The quadratic nonlinearity makes simultaneous solution of the finite difference equations extremely difficult. Since the $P_{1}$ and $P_{3}$ equations to be addressed are hyperbolic, the method of characteristics can be considered.(16) In this approach, the ${ }_{P_{N}}$ equations are first reduced to canonical form. Then, the solutions in terms of new canonical variables are found at intersection paists of the characteristic curves.

These techniques, although representing the angular dependence, do not address the frequency integrals still present in the equations. This is done by solving for the intensity at specific frequency points. All quantities are assumed linear between these points. The frequency integrals are evaluated using the trapezoid rule for numerical integration. Choice of these points and more detalled information on the techniques for evaluating the frequency integrals are discussed in Chapter Four. 
The above methods are used in conjunction with the method of successive approximations to obtain the results of this thesis.

\subsubsection{Physical Situations Addressed}

This thesis addresses problems in which the dominant interactions are Compton and inverse Compton scattering. Problems addressed are those in which a radiation field is introduced into an electron field. Modeling of the movement of the radiation through the electron field is accomplished. Also of interest is the evolution of the radiation frequency profile upon inceraction with the electrons.

The scattering medium is modeled using a relativistic Maxwellian distribution of electrons. Since the scattering medium used in this thesis is an electron gas, no absorption or emission was included. Inclusion of these effects and solution for different types of media can be accomplished using the same techniques outlined in this thesis for the Compton scattering problems. What is needed for these types of problems is detailed information concerning the absorption and emission probabilities. These effects can be added to the codes developed here without difficulty due to the nature of the method of characteristics. This process will be discussed in Chapters Three and Four.

A number of physically interesting situations was studied for this thesis. Problems with blackbody initial spectra are solved. Also, problems with initially sharp spectral features represented by step functions in frequency space are addressed. In all cases, the 
codes developed were designed to handle problems in spherical geometry with radial spatial dependence and temporal dependence.

The scattering kernel viewed in the electron rest frame is modeled using the Klein-Nishina formula for Compton scattering.(17) It is necessary to Lorentz transform the scattering kernel and average over the electron velocity distribution in order to solve the problems addressed. This process has been well documented in the Iiterature. $(18,19,20)$ Tables of these cross sections for various entrance and exit frequencies have been compiled by Matteson, Pomraning, and Wilson.(21) A code which duplicates these results has already been developed at the Los Alamos National Laboratory by Gerald Minerbo.(44) The code written by Minerbo has been adapted for use as a subroutine in the calculations done for this thesis. A comparison of the results of Minerbo's code to the cross sections tabulated in Ref. (21) shows close agreement at all of the energies and frequencies checked. Minerbo's code is adapted as the subroutine SECTION in the code listing given in Appendix III.

Several examples of spatially dependent problems are given in Chapter Four. One demonstrates the ability of the codes to handle discontinuous problems. A plot of the isotropic intensity versus radius is shown. A second example contains all effects considered. This example contains one-dimensional spatial dependence, temporal dependence, and both linear and nonlinear scattering. A plot of the radiation energy density versus radius is shown. In this problem, Isotropic radiation is introduced in a finite area centered at the origin and allowed to move outward through the electron field. 
other problems addressed in this thesis can be divided into tho basic categorfes. These were Intended to examine the evolution of the frequency profile with time. Problems whose initial distribution is that of a blackbody spectrum and situations in which sharp spectral features are present initially are solved. These problems are solved when the electron temperature is held constant and, also, when the electron temperature is allowed to vary as a result of interaction with the radiation field.

Two types of problems are examined in the problems with an initial blackbody photon spectrum. One begins with the phuton temperature much greater than the electron temperature. The otner has the photon temperature much less than the electron temperatur= initially.

Problems with several sharp spectral features are also examined. Step sunctions in frequency space are used to model these features. These problems show a more marked difference when induced scattering is included than did those in which the initial spectrum was that of a blackbody.

In addition to examining the photon energy spectrum, the effect of induced scattering on the rate of energy exchange between the photon and electron fields is of interest. This energy exchange rate is evaluated by requiring conservation of energy.

One of the crucial assumptions used in this thesis is that the electron distribution remains well-described by a relativistic Maxwellian distribution. It is assumed that, as the electrons interact with the radiation field, the electron relaxation time is 
sufficiently short to maintain its Maxwellian character. This will be discussed more fully in Section 3.3 .

The numerical work done in this thesis was done on the computer facilities at the Los Alamos National Laboratory. CDC-7600 and CRAY-1 computers were used for all of the codes. These codes are written in Fortran. The codes which were developed to solve the problems posed in this thesis are all variants of the same code. These codes were not intended for use in large scale simulation codes where radiation tcansport treatment is required. However, adaptation could be accomplished. Streamlining would also be required if computer time is a serious constraint. Ir is believed that the computing time could be reduced by as much as a factor of two. 


\section{RADIATION TRANSPORT EQUATIONS AND THEIR APPROXIMHTIUIS}

\subsection{The Linear Radiation Iransport Equation}

This section concalns a brief discussicn of the photon transport equation. For details of several approaches to the derivation of tile transport equation, the reader is referred to Ref. (9). The radiation iransport equation is a phase space balance equation. It states that the number of photons which enters or leaves a differential solid angle $d \Omega$ about $\Omega$, differential frequency interval ov about $v$, and differential volume eiement dy about $I$ is equal to the sources minus the sink terms. Photons car strean in or out, scatter in or out, or be emitted or absorbed in a differencial volune element of phase space.

The photon angular number density is defined as

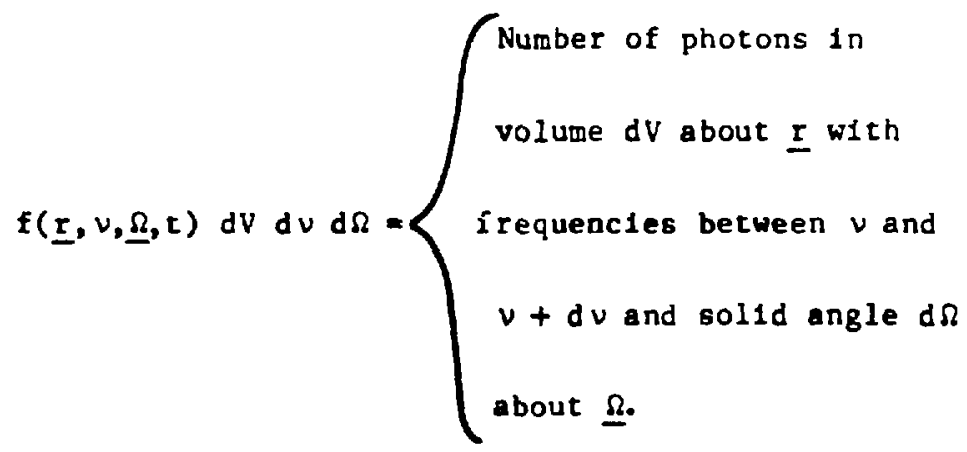


Writing a photon balance equation in terms of the angular number density yields the expression,

$$
\begin{aligned}
& \frac{\partial f}{\partial t}(\underline{r}, v, \underline{\Omega}, t)+c \underline{\Omega} \cdot \nabla f=q(\underline{r}, v, t)-c \sigma_{a}(\underline{r}, v) f \\
& +c \int_{0}^{\infty} d v^{\prime} \int_{4 \pi} d \Omega^{\prime}\left[\sigma_{s}\left(\underline{r}, v^{\prime} \cdot v, \underline{\Omega}^{\prime} \cdot \underline{\Omega}\right) f\left(\underline{r}, v^{\prime}, \underline{\Omega^{\prime}}, t\right)-\right. \\
& \left.\sigma_{s}\left(\underline{r}, v \rightarrow v^{\prime}, \underline{\Omega} \cdot \underline{\Omega}^{\prime}\right) f(\underline{r}, v, \underline{\Omega}, t)\right] .
\end{aligned}
$$

Tnis balance equation does not as yet contain the effects due to induced processes. These effects will be added in Section 2.2.

The two terms on the left hand side of the photon balance equation represent the cotal change in the number of photons with time. It should be noted that the substantial time derivative is

$$
\frac{d}{d t}=\frac{\partial}{\partial t}+\underline{v} \cdot \nabla
$$

where for photons,

$$
\underline{v}=\underline{c} \underline{\Omega}
$$

The term $q(\underline{r}, v, t)$ is a volumetric source term. It will be assumed isotropic. However, it need not necessarily be isotropic. The source will be allowed to have both spatial and temporal dependence. This source will be retalned in the general form above for derivation, although actual problems solved will not include 
absorption or emission. This is because the transport medium to be considered is an electron gas.

The second term on the right hand side of the balance equation (11) represents a sink due to absorption. Here $o_{a}(r, v)$ is the macroscopic absorption cross section which can be spatially dependent. Inclusion of absorption and emission in the codes developed to solve the problems of interest in this thesis will be discussed in Chapters Three and Four. The last two terms in Eq . (11) represent photon scattering. The first expression in brackets is the inscattering term. The second expression represents outscattering. In this thesis, scattering will occur due to photon interaction with free electrons. Compton scattering will be the primary process involved.

The scattering kernel $\sigma_{s}\left(\underline{r}, \nu \rightarrow \nu^{\prime}, \underline{s} \cdot \cdot \underline{s}\right)$ is the macroscopic scattering cross section. In problems in which the electrons are in motion, the scattering kernel is averaged over the velocity distribution of the electrons.

Equation (11) can be rewritten in terms of the specific intensity $I(\underline{\mathbf{r}}, v, \underline{\Omega}, t)$ defined by

$$
I(\underline{r}, v, \underline{\Omega}, t)=\operatorname{chv} f(\underline{r}, v, \underline{\Omega}, t) .
$$

Substitution of Eq. (14) into Eq. (11) yields the expression

$$
\frac{1}{c} \frac{\partial I}{\partial t}+\underline{\Omega} \cdot \nabla I=S(\underline{r}, \nu)+\sigma_{a}(\underline{r}, \nu) I
$$




$$
\begin{gathered}
+\int_{0}^{\infty} \mathrm{d} v^{\prime} \int_{4 \pi} \mathrm{d} \Omega^{\prime}\left[\frac{v}{v^{\prime}} \sigma_{s}\left(\underline{r}, v^{\prime} \rightarrow v, \underline{\Omega^{\prime}} \cdot \underline{\Omega}\right) I\left(\underline{r}, v^{\prime}, \underline{\Omega} \underline{\Omega}^{\prime}, \mathrm{r}\right)\right. \\
\left.-\sigma_{s}\left(\underline{r}, v \rightarrow v^{\prime}, \underline{\Omega} \cdot \underline{\Omega}^{\prime}\right) I(\underline{r}, v, \underline{\Omega}, \mathrm{t})\right],
\end{gathered}
$$

where

$$
S(\underline{r}, v)=h v q(\underline{r}, v) .
$$

The total scattering cross section is given by

$$
\sigma_{s}(\underline{r}, v)=\int_{0}^{\infty} d v^{\prime} \int_{4 \pi} d \Omega^{\prime} \sigma_{s}\left(\underline{r}, v+v^{\prime}, \underline{\Omega} \cdot \underline{\Omega}^{\prime}\right) .
$$

Equation (15) can be rewritten as

$$
\begin{aligned}
& \frac{1}{c} \frac{\partial I}{\partial t}+\underline{\Omega} \cdot \nabla I(v, \underline{\Omega})=S(v)-\sigma_{t}(v) I \\
& +\int_{0}^{\infty} d v^{\prime} \int_{4 \pi} d \Omega^{\prime} \frac{v}{v^{\prime}} \sigma_{s}\left(v^{\prime}+v, \Omega^{\prime} \cdot \Omega\right) I\left(v^{\prime}, \Omega^{\prime}\right),
\end{aligned}
$$

where the spatial and temporal arguments have been suppressed for ease of writing, and,

$$
\sigma_{t}(v)=\sigma_{a}(v)+\sigma_{s}(v)
$$

is the total macroscopic cross section.

Equation (18) 1s the most common form of the radiation transport equation in the absence of induced effects. It is a linear 
integro-differential equation quite similar to the neutron transport equation commonly used in reactor physics problems. (42)

\subsection{The Nonlinear Radiation Transport Equation}

In order to formulate a more accurate physical model, induced effects must be included in the radiation transport equation. The effects of induced emission and indused scattering are quite easy co include. However, the resulting transport equation is nonlinear and difficult to soive. Since photons are bosons, the probability of an event, such as emission and scattering, must be altered when a radiation field is present. If the photon occupation number of the final state of an event is given by $N$, and if the probability of tnis event occurring is given by $P$ in the absence of a radiation field, then the probability of the event occurring in the presence of other radiation is enhanced and becomes $(6)$

$$
P^{*}=P(1+i)
$$

For photans, the occupation number can be derived in the following manner. The photon occupation number $N$ can be writcen as,

$$
N=\int d V \int_{\Delta} d \nu \int d \Omega f(\underline{r}, \nu, \underline{\Omega}, t)
$$

A differential volume element in momentum space can be written as

$$
d^{3} p=p^{2} d p d \Omega
$$


where

$$
p=\frac{h v}{c} \text {. }
$$

This implies that

$$
d^{3} p=(h / c)^{3} v^{2} d v d a
$$

Since

$$
I(\underline{r}, v, \underline{\underline{n}}, t)=\operatorname{ch} v f(\underline{r}, v, \underline{\Omega}, t),
$$

we have

$$
\therefore=\frac{c^{2}}{h^{4}} \int d v \int_{\Delta} d^{3} p I\left(\underline{r}, v, \underline{s, r}, \frac{1}{v^{3}} .\right.
$$

The volume $\Delta$ is given by $(6)$

$$
\Delta=\Delta \underline{\underline{r}} \Delta \underline{\mathrm{p}}=\frac{\mathrm{h}^{3}}{2} .
$$

Hence, we can write

$$
N=\frac{c^{2}}{v^{3} h^{4}} I(\underline{r}, v, \underline{\Omega}, t) \Delta
$$


or

$$
N=\frac{c^{2}}{2 h \nu^{3}} I(\underline{r}, \nu, \underline{\Omega}, t)
$$

Therefore, we find that the probability of the event for photons is glven by

$$
\mathbf{P}^{*}=P\left[1+\frac{c^{2}}{2 h \nu^{3}} I(\underline{r}, \nu, \underline{\Omega}, t)\right] .
$$

If Induced effects are included in the transport equation, Eq. (15) must be rewritien as

$$
\begin{aligned}
& \frac{1}{c} \frac{\partial I}{\partial t}(v, \underline{\Omega})+\underline{\Omega} \cdot \nabla I(v, \underline{\Omega})=S(v)(1+N)-\sigma_{a}(v) I(v, \underline{\Omega}) \\
& +\int_{0}^{\infty} d v^{\prime} \int_{4 \pi} d \Omega^{\prime} \frac{v}{v^{\prime}} \sigma_{s}\left(v^{\prime} \rightarrow v, \underline{\Omega}^{\prime} \cdot \Omega\right) I\left(v^{\circ}, \underline{\Omega}^{\prime}\right)(1+N) \\
& -\int_{0}^{\infty} d v^{\prime} \int_{4 \pi} d \Omega^{\prime} v_{s}\left(v+v^{\prime}, \underline{\Omega} \cdot \Omega^{\prime}\right) I(v, \underline{\Omega})\left(1+N^{\prime}\right),
\end{aligned}
$$

where

$$
N^{\prime}=\frac{c^{2}}{2 h v^{\prime 3}} I\left(v^{\prime}, \Omega^{\prime}\right)
$$

Subst1tuting Eq. (29) Into Eq. (31) yields the expression 


$$
\begin{aligned}
& \frac{1}{c} \frac{\partial I}{\partial t}(\nu, \Omega)+\Omega \cdot \nabla I(\nu, \Omega)=s(\nu)\left[1+\frac{c^{2}}{2 h \nu^{3}} I(\nu, \Omega)\right]-\sigma_{a}(\nu) I(\nu, \Omega) \\
& +\int_{0}^{\infty} d v^{\prime} \int_{4 \pi} d \Omega^{\prime}-\frac{\nu}{v^{\prime}} \sigma_{s}\left(\nu^{\prime} \rightarrow \nu, \Omega^{\prime}-\Omega\right) I\left(\nu^{\prime}, \Omega^{\prime}\right)\left[1+\frac{c^{2}}{2 h v^{3}} I(\nu, \Omega)\right] \\
& -\int_{0}^{\infty} d v^{\prime} \int_{4 \pi} d \Omega^{\prime} \sigma_{s}\left(v+v^{\prime}, \Omega \cdot \Omega^{\prime}\right) I(v, \Omega)\left(1+\frac{c^{2}}{2 h v^{\prime 3}} I\left(v^{\prime}, \Omega^{\prime}\right)\right] .
\end{aligned}
$$

It can be seen that the radiation transport equation with induced effects included is no longer linear. It now contains several quadratic nonlinearities in the scattering terms.

We now introduce the following definitions;

$$
S(\nu)=\sigma_{a}^{\prime}(\nu) B(\nu)
$$

and

$$
\sigma_{a}(v)=\sigma_{a}^{\prime}(v)\left[1+\frac{c^{2}}{2 h v^{3}} B(v)\right] .
$$

Entering Eqs. (34) and (35) into Eq. (33) produces

$$
\begin{aligned}
& \frac{1}{c} \frac{\partial I}{\partial t}+\Omega \cdot \nabla I=\sigma_{a}^{\prime}(v)\left[B(v)-I\left(v, \Omega^{\prime}\right)\right] \\
& +\int_{0}^{\infty} d v^{\prime} \int_{4 \pi} d \Omega^{\prime} \frac{v}{v^{\prime}} \sigma_{s}\left(v^{\prime}+v, \Omega^{\prime} \cdot \Omega\right) I\left(v^{\prime}, \Omega^{\prime}\right)\left[1+\frac{c^{2}}{2 h v^{3}} I(v, \Omega)\right] \\
& -\int_{0}^{\infty} d v^{\prime} \int_{4 \pi} d \Omega^{\prime} \sigma_{s}\left(v+v^{\prime}, \underline{\Omega} \cdot \Omega^{\prime}\right)
\end{aligned}
$$




$$
\left.\times I(v, \Omega) ! 1+\frac{c^{2}}{2 h v^{\cdot 3}} I\left(v^{\prime}, \Omega^{\prime}\right)\right)
$$

If we consider the case of spherical georetry bith azinutial symetry, the transport equation becones,

$$
\begin{aligned}
& \frac{1}{c} \frac{\partial I}{\partial t}(\nu, \mu)+\frac{\partial I}{\partial r}(\nu, \mu)+\frac{\left(1-\mu^{2}\right)}{r} \frac{\partial I}{\partial j}(\nu, \mu)=c_{a}^{\prime}(\nu) \mid \partial(\nu, I(\mu, \mu)
\end{aligned}
$$

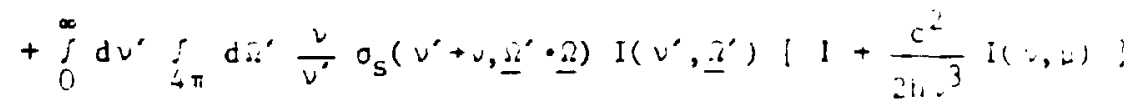

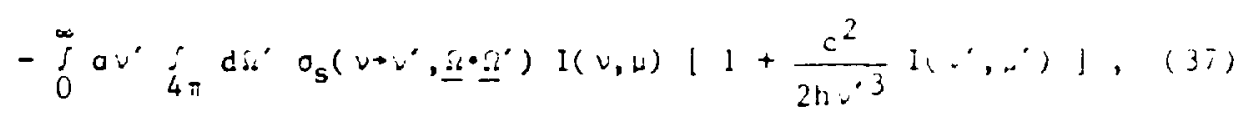

where $\mu=\cos \theta$.

Ints norlinear fork of the radiation transpori equation bitu induced effects provides the basis for the research done in tuls thesis.

Several comments should be wade at this point with regards io boundary and initial condizions needed for solution of Eq. (37). One of the more common boundary conditions of interest in radiation transport problems is that of a non-reentrant surface. (9) This can be expressed as

$$
I\left(\underline{r}_{s}, v, \underline{\Omega}, t\right)=\Gamma\left(\underline{r}_{s}, v, \underline{\Omega}, t\right)
$$

for $\underline{n} \cdot \underline{\Omega}<0$, where $\Gamma=0$ for a non-reentrant surface, and $\Gamma\left(\underline{r}_{s}, v, \underline{\Omega}, t\right)$ can be a specifled function fer a known incoming flux of radiation for 
other problems. In this case ss $_{\text {is }}$ a point on the boundary surface and $n$ is an outward unit vector normal to the boundary surface at the point $\underline{r}_{s}$

A general initial condition can be written as

$$
I(\underline{r}, \nu, \underline{\delta}, 0)=\Lambda(\underline{r}, \nu, \underline{\Omega}),
$$

where $\Lambda(\underline{\mathbf{r}}, v, \underline{\underline{2}})$ is a known initial distribution.

Although obtaining solutions to the radiation transport equation with the inclusion of induced effects is extremely difficult, the solution for the case of complete thermodynamic equilibrium is well known. $(9,32,33)$ Inis solution is known as the Planck blackbody distribution $B_{D b}(v)$, where

$$
B_{b b}(v)=\frac{2 h v^{3}}{c^{2}}\left(e^{(h v) /(k T)}-1\right)^{-1} .
$$

In the case where local thermodynamic equilibrium can be assumed, the source function $B(v)$ in Eq. (37) is given by Eq. (40). Also, the modified absorption cross section $\sigma_{a}^{\prime}(v)$ becomes $(9)$

$$
\sigma_{a}^{\prime}(v)=\sigma_{a}(v)\left(1-e^{(h v) /(k I,)}\right.
$$

A useful relation in radiation transport problems is called the detalled balance condition. If we assume that scattering occurs through photon-electron interactions and that the electrons are in 
equilibrium, we are able to derive the detailed balance condition. Consider a steady state, spatially uniform problen. Eq.(37) can be written as

$$
\begin{aligned}
& 0=\sigma_{a}^{\prime}(\nu)[B(\nu)-I(\nu, \mu)] \\
& +\int_{0}^{\infty} d v^{\prime} \int_{4 \pi} d \Omega^{\prime} \frac{\nu}{v^{\prime}} \sigma_{s}\left(\nu^{\prime}+\nu, \Omega^{\prime}-\Omega\right) I\left(\nu^{\prime}, \nu^{\prime}\right)\left[1+\frac{c^{2}}{2 h \nu^{3}} I(\nu, \nu)\right] \\
& -\int_{0}^{\infty} d v^{\prime} \int_{4 \pi} d \Omega^{\prime} \sigma_{s}\left(\nu+\nu^{\prime}, \underline{\Omega} \cdot \Omega^{\prime}\right) I(\nu, \nu)\left[1+\frac{c^{2}}{2 h v^{\prime}} I\left(\nu^{\prime}, \nu^{\prime}\right)\right] .
\end{aligned}
$$

However, the solution to this problem is known to be given by Eq. (40). Hence, Eq. (42) can be written as

$$
\begin{aligned}
& \int_{0}^{\infty} d v^{\prime} \int_{4 \pi} d \Omega^{\prime} \frac{v}{v^{\prime}} \sigma_{s}\left(v^{\prime}+v, \Omega^{\prime} \cdot \underline{\Omega}\right) B_{b b}\left(v^{\prime}\right)\left[1+\frac{c^{2}}{2 h v^{3}} B_{b b}(\nu)\right] \\
& =\int_{0}^{\infty} d v^{\prime} \int_{4 \pi} d \Omega^{\prime} \sigma_{s}\left(v+v^{\prime}, \Omega \cdot \Omega^{\prime}\right) B_{b b}(v)\left[1+\frac{c^{2}}{2 h v^{\prime 3}} B_{b b}\left(v^{\prime}\right)\right] .
\end{aligned}
$$

In complete thermodynamic equilibrium, the number of photons scattered out of a particular state must equal the number of photons scattered into that state. Hence, we can rewrite Eq. (43) and obtain

$$
\begin{aligned}
& \frac{v}{v^{\prime}} \sigma_{s}\left(v^{\prime}+\nu, \Omega^{\prime} \cdot \Omega\right) B_{b b}\left(v^{\prime}\right)\left[1+\frac{c^{2}}{2 h v^{3}} B_{b b}(v)\right] \\
& =\sigma_{s}\left(v+v^{\prime}, \underline{\Omega} \cdot \Omega^{\prime}\right) B_{b b}(v)\left[1+\frac{c^{2}}{2 h v^{\prime 3}} B_{b b}\left(v^{\prime}\right)\right] .
\end{aligned}
$$


If we now use the expression for $B_{b b}(\nu)$ given by Eq. (40), we can obtain the detailed balance condition, namely,

$$
J_{s}\left(\nu \rightarrow v^{\prime}, \underline{\Omega} \cdot \underline{\Omega}^{\prime}\right)=\frac{v^{2}}{v^{\prime 2}} \mathrm{e}^{\frac{h\left(\nu-v^{\prime}\right)}{\mathrm{kT}}} \sigma_{\mathrm{s}}\left(v^{\prime} \rightarrow v, \underline{\Omega^{\prime}} \cdot \underline{\Omega}\right) .
$$

The blackbody solution is only valid when induced effects are included in the transport equation. If induced effects are not included, the solution to the same problem turns out to be the Wien distribution. (9)

It should be noted that the detailed balance condition is only applicable when the electrons are in local thermodynamic equilibrium. This will be assumed correct for all problems addressed in this thesis.

Another way to look at the detailed balance condition is the following. It is simply a statement that the number of photons which scatter from $d v d \Omega$ about $\nu$ and $\Omega$ into $d v^{\prime} d \Omega^{\prime}$ about $\nu^{\prime}$ and $\Omega^{\prime}$ is equal to the number of photons that scatter from $\mathrm{d} \nu^{\prime} \mathrm{d} \Omega^{\prime}$ about $\nu^{\prime}$ and $\Omega^{\prime}$ into duds about $v$ and $\Omega$ as must be the case in equilibrium. Hence, we can 1mmediately write ES. (44).

Several physical quantities of interest in radiation transport problems are defined as follows. The radiation energy density can be written as

$$
u(\underline{r}, t)=\frac{1}{c} \int_{0}^{\infty} d v \int_{4 \pi} d \Omega I(\underline{r}, v, \underline{\Omega}, t) .
$$


For the case of a Plancklan distribution, this becomes

$$
u=\frac{1}{c} \int_{0}^{\infty} d \nu \int_{4 \pi} d \Omega h \nu \frac{2 h v^{3}}{c^{2}}\left(e^{(h v) /(k T)}-1\right)^{-1}
$$

or

$$
\mathrm{u}=\mathrm{aT} \mathrm{T}^{4},
$$

where

$$
a=\frac{8 \pi^{5} k^{4}}{15 h^{3} c^{3}}
$$

This is also writter as

$$
u=\frac{40}{c} T^{4}
$$

where

$$
\sigma=\frac{a c}{4}
$$

is the Stephan-Boltzman constant. The radiation flux vector $\underline{F}(\underline{r}, t)$ can be written in terms of the specific intensity as 


$$
\underline{F}(\underline{r}, t)=\int_{0}^{\infty} d v \int_{4 \pi} d \Omega \underline{\Omega} I(\underline{r}, v, \underline{\Omega}, t) .
$$

Finally, the second moment of the specific intensity gives the radiation pressure tensor $\stackrel{P}{=}(\underline{r}, t)$ which describes the rate of flow of gomentum across a unit surface, namely,

$$
\underline{\underline{P}}(\underline{r}, t)=\frac{1}{c} \int_{0}^{\infty} d v \int_{4 \pi} d \Omega \underline{\Omega \Omega} I(\underline{r}, v, \underline{\Omega}, \tau) \text {. }
$$

It can be shown that the trace of the iressure tensor, (9)

$$
P_{x x}+P_{y y}+P_{z z}=u(\underline{r}, t),
$$

is the radiation energy density.

Since this thesis is concerned with radiation transport in an electron field, it 1s important to note the form taken by the scattering kernel. The primary process involved in the problems addressed in this thesis is Compton scattering. In problems in which the photon energy is low, such that h $\mathrm{Km}_{\mathrm{e}} \mathrm{c}^{2}$. Thompson scattering occurs.

The scattering kernel for Compton scattering is given by the Klein-Nishina formula, $(17,26)$ namely,

$$
\begin{gathered}
\sigma_{K N}\left(\nu+\nu^{\prime}, \underline{\Omega} \cdot \underline{\Omega^{\prime}}\right)=\frac{r_{0}^{2} N_{e}}{2 \gamma \nu}\left[1+\mu^{2}+\gamma \gamma^{\prime}(1-\mu)^{2}\right] \\
\quad \times \delta\left(\mu-1+\frac{1}{\gamma^{\prime}}-\frac{1}{\gamma}\right),
\end{gathered}
$$


where

$$
\mu=\Omega \cdot \Omega^{\prime}
$$

and

$$
\gamma=\frac{h v}{m_{e} c^{2}} .
$$

Also, $\mathrm{N}_{\mathrm{e}}$ is the electron number density, and $r_{0}$ is the classical electron radius given by

$$
r_{0}=\frac{e^{2}}{m_{e} c^{2}}
$$

The total scattering cross section can be found from Eq. to be (23)

$$
\begin{gathered}
\sigma_{S}(\nu)=2 \pi r_{0}^{2}\left\{\frac{(1+\gamma)}{\gamma^{3}}\left[\frac{2 \gamma(1+\gamma)}{1+2 \gamma}-\log (1+2 \gamma)\right]\right. \\
\left.+\frac{1}{2 \gamma} \log (1+2 \gamma)-\frac{(1+3 \gamma)}{(1+2 \gamma)^{2}}\right\} .
\end{gathered}
$$

A brief outline of the development of the radiation transport equation has been given above. The origin of the Induced effects was shown, and the scattering formulae needed have been briefly 
discussed. In order to obtain actual solutions, the transport equation must first be reduced to a more tenable form through a series of approximations.

2.3 Approximations of the Radiation Transport Equation

The radiation transport equation is an extremely difficult equation to solve without some sort of simplification. A number of these approximations will be discussed in this section.

One method of representing the angular dependence of the transport equation is provided by taking angular noments. If the zeroch and $f i r s t$ anguiar moments of the transport equation are taken, and higher order moments of the specific intensity are assumed to be zero, the resulting two equations comprise a form of the radiation diffusion equations.(9) An improvement on this technique is to express the second angular moment as some function times the zeroth angular moment. This factor is called the variable Eddington factor, and this approach, the variable Eddington methid. This method is commonly used to model radiation transport problems in dense fusion plasmas. (7)

Another method that can be used to eliminate the angular dependence in the transport equation is the $\mathrm{P}_{\mathrm{N}} \operatorname{method.~In~this~}$ technique the specific intensity and the scattering kernel are both expanded in an infinite series using Legendre polynomials as a basis. The resulting equation is then operated on by

$$
\int d \mu^{\prime} P_{n}\left(\mu^{\prime}\right) \quad \text { for } n=0,1,2, \ldots
$$


which results in an infinite number of equations. This set of equations 15 truncated after $n=N$ to form $N+1$ equations in $i+2$ dependent variables. The $\mathrm{N}+2^{\text {th }}$ dependent variable is set equal to zero to reduce the number of dependent variables to N+l. This formis a closed set of equations which is independent of $\Omega$.

Another aspect of the transport equation which is of prime concern is the handling of the frequency integrals. A cormon technique for representing tnese integrals is the multigroup Det?od. This technique has been in use for many years in neutrunics calculations. In this approxlmation, the photons are divided into $G$ energy groups. The frequency integrals can then be replaced by a summation over all energy groups. This leaves $G(N+1)$ equations to be solved for the incensity of each energy group. This technicue is usually used in conjunction with other techniques such as the $P_{i}$ method.

other techniques, (9) such as discrete ordinates, are available to approximace the transport equation but will not be discussed in this thesis. Several of the above techniques have been used or moditled for use in this thesis and will be discussed in some detail In Section 2.3.2.

\subsubsection{Diffusion Theory}

First, consider the diffusion equations in the absence of induced scattering. This is the form most commonly used.(22) The transport equation to be used is given by $\mathrm{Eq} \cdot(18)$ as 


$$
\begin{aligned}
& \frac{1}{c} \frac{\partial I}{\partial t}(v, \underline{\Omega})+\underline{\Omega} \cdot \nabla I(v, \underline{\Omega})=S(v)-\sigma_{\tau}(v) I(v, \underline{\Omega}) \\
& +\int_{0}^{\infty} d v^{\prime} \int_{4 \pi} d \Omega^{\prime} \frac{v}{v^{\prime}} \sigma_{s}\left(v^{\prime}+v, \underline{\Omega}^{\prime} \cdot \underline{S l}\right) I\left(v^{\prime}, \underline{\Omega}^{\prime}\right) .
\end{aligned}
$$

The following definitions are needed to derive the diffusion equations. The energy density of the radiation field per unit frequency is

$$
E(v)=\frac{1}{c} \int_{4 \pi} d \Omega I(\nu, \Omega)
$$

The radiative flux vactor per unit frequency is

$$
\underline{F}(v)=\int_{4 \pi} d \Omega I(v, \Omega) \underline{\Omega}
$$

Finally, the radiative pressure tensor per unit frequency is

$$
\stackrel{P}{=}(v)=\frac{1}{c} \int_{4 \pi} d \Omega I(\nu, \underline{\Omega}) \underline{\Omega} \text {. }
$$

Here, the spatial and time dependence of the intensity I has been suppressed. If we assume $I(\nu, \Omega)=I(\nu, \mu)$, 1.e., azimuthal symmetry, and consider problems in one-dimensional geonetry, Eq. (60) reduces to

$$
\frac{1}{c} \frac{\partial I}{\partial t}(\nu, \mu)+\mu \frac{\partial I}{\partial r}(\nu, \mu)+\frac{1}{2}(\alpha-1) \frac{\left(1-\mu^{2}\right)}{r} \frac{\partial I}{\partial \mu}(\nu, \mu)
$$




$$
=S(\nu)-\sigma_{t}(\nu) I(\nu, \mu)+K(\nu, \mu) \text {, }
$$

wh.ere

$$
K(\nu, \nu)=\int_{0}^{\infty} d v^{\prime} \int_{4 \pi} d \Omega^{\prime} \frac{\nu}{v^{\prime}} \sigma_{s}\left(v^{\prime} \rightarrow v, \Omega^{\prime} \cdot \Omega\right) I\left(v^{\prime}, \mu^{\prime}\right) .
$$

In the case where only Thompson scattering is important, and hence, no frequency shift occurs upon scattering, the induced terms cancel and the above equation is exact. In Eq. (64), $\alpha=1$ for plane geometry, and $\alpha=3$ for spherical geometry. In this case, the radiation energy density per unit frequency, the $r$-component of the radiation flux vector per unit frequency, and the $r r-c o m p o n e n t$ of the radiation pressure tensor per unit frequency become

$$
\begin{aligned}
& E(r, v, t)=\frac{2 \pi}{c} \int_{-1}^{1} d \mu I(r, \nu, \mu, t), \\
& F(r, \nu, t)=2 \pi \int_{-1}^{1} d \mu I(r, \nu, \mu, t) \mu,
\end{aligned}
$$

and

$$
P(r, \nu, t)=\frac{2 \pi}{c} \int_{-1}^{1} d \mu I(r, \nu, \mu, t) \mu^{2}
$$

We now take the first two moments of Eq. (64) to obtain the diffusion equations, namely, 


$$
\frac{\partial E}{\partial t}+\frac{1}{r^{\alpha-1}} \frac{\partial}{\partial r}\left(r^{\alpha-1} F\right)+c \sigma_{t}(\nu) E=4 \pi S(\nu)+2 \pi \int_{-1}^{1} d \mu K(\nu, \mu)
$$

and

$$
\frac{1}{c} \frac{\partial F}{\partial t}+c\left[\frac{\partial P}{3 r}+\frac{a-1}{2 r}(3 P-E) 1+\sigma_{\tau}(\nu) F=2 \pi \int_{-1}^{1} d \mu \nu K(\nu, \mu) .\right.
$$

If Thompson scattering is assumed, the scattering kernel becomes(2)

$$
\sigma_{S}\left(v^{\prime}+v, \underline{\Omega}^{\prime} \cdot \Omega\right) \times \frac{1}{2}\left[1+\left(3 \mu^{2}-1\right)\left(3 \mu^{\prime 2}-1\right)\right]
$$

and there is no frequency change. In this case, Eqs. (70) and become

$$
\frac{\partial E}{\partial t}+\frac{1}{r^{\alpha-1}} \frac{\partial}{\partial r}\left(r^{\alpha-1} F\right)+c \sigma_{a}(\nu) E=4 \pi S(\nu)
$$

and

$$
\left.\frac{1}{c} \frac{\partial F}{\partial t}+c l \frac{\partial P}{\partial r}+\frac{(\alpha-1)}{2 r}(3 P-E)\right)+\sigma_{\tau}(\nu) F=0,
$$

where

$$
\sigma_{t}(v)=\sigma_{a}(v)+\sigma_{s}(v)
$$


Equations (73) and (74), however, do not form a closed set as we have two equations in three dependent variables, $E, F$, and $P$. If this set is closed by setting $P=0$, we arrive at the standard diffusion equations for radiative transport with an isotropic source.

Another method for closing the set of moment equations is called the variable Eddington method. In this method, the rr-component of the pressure tensor is replaced with the semi-empirical formula

$$
P(v)=f(r, v, t) E(v)
$$

The factor $f(r, v, t)$ is called the Eddington factor. This factor must be determined on the basis of the physical problem being addressed. Ideally, if $f(r, v, t)$ were known exactly, Eqs. (73) and (74) would be equivalent to the full transport equation when using the same assumptions utilized in the derivation of Eqs. (73) and (74). The principle requirement of $f(r, v, t)$ is that it reduces to a value of $1 / 3$ for oprically thick regions and to a value of 1 in optically thin regions. The Eddington factor has been explored at great length. And, this technique is used quite often. However, knowledge of the Eddington factor is quite limited. Also, the lack of induced effects In this technique make it unacceptable for the type of problem to be addressed in this thesis.

A more general way of writing the diffusion approximation, also known as the Eddington approximation, is to first assume that the specific intensity can be written as 


$$
I(\underline{r}, v, \underline{\Omega}, t)=\frac{1}{4 \pi} I_{0}(\underline{\underline{r}}, v, t)+\frac{3}{4 \pi} \underline{\Omega} \cdot \underline{I} \underline{I}_{l}(\underline{r}, v, t),
$$

where

$$
I_{0}(\underline{r}, \nu, t)=\int_{4 \pi} d \Omega I(\underline{r}, \nu, \underline{\Omega}, t)
$$

and

$$
\underline{I}_{l}(\underline{r}, v, r)=\int_{4 \pi} d \Omega \underline{\Omega} I(\underline{r}, v, \Omega, t) .
$$

Equation (77) is substituted into Eq. (36), and, the first two moments of the resulting equation are taken. This is done by operating on the equation with $\int_{4 \pi}$ di and $\int_{4 \pi \text {.2d }}$. If this is done, the following two equations result:

$$
\begin{aligned}
& \frac{1}{c} \frac{\partial I_{0}(v)}{\partial t}+\nabla \cdot I_{1}(v)=\sigma_{a}^{\prime}(v)\left[4 \pi B(v)-I_{0}(v)\right]-\sigma_{s}(v) I_{0}(v) \\
& +\int_{0}^{\infty} d v^{\prime} \frac{v}{v^{\prime}} \sigma_{s 0^{\prime}}\left(v^{\prime}+v\right) I_{0}\left(v^{\prime}\right)+\frac{c^{2}}{8 \pi h} I_{0}(v) \int_{0}^{\infty} d v^{\prime}\left[\frac{1}{v^{2} v^{\prime}} \sigma_{s 0}\left(v^{\prime}+v\right)\right. \\
& \left.-\frac{1}{v^{\prime 3}} \sigma_{s 0}\left(v+v^{\prime}\right)\right] I_{0}\left(v^{\prime}\right)+\frac{3 c^{2}}{8 \pi h} I_{I}(v) \cdot \int_{0}^{\infty} d v^{\prime}\left[\frac{1}{v^{2} v^{\prime}} \sigma_{s !}\left(v^{\prime}+v\right)\right. \\
& \left.-\frac{1}{v^{\prime 3}} \sigma_{s 1}\left(v+v^{\prime}\right)\right] I_{1}\left(v^{\prime}\right)
\end{aligned}
$$

and 


$$
\begin{aligned}
& \frac{1}{c} \frac{\partial \underline{I}_{1}}{\partial t}+\frac{1}{3} \nabla I_{0}+\left[\sigma_{a}^{\prime}+\sigma_{s}\right] \underline{I}_{1}=\int_{0}^{\infty} d v^{\prime} \frac{v}{v^{\prime}} \sigma_{s 1}\left(v^{\prime} \rightarrow v\right) \underline{I}_{l}\left(v^{\prime}\right) \\
& +\frac{c^{2}}{8 \pi h} I_{0} \int_{0}^{\infty} d v^{\prime}\left[\frac{1}{v^{2} v^{\prime}} \sigma_{s 1}\left(v^{\prime}+v\right)-\frac{1}{v^{\prime}} \sigma_{s l}\left(v+v^{\prime}\right)\right] \underline{I}_{1}\left(v^{\prime}\right) \\
& +\frac{c^{2}}{8 \pi h} I_{1} \int_{0}^{\infty} d v^{\prime} 1 \frac{1}{v^{2} v^{\prime}} a_{s}\left(v^{\prime}+v\right)-\frac{1}{v^{\prime 3}} \sigma_{s}\left(\left(v+v^{\prime}\right)\right] I_{0}\left(v^{\prime}\right), \text { (2) }
\end{aligned}
$$

where we have defined

$$
\sigma_{5 n}\left(u+h^{\prime}\right)=2 \pi !_{-!}^{1} d \mu_{0} \sigma_{5}\left(v+\nu^{\prime}, b_{0}\right) P_{n}\left(u_{0}\right)
$$

with $\mu_{0}=\Omega \cdot \Omega^{\prime}$. Here, $P_{n}\left(H_{0}\right)$ is the Legendre polynonial of $n^{\text {th }}$ decere. Also, the total scattering cross section is found fror:

$$
\sigma_{s}(v)=\sum_{0}^{\infty} d v^{\prime} \sigma_{s}\left(v \rightarrow v^{\prime}\right)
$$

Equations ( 80 ) and ( 81 ), together with appropriate initial and boundary conditions, form a closed set. Common boundary conditions used are known as the Marshak and Mark boundary conditions. The reader is referred to Ref. (9) for more details on these boundar: conditions.

Diffusion theory is helpful in that it represents the angular dependence of the transport equacion quite well. However, in problems where the radiation field is highly anisotropic, higher order terms in the expansion of the specific intensity can no longer be neglected. 


\subsubsection{Transport Approximations}

A more useful method for representation of the angular depenaence of the transport equation is the $P_{\Lambda}$ method. The greatest drawback of the Eddington diffusion technique is its limited arcuracy. In the $P_{N}$, or spherical harmonic method, any order of accuracy can theoretically be obtained. The $N$ in the $P_{R}$ method stands for the order of the approximation. As $N$ approaches infinity, the $P_{S}$ method gives the exact result. In practice for many years in neucron cransport problems, it was found that relatively low order approximations yield quite satisfactory results for many problems. (42)

A derivation of the $P_{N}$ equations sor radiation transport which included the induced scatering terms was not found in the literature. A detailed derivation follows.

We consider the complete derivation for slab geometry. The necessary adjustments are made for spherical geometry. Azimuthal symmetry is assumed. Hence, for slab and spherical geometry

$$
\begin{aligned}
& I(\underline{r}, v, \underline{\Omega}, t) \rightarrow I(r, v, H, t) \text { spherical } \\
& I(\underline{r}, v, \underline{\Omega}, t) \rightarrow I(z, v, \nu, t) \text { slab. }
\end{aligned}
$$

The intensity is expanded in an infinite series using orthogonal polynomials as a basis. Legendre polynomials are used. This expansion can be written as 


$$
I(z, v, \mu, t)=\sum_{n=0}^{\infty} I_{n}(z, v, t) P_{n}(\mu) \frac{(2 n+1)}{4 \pi}
$$

The physical significance of several of the expansion coefficients, $I_{n}(z, \nu, t)$, can be seen by using Eqs. (66)-(68). The radiative energy density per unit frequency is

$$
E(z, v, t)=\frac{2 \pi}{c} \int_{-1}^{1} d \mu \sum_{n=0}^{\infty} I_{n}(z, v, \tau) P_{n}(u) \frac{(2 n+1)}{4 \pi} .
$$

Using the orthogonality condition(27)

$$
\begin{aligned}
\int_{-1}^{1} d \mu P_{n}(\mu) P_{m}(\mu) & =0 \quad m \neq n \\
& =\frac{2}{2 n+1} \quad n=m
\end{aligned}
$$

and the fact that $P_{0}(\mu)=1, P_{1}(\mu)=\mu$, etc., we have

$$
\begin{aligned}
E(z, v, t) & =\frac{1}{c} \sum_{n=0}^{\infty} I_{n}(z, v, t) \frac{2 n+1}{2} \delta_{n 0} \frac{2}{2 n+1} \\
& =\frac{1}{c} I_{0}(z, v, t) .
\end{aligned}
$$

For the r-component of the flux per unit frequency we have

$$
\begin{aligned}
F(r, \nu, t) & =2 \pi \int_{-1}^{l} d \mu \sum_{n=0}^{\infty} I_{n}(r, \nu, t) P_{n}(\mu) \mu \frac{2 n+1}{4 \pi} \\
& =\sum_{n=0} I_{n}(r, \nu, t) \delta_{n 1}
\end{aligned}
$$




$$
=I_{1}(r, \nu, t) \text {. }
$$

The scattering kernel, $\sigma_{s}\left(\nu^{\prime}+\nu, \underline{\Omega}^{\prime} \cdot \underline{\Omega}\right)$, is also expanded in an Infinite series using Legengre polynomials as a basis, i.e.,

$$
\sigma_{s}\left(v^{\prime}+v, \underline{\Omega^{\prime}} \cdot \underline{\Omega}\right)=\sum_{n=0}^{\infty} \frac{2 n+1}{4 \pi} \sigma_{s n}\left(v^{\prime}+v\right) P_{n}\left(\underline{\Omega}^{\prime} \cdot \underline{\Omega}\right)
$$

The factor $P_{n}\left(\underline{\Omega}{ }^{\prime}+\Omega\right)$ can be rewritten using the addition formula for Legendre polynomials, namely, (27)

$$
\left.P_{n^{\prime}} \underline{\Omega}^{\prime} \cdot \underline{\Omega}\right)=P_{n}(\mu) P_{n}\left(\mu^{\prime}\right)+2 \sum_{m=1}^{\infty} \frac{(n-m) !}{(n+m) !} P_{n}^{m}(\mu) P_{n}^{m}\left(\mu^{\prime}\right) \cos \left[m\left(\phi-\phi^{\prime}\right)\right]
$$

where $\mathrm{P}_{\mathrm{n}}^{\mathrm{m}}(\mu)$ are the associated Legendre polynomials. It is important to note that

$$
\int_{0}^{2 \pi} d \phi^{\prime} \cos \left[m\left(\varphi-\phi^{\prime}\right)\right]=0 \quad m=1,2, \ldots
$$

The general equation of radiative transfer is

$$
\begin{aligned}
& \frac{1}{c} \frac{\partial I}{\partial t}+\underline{\Omega} \cdot \nabla I=\sigma_{a}^{\prime}(\nu)[B(\nu)-I(\nu, \underline{\Omega})] \\
& \left.+\int_{0}^{\infty} d v^{\prime} \int_{4 \pi} d \Omega \Omega^{\prime} \frac{v}{v^{\prime}} \sigma_{s}\left(v^{\prime}+v, \underline{\Omega^{\prime}} \cdot \underline{\Omega}\right) I\left(v^{\prime}, \Omega^{\prime}\right) 11+\frac{c^{2}}{2 h v^{3}} I(\nu, \underline{\Omega})\right] \\
& -\int_{0}^{\infty} d v^{\prime} \int_{4 \pi} d \Omega^{\prime} \sigma_{s}\left(v+v^{\prime}, \underline{\Omega} \cdot \underline{\Omega}^{\prime}\right) I(v, \underline{\Omega})\left[1+\frac{c^{2}}{2 h v^{\prime 3}} I\left(v^{\prime}, \underline{\Omega^{\prime}}\right)\right] \text {. }
\end{aligned}
$$


For spherical symmetry, the quantity $\Omega \cdot \nabla I$ must be evaluated.

$$
\underline{\Omega} \cdot \nabla I=\frac{\partial I}{\partial s}=\frac{\partial I}{\partial r} \frac{d r}{d s}+\frac{\partial I}{\partial \mu} \frac{d \mu}{d s}
$$

We have $\cos \theta=\frac{d r}{d s}=\mu$ and $\sin \theta=-r \frac{d \theta}{d s}$. (see Figure 1) Also,

$$
\begin{aligned}
\frac{d \mu}{d s} & =-\sin \theta \frac{d \theta}{d s}=-\sin \theta \frac{-\sin \theta}{r} \\
& =\frac{\sin ^{2} \theta}{r}=\frac{1-\mu^{2}}{r} .
\end{aligned}
$$

Hence,

$$
\frac{\partial I}{\partial s}=\mu \frac{\partial I}{\partial r}+\frac{\left(1-\mu^{2}\right)}{r} \frac{\partial I}{\partial \mu}
$$

For slab geometry, this quantity is much easier to evaluate.

$$
\frac{\partial I}{\partial s}=\frac{\partial I}{\partial z} \frac{d z}{d s}+\frac{\partial I}{\partial \mu} \frac{d \mu}{d s}
$$

where $\frac{d z}{d s}=\cos \theta=\mu$ and $\frac{d \mu}{d s}=0$. Hence, we have for slab geometry, (see Figure 2)

$$
\underline{\Omega} \cdot \nabla I=\mu \frac{\partial I}{\partial z} .
$$




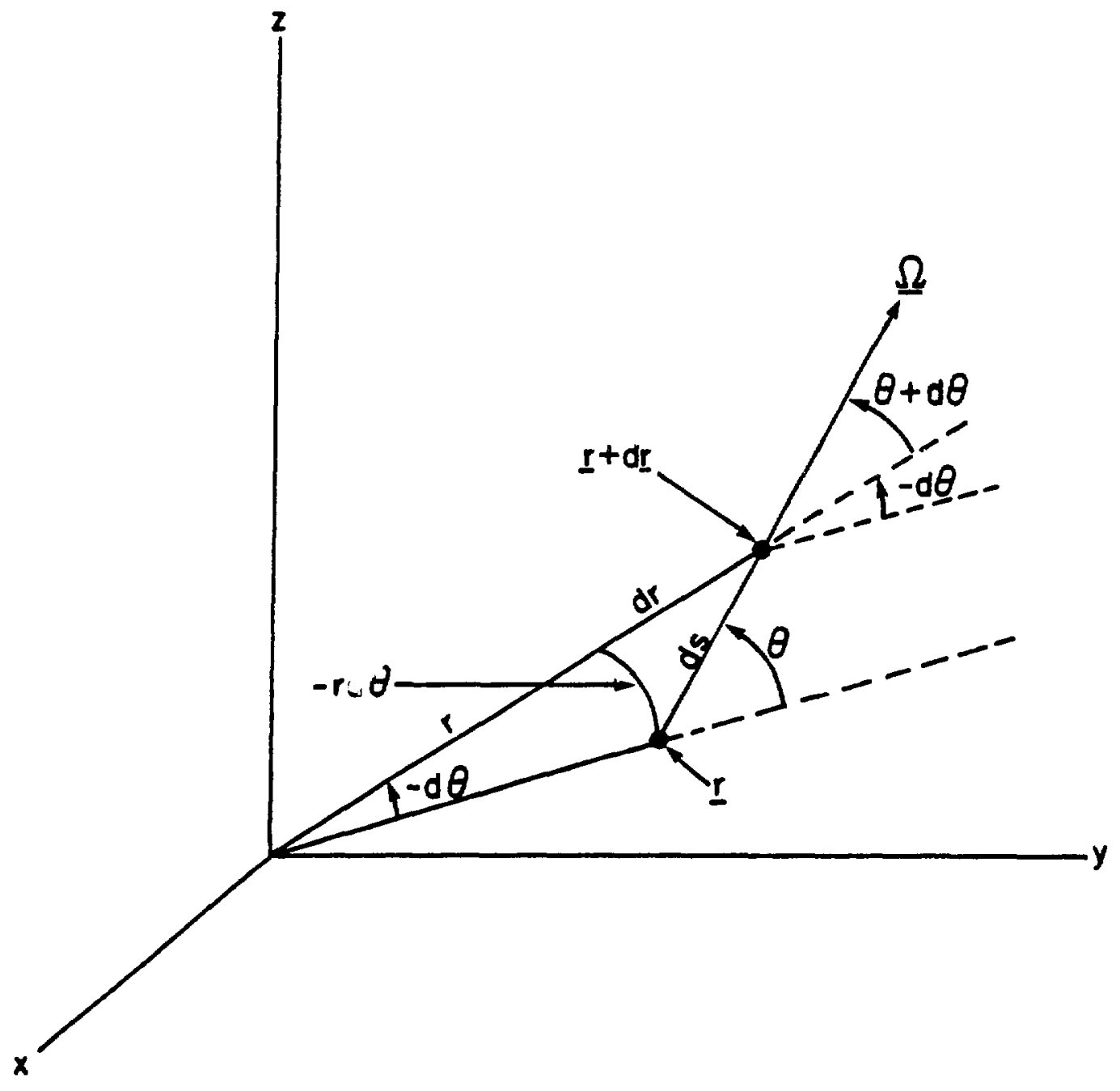

Figure 1 .

Spherical geometry. 


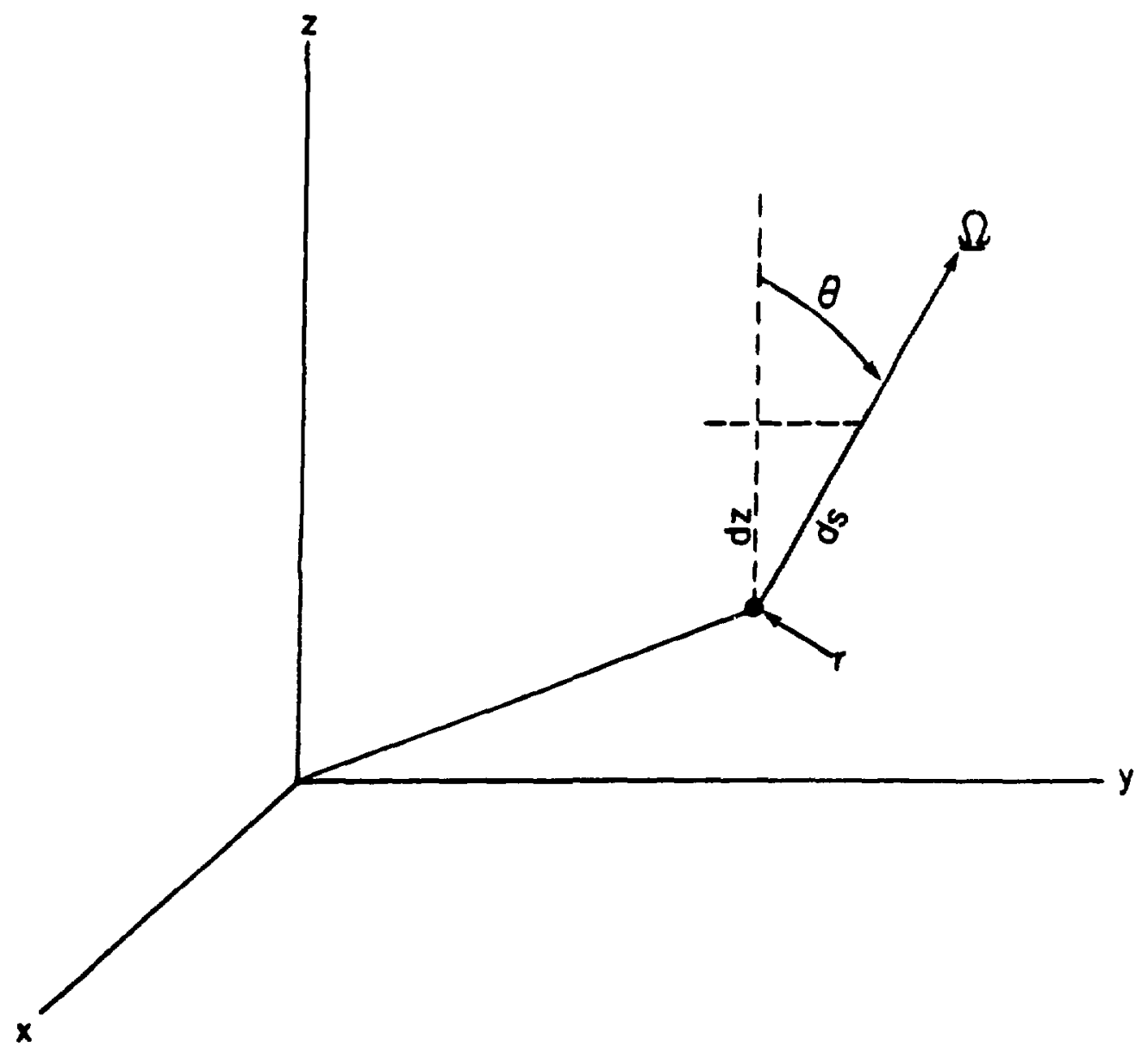

Figure 2.

Slab geometry. 
For simplicity, let $I(z, \nu, \mu, t)=I(\nu, \mu)$. Note that $I$ is independent of $\phi$ due to azimuthal symmetry.

Equation (92) for slab geometry can be written as

$$
\begin{aligned}
& \frac{1}{c} \frac{\partial I}{\partial t}(\nu, \mu)+\mu \frac{\partial I}{\partial z}(\nu, \mu)=\sigma_{a}^{\prime}(\nu)[B(\nu)-I(\nu, \mu)] \\
& +\int_{0}^{\infty} d v^{\prime} \frac{\nu}{v^{\prime}} \int_{4 \pi} \mathrm{d} \Omega^{\prime} \sigma_{s}\left(v^{\prime}+v, \underline{\Omega}^{\prime} \cdot \Omega\right) I\left(v^{\prime}, \mu^{\prime}\right) \\
& +\frac{c^{2}}{2 h} \int_{0}^{\infty} d v^{\prime} \frac{1}{v^{\prime} v^{2}}\left\{\pi d \Omega^{\prime} \sigma_{s}\left(\nu^{\prime}+\nu, \Omega^{\prime} \cdot \Omega\right) I\left(\nu^{\prime}, \mu^{\prime}\right) I(\nu, \mu)\right. \\
& -\int_{0}^{\infty} d v^{\prime} \int_{4 \pi} d \Omega^{\prime} \sigma_{s}\left(v \rightarrow v^{\prime}, \Omega \cdot \Omega^{\prime}\right) I(v, \mu) \\
& -\frac{c^{2}}{2 h} \int_{0}^{\infty} d v^{\prime} \frac{1}{v^{\prime} 3} \int_{4 \pi} d \Omega^{\prime} \sigma_{s}\left(\nu+\nu^{\prime}, \underline{\Omega} \cdot \Omega^{\prime}\right) I(\nu, \mu) I\left(\nu^{\prime}, \mu^{\prime}\right) \text {. }
\end{aligned}
$$

Consider the second term on the right hand side of Eq. (98).

$$
\begin{aligned}
& \int_{0}^{\infty} d \nu^{\prime} \frac{\nu}{\nu^{\prime}} \int_{-1}^{1} d \mu^{\prime} I\left(\nu^{\prime}, \mu^{\prime}\right) \sum_{n=0}^{\infty} \frac{(2 n+1)}{2} \sigma_{\mathrm{sn}}\left(\nu^{\prime}+\nu\right) P_{n}(\mu) P_{n}\left(\mu^{\prime}\right) \\
& =\sum_{n=0}^{\infty} \frac{2 n+1}{2} P_{n}(\mu) \int_{0}^{\infty} d \nu^{\prime} \frac{\nu}{\nu^{\prime}} \sigma_{s n}\left(\nu^{\prime} \rightarrow \nu\right) \int_{-1}^{1} d \mu^{\prime} P_{n}\left(\mu^{\prime}\right) \\
& \times \sum_{m=0}^{\infty} I_{m}\left(\nu^{\prime}\right) \frac{2 m+1}{4 \pi} P_{m}\left(\mu^{\prime}\right) \\
& =\sum_{n=0}^{\infty} \frac{2 n+1}{8 \pi} P_{n}(\mu) \int_{0}^{\infty} \mathrm{d} v^{\prime} \frac{v}{v^{\prime}} \sigma_{s n}\left(v^{\prime}+v\right) \sum_{t=0}^{\infty}(2 m+1) \\
& \times\}_{-1}^{1} d \mu^{\prime} P_{n}\left(\mu^{\prime}\right) P_{m}\left(\mu^{\prime}\right) I_{m}\left(\nu^{\prime}\right) \\
& =\sum_{n=0}^{\infty} \frac{2 n+1}{4 \pi} P_{n}(\mu) \int_{0}^{\infty} d v^{\prime} \frac{v}{\nu^{\prime}} \sigma_{s n}\left(\nu^{\prime}+v\right) I_{n}\left(\nu^{\prime}\right) \text {. }
\end{aligned}
$$

46 
Now consider the third term on the right hand side.

$$
\begin{gathered}
\frac{c^{2}}{2 h} I(\nu, \mu) \int_{0}^{\infty} d \nu^{\prime} \frac{1}{v^{\prime} v^{2}} \int_{4 \pi} d \Omega^{\prime} \sigma_{s}\left(\nu^{\prime}+\nu, \Omega \cdot \Omega^{\prime}\right) I\left(\nu^{\prime}, \mu^{\prime}\right) \\
=\frac{c^{2}}{2 h} I(\nu, \mu) \sum_{n=0}^{\infty} \frac{2 n+1}{4 \pi} P_{n}(\mu) \int_{0}^{\infty} d v^{\prime} \frac{1}{v^{\prime} v^{2}} \sigma_{s n^{\prime}}\left(\nu^{\prime}+v\right) I_{n}\left(\nu^{\prime}\right) \\
=\frac{c^{2}}{32 \pi^{2} h} \sum_{m=0}^{\infty} \sum_{n=0}^{\infty}(2 m+1)(2 n+1) P_{m}(\mu) P_{n}(\mu) I_{m}(\nu) \\
\times \int_{0}^{\infty} d v^{\prime} \frac{1}{v^{\prime} v^{2}} \sigma_{s n}\left(v^{\prime}+v\right) I_{n}\left(\nu^{\prime}\right) .
\end{gathered}
$$

The fourth term on the right hand side is

$$
\begin{gathered}
\int_{0}^{\infty} \mathrm{d} v^{\prime} \int_{4 \pi} \mathrm{d} \Omega^{\prime} \sigma_{\mathrm{s}}\left(\nu \rightarrow v^{\prime}, \Omega \cdot \Omega^{\prime}\right) I(\nu, \mu) \\
=I(\nu, \mu) \int_{0}^{\infty} \mathrm{d} v^{\prime} \int_{-1}^{1} \mathrm{~d} \mu^{\prime} \sum_{\mathrm{n}=0}^{\infty} \frac{2 \mathrm{n}+1}{2} \sigma_{\mathrm{sn}}\left(\nu+\nu^{\prime}\right) \mathrm{P}_{\mathrm{n}}(\mu) \mathrm{P}_{\mathrm{n}}\left(\mu^{\prime}\right) \\
=\sum_{\mathrm{m}=0}^{\infty} \frac{1}{8 \pi}(2 \mathrm{~m}+1) \sum_{\mathrm{n}=0}^{\infty}(2 \mathrm{n}+1) \mathrm{P}_{\mathrm{m}}(\mu) \mathrm{P}_{\mathrm{n}}(\mu) \mathrm{I}_{\mathrm{m}}(\nu) \\
\times \int_{0}^{\infty} \mathrm{d} v^{\prime} \sigma_{\mathrm{sn}}\left(\nu \rightarrow v^{\prime}\right) \int_{-1}^{1} \mathrm{~d} \mu^{\prime} \mathrm{P}_{\mathrm{n}}\left(\mu^{\prime}\right) .
\end{gathered}
$$

Finally, the last term can be written immediately with a previous result as

$$
\frac{c^{2}}{32 \pi^{2} h} \sum_{m=0}^{\infty} \sum_{n=0}^{\infty}(2 m+1)(2 n+1) P_{m}(\mu) P_{n}(\mu) I_{m}(\nu)
$$




$$
x \int_{0}^{\infty} d v^{\prime} \frac{1}{v^{\prime 3}} \sigma_{s n}\left(v \rightarrow v^{\prime}\right) I_{n}\left(v^{\prime}\right)
$$

Hence, Eq. (98) becomes

$$
\begin{aligned}
& \frac{1}{c} \frac{\partial I}{\partial t}(\nu, \mu)+\mu \frac{\partial I}{\partial z}(\nu, \mu)=\sigma_{a}^{\prime}(\nu)[B(\nu)-I(\nu, \mu)] \\
& +\sum_{n=0}^{\infty} \frac{2 n+1}{4 \pi} P_{n}(u) \int_{0}^{\infty} d \nu^{\prime} \frac{\nu}{\nu^{\prime}} \sigma_{s n}\left(\nu^{\prime}+\nu\right) I_{n}\left(\nu^{\prime}\right) \\
& +\frac{c^{2}}{32 \pi^{2} h} \sum_{m=0}^{\infty} \sum_{n=0}^{\infty}(2 m+1)(2 n+1) P_{m}(\mu) P_{n}(\mu) I_{m}(v) \\
& x \int_{0}^{\infty} d v^{\prime} \frac{1}{v^{\prime} v^{2}} \sigma_{\operatorname{sn}}\left(v^{\prime}+v\right) I_{n}\left(v^{\prime}\right) \\
& -\sum_{m=0}^{\infty} \frac{1}{8 \pi}(2 m+1) \sum_{n=0}^{\infty}(2 n+1) P_{m}(\mu) P_{n}(\mu) I_{m}(\nu) \\
& x \int_{0}^{\infty} d v^{\prime} \sigma_{s n}\left(v+v^{\prime}\right) \int_{-1}^{1} d \mu^{\prime} P_{n}\left(\mu^{\prime}\right) \\
& -\frac{c^{2}}{32 \pi^{2} h} \sum_{m=0}^{\infty} \sum_{n=0}^{\infty}(2 m+1)(2 n+1) P_{m}(\mu) P_{n}(\mu) I_{m}(\nu) \\
& \times \int_{0}^{\infty} d v^{\prime} \frac{1}{v^{\prime 3}} \sigma_{s n}\left(v \rightarrow v^{\prime}\right) I_{n}\left(v^{\prime}\right) \text {. }
\end{aligned}
$$

We now operate on Eq. (103) with $\int_{-1}^{1} d u$ to get 


$$
\begin{aligned}
& \sum_{n=0}^{\infty} \frac{2 \pi+1}{4 \pi} \int_{-1}^{1} d \mu P_{n}(\mu)\left[\frac{1}{c} \frac{\partial I_{n}(\nu)}{\partial t}+\mu \frac{\partial I_{n}(\nu)}{\partial z}\right] \\
& =\sigma_{a}^{\prime}(\nu)\left[2 B(\nu)-\sum_{n=0}^{\infty} \frac{2 \pi+1}{4 \pi} I_{n}(\nu) \int_{-1}^{1} d \mu P_{n}(\mu)\right] \\
& +\sum_{n=0}^{\infty} \frac{2 \pi+1}{4 \pi} \int_{0}^{\infty} d v^{\prime} v_{v^{\prime}}^{\nu} \sigma_{s n}\left(v^{\prime}+v\right) I_{n}\left(v^{\prime}\right) \int_{-1}^{1} d \mu P_{n}(\mu) \\
& +\frac{c^{2}}{16 \pi^{2} h} \sum_{n=0}^{\infty}(2 n+1) I_{n}(v) \int_{0}^{\infty} d v^{\prime} 1 \frac{1}{v^{\prime} v^{2}} \sigma_{s n}\left(v^{\prime}+\nu\right)- \\
& \left.\frac{1}{v^{*}} \sigma_{s n}\left(\nu+v^{*}\right)\right] I_{n}\left(\nu^{\prime}\right) \\
& -\sum_{n=0}^{\infty} \frac{2 n+1}{4 \pi} I_{n}(\nu) \int_{-1}^{1} d \mu^{\prime} P_{n}\left(\mu^{\prime}\right) \int_{0}^{\infty} d v^{\prime} \sigma_{s n}\left(\nu \rightarrow v^{\circ}\right) .
\end{aligned}
$$

This becomes

$$
\begin{aligned}
& \sum_{n=0}^{\infty}(2 n+1) \int_{-1}^{1} d \mu P_{n}(\nu)\left[\int_{c}^{1} \frac{\partial I_{n}(\nu)}{\partial t}+\mu \frac{\partial I_{n}(\nu)}{\partial z}\right] \\
& =\sigma_{a}(\nu)\left[8 \pi B(\nu)-\sum_{n=0}^{\infty}(2 n+1) I_{n}(\nu) \int_{-1}^{1} d \mu P_{n}(\mu)\right] \\
& +\sum_{n=0}^{\infty}(2 n+1)\left[\int _ { - 1 } ^ { 1 } d \mu P _ { n } ( \mu ) \left(\int_{0}^{\infty} d \nu^{\prime} \frac{\nu}{\nu^{\prime}} \sigma_{s n}\left(\nu^{\prime} \rightarrow \nu\right) I_{n}\left(\nu^{\prime}\right)\right.\right. \\
& -I_{n}(\nu) \sigma_{s}(\nu) \\
& +\frac{c^{2}}{4 \pi h} I_{n}(\nu) \int_{0}^{\infty} d \nu^{\prime}\left[\frac{1}{\nu^{\prime} v^{2}} \sigma_{s n}\left(\nu^{\prime}+\nu\right)\right. \\
& \left.\left.-\frac{1}{v^{\prime} 3} \sigma_{\operatorname{sn}}\left(v+v^{\prime}\right)\right] I_{n}\left(v^{\prime}\right)\right] \text {. }
\end{aligned}
$$

49 


$$
\left.\left.-\frac{1}{v^{\prime 3}} \sigma_{\operatorname{sn}}\left(v+v^{\prime}\right)\right] I_{n}\left(v^{\prime}\right)\right]
$$

This gives the first equation of the $P_{N}$ equations for slab geometry, namely,

$$
\begin{gathered}
\left.\frac{1}{c}{\frac{\partial I_{0}}{\partial t}}^{(v)}+{\frac{\partial I_{1}}{\partial z}}^{(v)}=\sigma_{a}^{\prime}(v) \mid 4 \pi B(v)-I_{0}(v)\right] \\
-I_{0}(v) \sigma_{s}(v)+\int_{0}^{\infty} d v^{\prime} \frac{v}{v^{\prime}} \sigma_{s 0}\left(v^{\prime}+v\right) I_{0}\left(v^{\prime}\right) \\
+\frac{c^{2}}{8 \pi h} \sum_{n=0}^{\infty}(2 n+1) I_{n}(v) \int_{0}^{\infty} d v^{\prime}\left[\frac{1}{v^{\prime} v^{2}} \sigma_{s n}\left(v^{\prime}+v\right)\right. \\
\left.-\frac{1}{v^{\prime 3}} \sigma_{s n}\left(v \rightarrow v^{\prime}\right)\right] I_{n}\left(v^{\prime}\right) .
\end{gathered}
$$

In order to get the rest of the $\mathrm{P}_{\mathrm{N}}$ equations, we operate on Eq. (103) with the integral operator $\int_{-1}^{1} d \mu P_{j}(\mu)$. This yields the expression

$$
\begin{aligned}
& \frac{1}{2 \pi c} \frac{\partial I_{j}(\nu)}{\partial t}+\sum_{n=0}^{\infty} \frac{2 n+1}{4 \pi} \frac{\partial I_{n}(\nu)}{\partial z} \int_{-1}^{1} d \mu \mu P_{n}(\mu) P_{j}(\nu) \\
& =\sigma_{a}^{\prime}(v)\left[\frac{-1}{2 \pi} I_{j}(\nu)\right]+\frac{1}{2 \pi} \int_{0}^{\infty} d v^{\prime} \frac{v}{v^{\prime}} \sigma_{s j}\left(v^{\prime}+v\right) I_{j}\left(v^{\prime}\right) \\
& -\sum_{m=0}^{\infty} \frac{2 m+1}{4 \pi} \int_{-1}^{1} d \mu P_{m}(\mu) P_{j}(\mu) I_{m}(\nu) \sigma_{s}(v) \\
& +\frac{c^{2}}{32 \pi^{2} h} \sum_{m=0}^{\infty} \sum_{n=0}^{\infty} I_{m}(v)(2 m+1)(2 n+1) \int_{0}^{\infty} d v^{\prime} 1 \frac{1}{v^{\prime} v^{2}} \sigma_{s n}\left(v^{\prime}+v\right)
\end{aligned}
$$




$$
\left.-\frac{1}{\nu^{\prime 3}} \sigma_{s n}\left(\nu \rightarrow \nu^{\prime}\right)\right] I_{n}\left(\nu^{\prime}\right) \int_{-1}^{1} d \mu P_{n}(\mu) P_{m}(\mu) P_{j}(\nu)
$$

Equation (107) can be rewritten as

$$
\begin{aligned}
& \frac{1}{c} \frac{\partial I_{j}(\nu)}{\partial t}+\sum_{n=0}^{\infty} \frac{1}{2} \frac{\partial I_{n}(\nu)}{\partial z} \int_{-1}^{1} d \mu P_{j}(\mu)\left[(n+1) P_{n+1}(\mu)+n P_{n-1}(\mu)\right] \\
& =-\sigma_{a}^{\prime}(\nu) I_{j}(\nu)+\int_{0}^{\infty} d \nu^{\prime} \frac{\nu}{\nu^{\prime}} \sigma_{s j}\left(\nu^{\prime}+\nu\right) I_{j}\left(\nu^{\prime}\right)-I_{j}(\nu) \sigma_{s}(\nu) \\
& +\frac{c^{2}}{16 \pi h} \sum_{m=0}^{\infty} \sum_{n=0}^{\infty}(2 m+1)(2 n+1) I_{m}(\nu) \int_{0}^{\infty} d \nu^{\prime}\left[\frac{1}{\nu^{\prime} v^{2}} \sigma_{s n}\left(\nu^{\prime}+\nu\right)\right. \\
& \left.-\frac{1}{v^{\prime} 3} \sigma_{s n}\left(\nu \rightarrow \nu^{\prime}\right)\right] I_{n}\left(\nu^{\prime}\right) \int_{-1}^{1} d \mu P_{n}(\mu) P_{m}(\mu) P_{j}(\mu)
\end{aligned}
$$

for $j=1,2, \ldots$

This gives the set of equations which, in conjunction with Eq. (106), complete the $P_{N}$ equations.

$$
\begin{aligned}
& \frac{2 j+1}{c}{\frac{\partial I_{j}}{\partial t}}^{(\nu)}+j{\frac{\partial I_{j-1}}{\partial z}(\nu)}^{(j+1)} \frac{\partial I_{j+1}}{\partial z}(\nu) \\
& +I_{j}(\nu) \sigma_{s}(\nu)(2 j+1)+(2 j+1) \sigma_{a}^{\prime}(\nu) I_{j}(\nu)= \\
& (2 j+1) \int_{0}^{\infty} d v^{\prime} \frac{\nu}{v^{\prime}} \sigma_{6 j}\left(v^{\prime}+v\right) I_{j}\left(v^{\prime}\right)+\frac{2 j+1}{16 \pi h} c^{2} \sum_{m=0}^{\infty} \sum_{n=0}^{\infty}(2 m+1)(2 n+1) \\
& \times I_{m}(v) \int_{0}^{\infty} d v^{\prime}\left[\frac{1}{v^{\prime} v^{2}} \sigma_{s n}\left(v^{\prime}+v\right)-\frac{1}{v^{\prime 3}} \sigma_{s n}\left(v+v^{\prime}\right)\right] I_{n}\left(v^{\prime}\right) \\
& \times \int_{-1}^{1} d \mu P_{n}(\mu) P_{m}(\mu) P_{j}(\mu)
\end{aligned}
$$


where $j=1,2,3 \ldots$

Equations (109) and (106) are the $P_{N}$ equations for slab geometry. Since only the partial derivative terms are different for the problem of spherical symetry, we can imnediately write, usiag previous results,

$$
\begin{aligned}
& \frac{2}{c} \frac{\partial I_{0}(v)}{\partial t}+2 \frac{\partial I_{1}(v)}{\partial r}+\sum_{n=0}^{\infty} \frac{2 n+1}{r} I_{n}(v) \sum_{-1}^{1} d u\left(1-\nu^{2}\right) \frac{d D_{n}(u)}{d t} \\
& =\sigma_{a}^{\prime}(v)\left[8 \pi B(v)-2 I_{0}(v)\right]-2 I_{0}(v) \sigma_{s}(v) \\
& +2 \int_{0}^{\infty} d v^{\prime} \frac{v}{v^{\prime}} \sigma_{s v^{\prime}}\left(v^{\prime}+v\right) I_{0}\left(v^{\prime}\right)+\frac{c^{2}}{4 \pi h} \sum_{n=0}^{\infty}(2 n+1) I_{r}(v) \\
& \quad \int_{0}^{\infty} d v^{\prime}\left[\frac{1}{v^{\prime} v^{2}} \sigma_{s n^{\prime}}\left(v^{\prime} \rightarrow v^{\prime}\right)-\frac{1}{v^{\prime 3}} \sigma_{s n}\left(v \rightarrow v^{\prime}\right)\right] I_{n}\left(v^{\prime}\right) .
\end{aligned}
$$

We now rake use of the identity (27)

$$
\left(\mu^{2}-1\right){\frac{d P_{n}}{d \mu}}^{(\mu)}=\frac{n(n+1)}{2 n+1}\left[P_{n+1}(\mu)-P_{n-1}(\mu)\right],
$$

and orthogonality to get

$$
\begin{aligned}
& \frac{1}{c}{\frac{\partial I_{0}}{\partial t}(v)}+{\frac{\partial I_{1}}{\partial r}(v)}^{2}+\frac{2}{r} I_{1}(v)=\sigma_{a}^{\prime}(v)\left[4 \pi B(v)-I_{0}(v)\right] \\
& -I_{0}(v) \sigma_{s}(v)+\int_{0}^{\infty} d v^{\prime} \frac{v}{v^{\prime}} \sigma_{s}\left(v^{\prime} \rightarrow v\right) I_{0}\left(v^{\prime}\right)
\end{aligned}
$$




$$
\begin{aligned}
+\frac{c^{2}}{8 \pi h} \sum_{n=0}^{\infty}(2 n+1) & I_{n}(v) \sum_{0}^{\infty} d v^{\prime} 1 \frac{1}{v^{\prime} v^{2}} \sigma_{s n}\left(v^{\prime}+v\right) \\
& \left.-\frac{1}{v^{\prime} 3} \sigma_{s n}\left(u \rightarrow v^{\prime}\right)\right] I_{n}\left(v^{\prime}\right)
\end{aligned}
$$

This is the first equation in the series for spherical symetry. Likewise, using previous results, we can immediately write the rest of the $P_{N}$ equations.

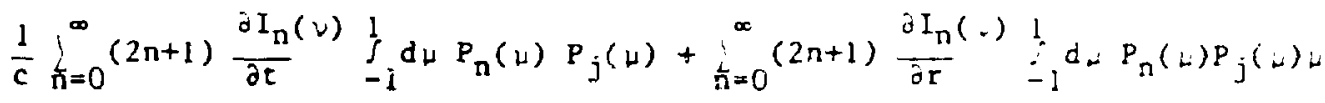

$$
\begin{aligned}
& +\sum_{n=0}^{\infty} \frac{I_{n}(\nu)}{r} n(n+1) \int_{-1}^{1} d u\left[P_{n-1}(u)-P_{n+j}(\nu)\right] P_{j}(\nu) \\
& +2 I_{j}(v) \sigma_{a}^{\prime}(v)=2 \int_{0}^{\infty} \frac{v}{v^{\prime}} \sigma_{s j}\left(v^{\prime}+v\right) I_{j}\left(v^{\prime}\right) \\
& -\sum_{m=0}^{\infty}(2 m+1) \int_{-1}^{1} d u P_{m}(u) P_{j}(u) I_{m}(v) \sigma_{s}(\nu) \\
& +\frac{c^{2}}{8 \pi h} \sum_{m=0}^{\infty} \sum_{n=0}^{\infty} I_{m}(\nu)(2 m+1)(2 n+1) \\
& x \int_{0}^{\infty} d v^{\prime}\left[\frac{1}{v^{\prime} v^{2}} \sigma_{s n}\left(v^{\prime}+v\right)-\frac{1}{v^{\prime} 3} \sigma_{s n}\left(v+v^{\prime}\right)\right] \\
& i_{n}\left(\nu^{\prime}\right) \int_{-1}^{1} d \nu P_{n}(\mu) P_{m}(\mu) P_{j}(\mu) \text {. }
\end{aligned}
$$

Again, making use of orthogonality and the identity (27)

$$
\mu P_{n}(\mu)=\frac{1}{2 n+1}\left[n P_{n-1}(\mu)+(n+1) P_{n+1}(\mu)\right]
$$


we get

$$
\begin{aligned}
& \frac{2 j+1}{c} \frac{\partial I j}{\partial t}(v)^{\partial t} \frac{\partial l_{j-l}(v)}{\partial r}+(j+1) \frac{\partial I j+l}{\partial r}(v) \\
& +(j+1)(j+2) \frac{I_{j+1}(v)}{r}-j(j-1) \frac{I_{j-1}(v)}{r}+(2 j+1) o_{a}^{\prime}(v) I_{j}(v)= \\
& (2 j+1) \int_{0}^{\infty} d v^{\prime} \frac{v}{v^{\prime}} \sigma_{s j}\left(v^{\prime}+v\right) I_{j}\left(v^{\prime}\right)-\sigma_{s}(v) I_{j}(v) \\
& +\frac{c^{2}}{16 \pi h}(2 j+1) \sum_{n=0}^{\infty} \sum_{m=0}^{\infty} I_{m}(v)(2 m+1)(2 n+1) \sum_{0}^{\infty} d v^{\prime} l \frac{1}{v^{\prime} v^{2}} \sigma_{s n}\left(v^{\prime} \rightarrow v\right) \\
& \left.-\frac{1}{v^{\prime} 3} \sigma_{s n}\left(v \rightarrow \nu^{\prime}\right)\right] I_{n}\left(\nu^{\prime}\right) \int_{-1}^{1} d u P_{n}(\mu) P_{m}(u) P_{j}(u)
\end{aligned}
$$

for $j=1,2, \ldots$ Equations $(115)$ and $(112)$ are the $P_{N}$ equations for problems with spherical symetry.

If the full set of equations were solved, the solution of the transport equation would be exact and contain all directional information. This is an impossible task. In order to get a solution, the $P_{N}$ equations are truncated after $N+1$ equations. For example, In spherical geometry, a $P_{3}$ problem would consist of Eq. (111) and Eq. (115) for $\mathrm{f}=1, \mathrm{j}=2$, and $\mathrm{j}=3$. A problem arises in the truncation of the $P_{N}$ equations. In a $P_{N}$ approximation, we are left with $N+1$ equations in $N+2$ dependent varlables, $I_{0}, I_{1}, \ldots, I_{N}, I_{N+1}$. The easiest way to close this set of equations is by setting 


$$
I_{N+1}(v)=0
$$

The boundary and Initial conditions for the $P_{N}$ equations can be found from Eqs. (38) and (39). Let these conditions be $I\left(r_{s}, v, \mu, t\right)$ and $I(r, v, \mu, 0)$ respectively.

Since

$$
I_{n}(r, v, t)=\int_{4 \pi} d \Omega I(r, v, \mu, t)
$$

the boundary and initial conditions for the expansion coefficients, $I_{n}$, can be found from

$$
I_{n}\left(r_{s}, v, t\right)=\int_{4 \pi} d \Omega I\left(r_{s}, v, \mu, t\right)
$$

and

$$
I_{n}(r, \nu, 0)=\int_{4 \pi} d \Omega I(r, \nu, \mu, 0)
$$

The $P_{N}$ method, alihough simplifying the transport equation substantially, still leaves a complex set of integro-differential equations to solve. Another representation of the frequency integrals is desirable. One method of accomplishing this is known as the multigroup method. A variation of this technique is used for the solutions obtalned in this thesis. The technique used in this thesis is discussed in Chapters Three and Four. 
Since the multigroup equations for the full transport equation can be found in the literature, (9) the multigroup formulation of the $P_{N}$ equations for spherical geometry will only be outlined here.

In the multigroup method, the frequency spectrum is divided into G frequency groups. This is 11 lustrated below.

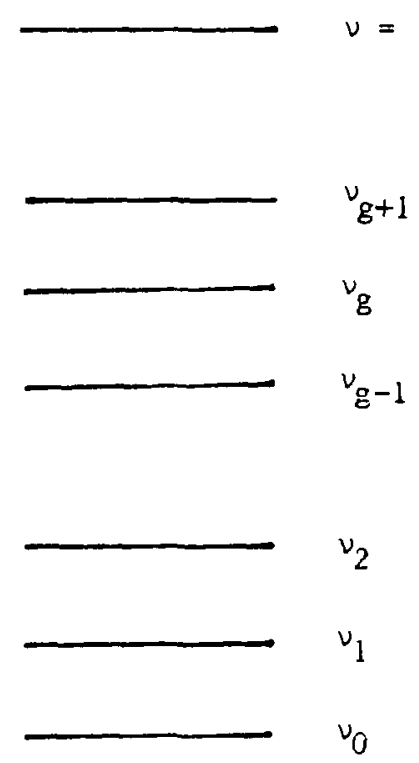

Each quantity is defined as a weighted average over each frequency group. Let

$$
\begin{aligned}
& I g=\int_{\nu_{g}-1}^{\nu} d v^{\prime} I_{f}\left(\nu^{\prime}\right), \\
& I_{a g}=\int_{\nu_{g-1}}^{\nu_{g}} d v^{\prime} \sigma_{a}^{\prime}\left(v^{\prime}\right) B\left(v^{\prime}\right),
\end{aligned}
$$




$$
\begin{aligned}
& \sigma_{a j g}^{\prime}=\frac{1}{I g} \int_{v_{g}-1}^{v_{g} d v^{\prime}} \sigma_{a}^{\prime}\left(v^{\prime}\right) I_{j}\left(v^{\prime}\right), \\
& \sigma_{s j g}=\frac{1}{I g} \int_{\nu_{g-1}}^{\nu_{g}} d v^{\prime} \sigma_{s}\left(v^{\prime}\right) I_{j}\left(v^{\prime}\right), \\
& \sigma_{s j}\left(g \rightarrow g^{\prime}\right)=\frac{1}{I g I g_{j}^{\prime}} \int_{v_{g-1}}^{v_{j}} d v I_{j}(v) \\
& \times \int_{v_{g}^{\prime}-1}^{\nu_{g}} d v^{\prime} \frac{v}{v^{\prime}} \sigma_{s j}\left(v^{\prime} \rightarrow \nu\right) I_{j}\left(v^{\prime}\right), \\
& g(n, \pi, j)=\int_{-1}^{1} d \mu P_{n}(\mu) P_{m}(\mu) P_{j}(\mu), \\
& A_{s n m}\left(g^{\prime}+g\right)=\frac{1}{I g I_{m}^{\prime}} \int_{n_{g-1}}^{\nu} d v I_{m}(v) \\
& \times \sum_{v_{g^{\prime}-1}}^{\nu_{g^{\prime}}} d v^{\prime} \frac{1}{v^{\prime} v^{2}} \sigma_{\mathrm{sn}}\left(v^{\prime}+v\right) I_{n}\left(v^{\prime}\right) \text {, }
\end{aligned}
$$

and

$$
\begin{aligned}
B_{s n m}\left(g+g^{\prime}\right)= & \frac{1}{I_{m}^{g} I_{n}^{\prime}} \int_{v_{g-1}}^{\nu} d v I_{m}(v) \\
& \times \int_{v_{g^{\prime}-1} v_{g^{\prime}} d v^{\prime}}^{\nu^{\prime}} \frac{1}{v^{\prime 3}} \sigma_{s n}\left(v+v^{\prime}\right) I_{n}\left(v^{\prime}\right)
\end{aligned}
$$


Utilizing these defintions, the frequency integrals can be approximated by sums over the $G$ frequency groups. Hence, Eqs. and (115) can be rewritten as

$$
\begin{aligned}
& \frac{1}{c} \frac{\partial I_{0}^{g}}{\partial t}+\frac{\partial I_{1}^{g}}{\partial r}+\frac{2}{r} I_{1}^{g}= \\
& 4 \pi \Gamma_{a g}-\sigma_{a 0 g}^{\prime} I_{0}^{g}-\sigma_{s 0 g} I_{0}^{g}+\sum_{g^{\prime}=1}^{G} \sigma_{s 0}\left(g^{\prime}+g\right) I_{0}^{g^{\prime}} \\
& +\frac{c^{2}}{8 \pi h} \sum_{n=0}^{\infty}(2 n+1) I_{n}^{8} \sum_{g^{\prime}=1}^{G}\left[A_{s n n}\left(g^{\prime}+g\right)-B_{s n n}\left(g^{+}+g^{\prime}\right)\right] I_{n}^{g^{\prime}}
\end{aligned}
$$

and

$$
\begin{aligned}
& \frac{2 j+1}{c} \frac{\partial I_{j}^{g}}{\partial t}+j \frac{\partial I_{j-1}^{g}}{\partial r}+(j+1) \frac{\partial I_{j+1}^{g}}{\partial r}+(j+1)(j+2) \frac{I_{j+1}^{g}}{r} \\
& -j(j-1) \frac{1}{r} I_{j-1}^{g}+(2 j+1) \sigma_{a j g}^{\prime} I_{j}^{g}+\sigma_{s j g} I_{j}^{g}= \\
& (2 j+1) \sum_{g^{\prime}=1}^{G} \sigma_{s j^{\prime}}\left(g^{\prime}+g\right) I_{j}^{g^{\prime}}+\frac{c^{2}}{I 6 \pi h}(2 j+1) \sum_{n=0}^{\infty} \sum_{m=0}^{\infty} g(n, m, j) \\
& \times(2 m+1)(2 n+1) I_{m}^{g} \sum_{g^{\prime}=1}^{G}\left[A_{s n m}\left(g^{\prime}+g\right)-B_{s n m}\left(g+g^{\prime}\right)\right] I_{n}^{g^{\prime}}
\end{aligned}
$$

for $j=1,2, \ldots$ and $g=1,2, \ldots, G$.

These are the $P_{N}$ multigroup equations for radiative transport with spherical symetry. Hore accuracy can be obtained by decreasing the frequency point spacing. In radiation transport problems, sharp spectral features are quite common. Hence, if a multigroup approach is used, a large number of frequency groups is a necessity. In the 
varlation of this method which is used in this thesis, between 135 and 200 groups were used.

If the induced scattering terms were absent, solutions of the $P_{n}$ multigroup equations could be accomplished by using finite difference techniques. (43) However, Induced scattering introduces terms which are quadratic in the expansion coefficients $I_{j}^{g}$. Simultaneous solution of the difference equations becomes extremely difficult. Hence, a different scheme is used to solve the $P_{X}$ equations. The method of characteristics, used in this thesis, is discussed in Chapter Three.

If the set of $P_{1}$ equations is closed by setting $P_{2}=0$, the $P_{1}$ equations are equivalant to the diffusion equations. We can write down the $P_{1}$ equations in slab geometry using Eqs. (106) and (109) for $j=1$. This gives the two equations

$$
\begin{aligned}
& \frac{1}{c}{\frac{\partial I_{0}}{\partial t}(v)}^{\frac{\partial I_{1}}{\partial z}}(v)=\sigma_{a}^{\prime}(v)\left[4 \pi B(v)-I_{0}(v)\right]-\sigma_{s}(v) I_{0}(v) \\
& +\int_{0}^{\infty} d v^{\prime} \frac{v}{v^{\prime}} \sigma_{s 0^{\prime}}\left(v^{\prime} \rightarrow v\right) I_{0}\left(v^{\prime}\right)+\frac{c^{2}}{8 \pi h} I_{0}(v) \int_{0}^{\infty} d v^{\prime}\left[\frac{1}{v^{2} v^{\prime}} \sigma_{s}\left(v^{\prime}+v\right)\right. \\
& \left.-\frac{1}{v^{\prime 3}} \sigma_{s 0}\left(v \rightarrow v^{\prime}\right)\right] I_{0}\left(v^{\prime}\right)+\frac{3 c^{2}}{8 \pi h} I_{1}(v) \int_{0}^{\infty} d v^{\prime}\left[\frac{1}{v^{2} v^{\prime}} \sigma_{s 1}\left(v^{\prime}+v\right)\right. \\
& \left.-\frac{1}{v^{\prime 3}} \sigma_{s 1}\left(v \rightarrow v^{\prime}\right)\right] I_{1}\left(v^{\prime}\right)
\end{aligned}
$$

and 


$$
\begin{aligned}
& \frac{1}{c} \frac{\partial I_{1}}{\partial t}+\frac{1}{3} \frac{\partial I_{0}(\nu)}{\partial z}+\left[\sigma_{a}^{\prime}+\sigma_{s}\right] I_{1}=\int_{0}^{\infty} d \nu^{\prime} \frac{\nu}{\nu^{\prime}} \sigma_{s l}\left(\nu^{\prime}+\nu\right) I_{1}\left(\nu^{\prime}\right) \\
& \left.+\frac{c^{2}}{8 \pi h} I_{0} \int_{0}^{\infty} d v^{\prime} 1 \frac{1}{v^{2} v^{\prime}} \sigma_{s 1}\left(v^{\prime}+v\right)-\frac{1}{v^{\prime 3}} \sigma_{s l}\left(v+v^{\prime}\right)\right] I_{1}\left(v^{\prime}\right) \\
& +\frac{c^{2}}{8 \pi h} I_{1} \int_{0}^{\infty} d v^{\prime}\left[\frac{1}{v^{2} v^{\prime}} \sigma_{s 0}\left(v^{\prime}+\nu\right)-\frac{1}{v^{\prime 3}} \sigma_{s 0}\left(\left(\nu \rightarrow v^{\prime}\right)\right] I_{0}\left(v^{\prime}\right)\right.
\end{aligned}
$$

Comparison of Eqs. (130) and (131) with Eqs. (80) and (81) demonstrates the equivalence of the $P_{1}$ approximation and the diffusion equations. The advantage is that the same techniques used to solve the $P_{1}$ equations can be applied to higher order $P_{: i}$ approximations with few modifications. This yields more accurate directional information. Also, the $P_{N}$ equations might be improved by truncating the series in a manner other than setting $I_{N+1}(v)=0$. This, in the $P_{1}$ example, would correspond to the variable Eddington diffusion approximation with induced scattering included.

This gives a brief list of some approaches used to address the radiation transport equation. This thesis utilizes the $P_{N}$ method as outlined in this chapter. 
3. METHOD OF CHARACTERISTICS IN RADIATION TKANSPORT

The nonlinearity in the $P_{N}$ equations limits the available techniques which can be used to solve them. One method which can be considered is the method of characteristics. This method is ideally suited for handing nonlinearities. A general discussion of tilis method can be found in Appendix I. In order to utilize the method of characteristics, the equations to be solved must be hyperbolic. The requirements for hyperbolicity can also be found in the Appendix I.

\subsection{Reduction to Canonical Form}

The starting point for solution of the radiation transport equation will be the $P_{N}$ equations for spherical symmetry derived in Chapter Two. In order to test the $P_{N}$ equations for hyperbolicity, we write them as

$$
\frac{2 j+1}{c} \frac{\partial I}{\partial t}(\nu)^{2}+j \frac{\partial I}{\partial r}(\nu)^{\partial r}+(j+1){\frac{\partial I_{j+1}}{\partial r}}^{(\nu)}+b_{j}(\underline{r}, \nu, \underline{I}, t)=0
$$

for $j=0,1,2, \ldots$ and

$$
\underline{I}=\left(I_{0}, I_{1}, I_{2}, \ldots\right) .
$$


Consider the $P_{1}$ equations written in vector form.

$$
\frac{\partial}{\partial \tau}\left(\begin{array}{l}
I_{0} \\
I_{1}
\end{array}\right)+\left(\begin{array}{cc}
0 & c \\
\frac{c}{3} & 0
\end{array}\right) \frac{\partial}{\partial r}\left(\begin{array}{l}
I_{0} \\
I_{1}
\end{array}\right)+\left(\begin{array}{l}
b_{0} \\
b_{1}
\end{array}\right)=0
$$

We let

$$
A=\left(\begin{array}{ll}
0 & c \\
c / 3 & 0
\end{array}\right)
$$

Also,

$$
\hat{I}=\left(\begin{array}{ll}
1 & 0 \\
0 & 1
\end{array}\right)
$$

is the identity matrix. Then, if we set

$$
\operatorname{det}|A-\lambda \hat{I}|=0 \text {, }
$$

and solve for the possible values of $\lambda$, we get

$$
\lambda^{2}-\frac{c^{2}}{3}=0
$$

or

$$
\lambda= \pm \frac{c}{\sqrt{3}}
$$


Hence, the $P_{1}$ equations are indeed hyperbolic.

Considering the $\mathrm{P}_{2}$ equations in vector form, we can write

$$
\frac{\partial}{\partial t}\left(\begin{array}{l}
I_{0} \\
I_{1} \\
I_{2}
\end{array}\right)+\left(\begin{array}{lcc}
0 & c & 0 \\
c / 3 & 0 & 2 c / 3 \\
0 & 2 c / 5 & 0
\end{array}\right) \frac{\partial}{\partial r}\left(\begin{array}{l}
I_{0} \\
I_{1} \\
I_{2}
\end{array}\right)+\left(\begin{array}{l}
b_{0} \\
b_{1} \\
D_{2}
\end{array}\right)=0
$$

With

$$
A=\left(\begin{array}{llc}
0 & 0 & 0 \\
c / 3 & 0 & 2 c / 3 \\
0 & 2 c / 5 & 0
\end{array}\right)
$$

we set

$$
\operatorname{det}|A-\lambda \hat{I}|=0
$$

and obtain the relation

$$
\lambda^{3}+\frac{9 c^{2}}{15} \lambda=0
$$

This yields the values

$$
\lambda=0, \pm c(3 / 5)^{1 / 2} \text {. }
$$

The zero value for $\lambda$ introduces a parabolic nature into the equations.

The $P_{3}$ equations in vector form are 


$$
\frac{\partial}{\partial t}\left(\begin{array}{l}
I_{0} \\
I_{1} \\
I_{2} \\
I_{3}
\end{array}\right)+\left(\begin{array}{cccc}
0 & c & 0 & 0 \\
c / 3 & 0 & 2 c / 3 & 0 \\
0 & 2 c / 5 & 0 & 3 c / 5 \\
0 & 0 & 3 c / 7 & 0
\end{array}\right) \frac{\partial}{\partial r}\left(\begin{array}{l}
I_{0} \\
I_{1} \\
I_{2} \\
I_{3}
\end{array}\right)+\left(\begin{array}{l}
b_{0} \\
b_{1} \\
b_{2} \\
b_{3}
\end{array}\right)=0
$$

With

$$
A=\left(\begin{array}{cccc}
0 & c & 0 & 0 \\
c / 3 & 0 & 2 c / 3 & 0 \\
0 & 2 c / 5 & 0 & 3 c / 5 \\
0 & 0 & 3 c / 7 & 0
\end{array}\right),
$$

setring

$$
\operatorname{det}|A-\lambda \hat{I}|=0
$$

yields the values for $\lambda$,

$$
\lambda= \pm c\left(\frac{3}{7}+\frac{\alpha}{2}\right)^{1 / 2}, \pm c\left(\frac{3}{7}-\frac{\alpha}{2}\right)^{1 / 2}
$$

where

$$
\alpha^{2}=\frac{96}{245}
$$

Hence, the $\mathrm{P}_{3}$ equations are also hyperbollc. 
In order to use the method of characteristics, the $P_{N}$ equations must be reduced to canonical form. In this formulation, the partial derivative terms in each equation will depend on only one dependent variable. This is accomplished by defining new dependent variables. Examples for reduction of both the $P_{1}$ and $P_{3}$ equations to canonical form follow.

The $P_{1}$ equations can be written as

$$
\frac{\partial I_{0}}{\partial t}+c \frac{\partial I_{I}}{\partial r}+b_{0}=0
$$

and

$$
\frac{\partial I_{1}}{\partial t}+\frac{c}{3} \frac{\partial I_{0}}{\partial r}+b_{1}=0
$$

where

$$
\begin{aligned}
& b_{0}=\frac{2 c}{r} I_{1}(\nu)+c I_{0}(\nu) \sigma_{s}(\nu)-c \int_{0}^{\infty} d \nu^{\prime} \frac{\nu}{\nu^{\prime}} \sigma_{s 0}\left(\nu^{\prime}+\nu\right) I_{0}\left(\nu^{\prime}\right) \\
& -\frac{c^{3}}{8 \pi h}\left\{I_{0} \int_{0}^{\infty} d v^{\prime}\left[\frac{1}{v^{\prime} v^{2}} \sigma_{s 0}\left(v^{\prime}+v\right)-\frac{1}{v^{\prime 3}} \sigma_{s 0}\left(v \rightarrow v^{\prime}\right)\right] I_{0}\left(v^{\prime}\right)\right. \\
& \left.+3 I_{l}(v) \int_{0}^{\infty} d v^{\prime}\left[\frac{1}{v^{\prime} v^{2}} \sigma_{s l}\left(v^{\prime}+v\right)-\frac{1}{v^{\prime 3}} \sigma_{s l}\left(v \rightarrow v^{\prime}\right)\right] I_{1}\left(v^{\prime}\right)\right\}
\end{aligned}
$$

and 


$$
\begin{aligned}
& b_{1}=\frac{c}{3} \sigma_{s}(v) I_{1}(v)-c \int_{0}^{\infty} d v^{\prime} \frac{v}{v^{\prime}} \sigma_{s 1}\left(v^{\prime} \rightarrow v\right) I_{1}\left(v^{\prime}\right) \\
& -\frac{c^{3}}{16 \pi h}\left\{2 I_{0}(v) \int_{0}^{\infty} d v^{\prime}\left[\frac{1}{v^{\prime} v^{2}} \sigma_{s 1}\left(v^{\prime}+v\right)-\frac{1}{v^{\prime 3}} \sigma_{s 1}\left(v+v^{\prime}\right)\right] I_{1}\left(v^{\prime}\right)\right. \\
& \left.+\frac{2}{3} I_{1}(v) \int_{0}^{\infty} d v^{\prime}\left[\frac{1}{v^{\prime} v^{2}} \sigma_{s 0}\left(v^{\prime} \rightarrow v\right)-\frac{1}{v^{\prime 3}} \sigma_{s 0}\left(v+v^{\prime}\right)\right] I_{0}\left(v^{\prime}\right)\right] \cdot \quad(153)
\end{aligned}
$$

In vector form this is

$$
\frac{\partial \underline{u}}{\partial t}+A \frac{\partial \underline{u}}{\partial r}+\underline{b}(r, t, v, \underline{u})=0,
$$

where

$$
\begin{aligned}
& \underline{\underline{u}}=\left(\begin{array}{l}
I_{0} \\
I_{1}
\end{array}\right), \\
& A=\left(\begin{array}{ll}
0 & c \\
c / 3 & 0
\end{array}\right),
\end{aligned}
$$

and

$$
\underline{b}=\left(\begin{array}{l}
b_{0} \\
b_{1}
\end{array}\right) \text {. }
$$

By setting

$$
\operatorname{det}|A-\lambda \hat{I}|=0 \text {, }
$$




$$
\lambda_{1}=\frac{c}{\sqrt{3}}
$$

and

$$
\lambda_{2}=-\frac{c}{\sqrt{3}} .
$$

If we set

$$
\text { A } \mathrm{P}_{i}=\lambda_{i} \mathrm{P}_{i} \quad \mathrm{i}=1,2 \text {, }
$$

it can be found that

$$
E_{I}=\left(\begin{array}{c}
1 \\
\frac{1}{\sqrt{3}}
\end{array}\right)
$$

and

$$
\mathrm{P}_{2}=\left(\begin{array}{l}
1 \\
\frac{1}{\sqrt{3}}
\end{array}\right)
$$

Let ting

$$
P=\left(\begin{array}{rr}
1 & 1 \\
1 & -1 \\
\sqrt{3} & \sqrt{3}
\end{array}\right) \text {, }
$$


we find $t$ iat

$$
p^{-1}=\frac{1}{2}\left(\begin{array}{cc}
1 & \sqrt{3} \\
1 & -\sqrt{3}
\end{array}\right) \text {. }
$$

This allows us to determine two new canonical variables by setcing $\underline{y}=\mathrm{P}^{-1} \underline{u}$. These new variables are

$$
v_{u}=\frac{1}{2}\left[I_{0}+\sqrt{3} I_{1}\right]
$$

and

$$
v_{1}=\frac{1}{2}\left[I_{0}-\sqrt{3} I_{1}\right] .
$$

The matrix $A$, defined as

$$
\therefore=\left(\begin{array}{ll}
\lambda_{1} & 0 \\
0 & \lambda_{2}
\end{array}\right) \text {, }
$$

Decones

$$
A=\left(\begin{array}{cc}
c / \sqrt{3} & 0 \\
0 & -c / \sqrt{3}
\end{array}\right) \text {. }
$$

he can now write the $P_{1}$ equations in canonical form as

$$
\frac{\partial \underline{v}}{\partial \tau}+\Lambda \frac{\partial \underline{v}}{\partial r}+\underline{c}(\tau, v, t, \underline{v})=0,
$$


where

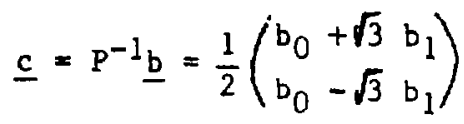

and

$$
\underline{v}=\left(\begin{array}{l}
v_{0} \\
v_{1}
\end{array}\right) \text {. }
$$

Written out, the $P_{1}$ equations are now

$$
\frac{\partial v_{0}}{\partial t}+\lambda_{1} \frac{\partial v_{0}}{\partial r}+c_{0}=0
$$

and

$$
\frac{\partial v_{1}}{\partial t}+\lambda_{2} \frac{\partial v_{1}}{\partial r}+c_{1}=0
$$

where

$$
I_{0}=v_{0}+v_{1}
$$

and

$$
I_{1}=\frac{1}{\sqrt{3}}\left(v_{0}-v_{1}\right) .
$$


In the case of the $\mathrm{P}_{3}$ equations, the reduction to canonical form follows the same procedure. However, it is more complicated. The $\mathrm{P}_{3}$ equations can be written in vector form as

$$
\frac{\partial}{\partial t}\left(\begin{array}{l}
I_{0} \\
I_{1} \\
I_{2} \\
I_{3}
\end{array}\right)+\left(\begin{array}{lccc}
0 & c & 0 & 0 \\
c / 3 & 0 & 2 c / 3 & 0 \\
0 & 2 c / 5 & 0 & 3 c / 5 \\
0 & 0 & 3 c / 7 & 0
\end{array}\right) \frac{\partial}{\partial r}\left(\begin{array}{l}
I_{0} \\
I_{1} \\
I_{2} \\
I_{3}
\end{array}\right)+\left(\begin{array}{l}
b_{0} \\
b_{1} \\
b_{2} \\
b_{3}
\end{array}\right)=0
$$

The expressions for $b_{0}, b_{1}, b_{2}$, and $b_{3}$ can be found by writing out the $P_{3}$ equations in $f_{u l l}$. Equation (177) can be rewritten as

$$
\frac{\partial u}{\partial t}+A \frac{\partial u}{\partial r}+\underline{b}(r, t, v, \underline{u})=0
$$

where

$$
\underline{u}=\left(\begin{array}{l}
I_{0} \\
I_{1} \\
I_{2} \\
I_{3}
\end{array}\right)
$$

$$
A=\left(\begin{array}{cccc}
0 & c & 0 & 0 \\
c / 3 & 0 & 2 c / 3 & 0 \\
0 & 2 c / 5 & 0 & 3 c / 5 \\
0 & 0 & 3 c / 7 & 0
\end{array}\right),
$$

and

$$
\underline{b}=\left(\begin{array}{l}
b_{0} \\
b_{1} \\
b_{2} \\
b_{3}
\end{array}\right)
$$


The eigenvalues, $\lambda_{i}$, were previously found to be

$$
\begin{aligned}
& \lambda_{1}=c\left(\frac{3}{7}+\frac{\alpha}{2}\right)^{1 / 2}, \lambda_{2}=-c\left(\frac{3}{7}+\frac{\alpha}{2}\right)^{1 / 2}, \\
& \lambda_{3}=c\left(\frac{3}{7}-\frac{a}{2}\right)^{1 / 2}, \text { and } \lambda_{4}=-c\left(\frac{3}{7}-\frac{a}{2}\right)^{1 / 2} .
\end{aligned}
$$

If we set

$$
A \underline{p}_{i}=\lambda_{i} \underline{p}_{i}
$$

for $i=1,2,3,4$, the $\underline{p}_{i}$ can be found to be

$$
\underline{p}_{i}=\left(\begin{array}{c}
\frac{35 \lambda_{i}}{6 c}-\frac{3 c}{2 \lambda_{i}} \\
\frac{35 \lambda_{i}^{2}}{6 c^{2}}-\frac{3}{2} \\
\frac{7 \lambda_{i}}{3 c} \\
1
\end{array}\right)
$$

This yields the matrices 


$$
\mathrm{P}^{-1}=\left(\begin{array}{llll}
.053004 & .136931 & .162280 & .113069 \\
-.053004 & .136931 & -.162280 & .113069 \\
-.134253 & -.136931 & .219248 & .386931 \\
.134253 & -.136931 & -.219248 & .386931
\end{array}\right)
$$

and

$$
P=\left(\begin{array}{cccc}
3.281411 & -3.281411 & -2.928788 & 2.428788 \\
2.825742 & 2.825742 & -.825742 & -.825742 \\
2.009318 & -2.009318 & .793289 & -.793289 \\
1.000000 & 1.000000 & 1.000000 & 1.000000
\end{array}\right) \text {. }
$$

The canonical vector form of Eq. (177) is

$$
\frac{\partial \underline{v}}{\partial t}+\Lambda \frac{\partial v}{\partial r}+\underline{c}(r, v, t, \underline{v})=0
$$

where

$$
\Lambda=\left(\begin{array}{cccc}
\lambda_{1} & 0 & 0 & 0 \\
0 & \lambda_{2} & 0 & 0 \\
0 & 0 & \lambda_{3} & 0 \\
0 & 0 & 0 & \lambda_{4}
\end{array}\right)
$$

and $c=P^{-1} \underline{b}$. Writing the $I_{i}$ in terms of the $v_{i}$ yields 


$$
\begin{aligned}
& I_{0}=3.2811411 v_{0}-3.281411 v_{1}-2.128788 v_{2}-2.428788 v_{3},(189) \\
& I_{1}=2.825742 v_{0}-2.825742 v_{1}-.825742 v_{2}-.825742 v_{3}, \quad(190) \\
& I_{2}=2.009318 v_{0}-2.009318 v_{1}+.793289 v_{2}-.793289 v_{3}, \quad(191)
\end{aligned}
$$

and

$$
\mathrm{I}_{3}=\mathrm{v}_{0}+\mathrm{v}_{1}+\mathrm{v}_{2}+\mathrm{v}_{3}
$$

3. 2 General Merhod

For convenience, we rewrite Eqs. (173) and (174) as

$$
\frac{\partial v_{0}}{\partial t}+\lambda_{1} \frac{\partial v_{0}}{\partial r}+c_{0}=0
$$

and

$$
\frac{\partial v_{1}}{\partial t}+\lambda_{2} \frac{\partial v_{1}}{\partial r}+c_{1}=0,
$$

where

$$
c_{0}=\frac{1}{2} b_{0}+\frac{\sqrt{3}}{2} b_{1}
$$

and 


$$
c_{1}=\frac{1}{2} b_{0}-\frac{\sqrt{3}}{2} b_{1}
$$

Here, $b_{0}$ and $b_{1}$ are given by Eqs. (155) and (156). We can rewrite Eqs. (193) and (194) as

$$
\frac{d v_{0}}{d t}+c_{0}=0
$$

and

$$
\frac{d v_{1}}{d t}+c_{1}=0
$$

where

$$
\frac{d v_{0}}{d t}=\left(\frac{\partial}{\partial t}+\lambda_{1} \frac{\partial}{\partial r}\right) v_{0}
$$

and

$$
\frac{d v_{1}}{d t}=\left(\frac{\partial}{\partial t}+\lambda_{2} \frac{\partial}{\partial r}\right) v_{1}
$$

This gives expressions for the total time derivatives of $v_{0}$ and $v_{1}$ along the curves defined by

$$
\frac{d r}{d \tau}=\lambda_{1}
$$


and

$$
\frac{d r}{d t}=\lambda_{2}
$$

respectively. These curves are known as the characteristic curves of Eqs. (193) and (194). Solutions to Eqs. (193) and (194) can be found at points where two characteristic curves intersect. The families of characteristic curves can be found by integrating Eqs. (201) and (202) to obtain

$$
r=\lambda_{1} t+\text { constant }
$$

and

$$
r=\lambda_{2} t+\operatorname{constant}
$$

The solution to Eqs. (193) and (194) is found in the following manner. We assume an initial condition is known at time $t_{0}$. We use the values of $v_{0}$ and $v_{1}$ at point $A$ (Figure 3 ) to find a value of $v_{0}$ at point D. The values of $v_{0}$ and $v_{1}$ at point $B$ are used to find a value of $v_{1}$ at point $D$. The same is done at points $B$ and $C$ to obtain values of $v_{0}$ and $v_{1}$ at point $E$. Then, this procedure is used at points $D$ and $E$ to obtain $v_{0}$ and $v_{1}$ at point $F$. This process is used 


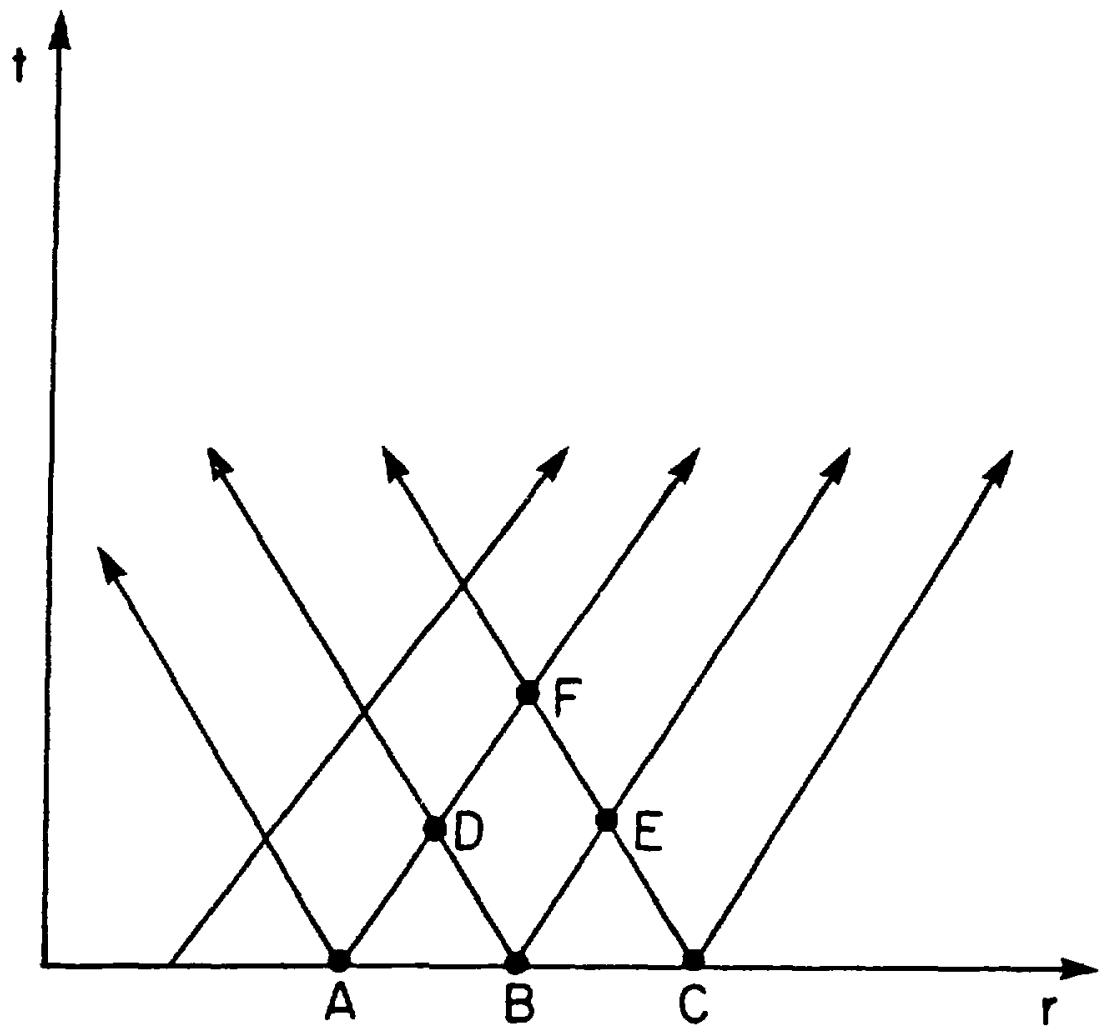

Figure 3.

Geometry for two families of characteristic curves. 
to propagate a solution forward in time from knowledge of the initial conditions.

From Eqs. (193) and (194) we can write

$$
v_{i}(t)=v_{i}\left(t_{0}\right)-\int_{t_{0}}^{t} d t^{\prime} C_{i}\left(r\left(\lambda_{i}, t^{\prime}\right), t^{\prime}, v_{0}\left(t^{\prime}\right), v_{l}\left(t^{\prime}\right)\right)
$$

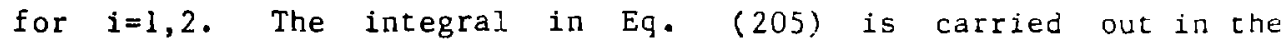
following manner.

Let

$$
\int_{t_{0}}^{t} d t^{\prime} C_{i}=C_{i}\left(r\left(\lambda_{i}, t_{0}\right), t_{0}, v_{0}\left(t_{0}\right), v_{1}\left(t_{0}\right)\right)\left(t-t_{0}\right)
$$

for $1=1,2$. This gives a first estimate for $v_{0}(t)$ and $v_{1}(t)$ say, $v_{0}^{\prime}(t)$ and $v_{i}^{\prime}(t)$. We now resolve for $v_{0}(t)$ and $v_{1}(t)$ by setcing

$$
\begin{aligned}
& \int_{t_{0}}^{t} d t^{\prime} C_{i}=\frac{1}{2}\left[C_{i}\left(r\left(\lambda_{i}, t\right), t, v_{0}^{\prime}(t), v_{1}^{\prime}(t)\right)\right. \\
& \left.\quad+C_{i}\left(r\left(\lambda_{i}, t_{0}\right), t_{0}, v_{0}\left(t_{0}\right), v_{1}\left(t_{0}\right)\right)\right]\left(t-t_{0}\right) .
\end{aligned}
$$

This yields a second order estimate of $v_{0}(t)$ and $v_{1}(t)$. This new estimate is then reentered into Eq. (207) to obtain an even better estimate for $v_{0}(t)$ and $v_{1}(t)$. This method of successive approximations converges quite rapidly. In the problems addressed in this thesis, only five or less iterations were required to yield good convergence. One point of importance in this method is the size of the time step used. If the time step is too large and a rapid 
variation occurs in $v_{0}$ or $v_{1}$ over that time interval, the method diverges quite rapidly.

In the $\mathrm{P}_{3}$ approximation, a set of four equations must be solved. The same method as that described above can be used to solve the problem along four characteristic curves. However, construction of the gridwork necessary to propagate a solution upward in time is not as simple as in the $P_{1}$ case. An example of the grid necessary to solve a $P_{3}$ problem where a source is turned on at $t=0$ located at $r=0$ is illustrated in Fig. (4). The values are known at points beyond which the radiation can propagate unimpeded. Hence, no gridwork is required beyond those points.

An error is introduced by the $\mathrm{P}_{N}$ approximation which becomes acutely apparent when using the method of characteristics. The maximum propagation velocity of the radiation is given by the largest eigenvalue used to compute the characteristic curves. In the $\mathrm{P}_{1}$ approximation, this speed is $.577 c$. In the $\mathrm{P}_{2}$ approximation, this becomes $.775 \mathrm{c}$. In the $P_{3}$ approximation, the maximum velocity is .861c. This velocity approaches the speed of light in a vacuum as the order of the approximation 1ncreases.

The process for handling the frequency integrals is quite simple. We solve for the values of $v_{0}$ and $v_{1}$ only at specific frequency points, say at all $v_{i}$ for $i=1,2, \ldots, G$. The variables $v_{0}$ and $v_{1}$ are assumed to be linear between adjacent frequency points. Each step used to calculate $v_{0}$ and $v_{1}$, described by Eqs. (206) and (207), is performed at each ir requency point. The integrals over frequency space are performed using the trapezoid rule for numerical 


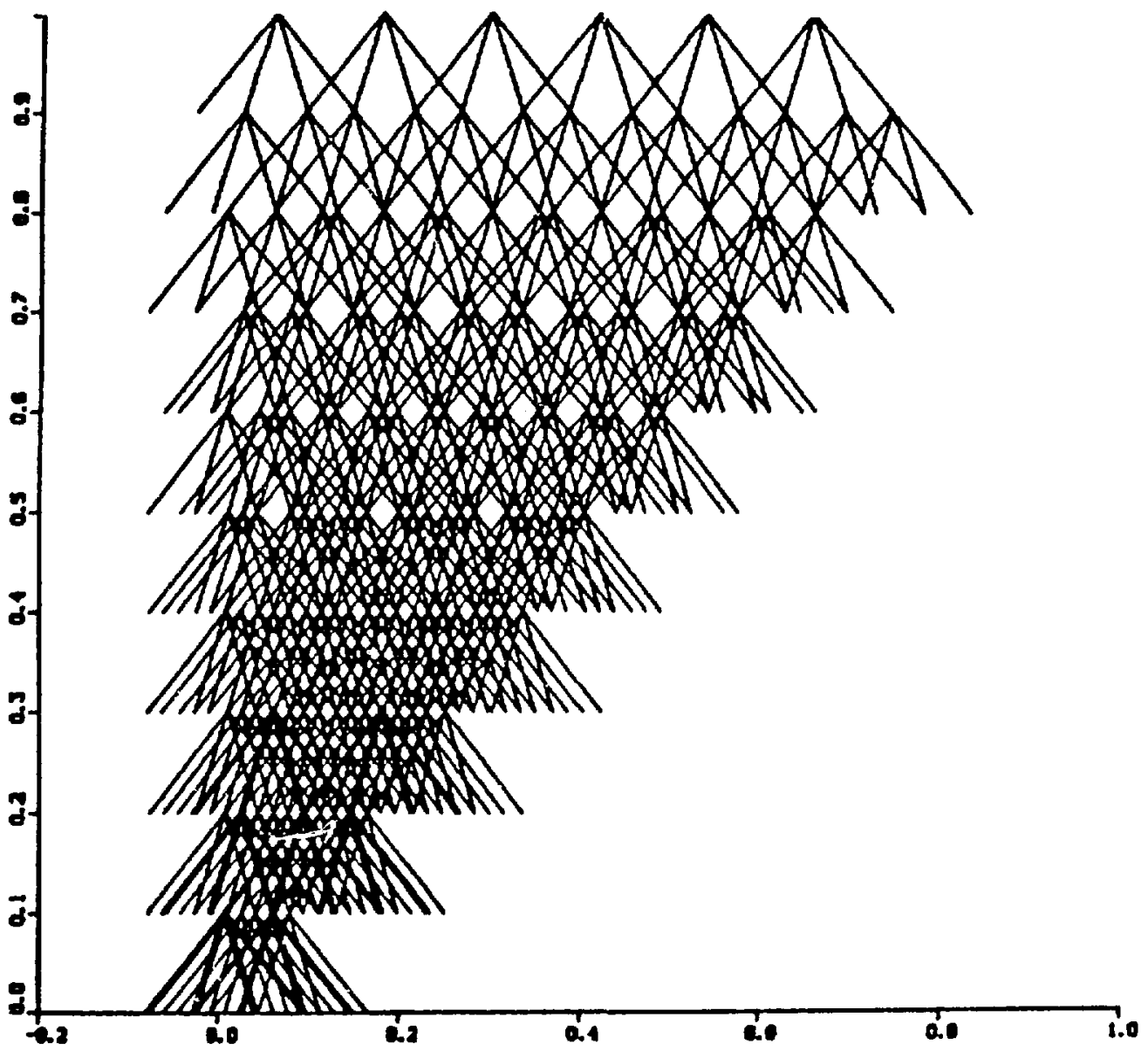

$$
\text { Figure } 4 .
$$

Characteristic curves for a $P_{3}$ problem. 
integration. This method is discussed more fully in Appendix II.

3.3 Evaluation of the Scattering Kernel

In order to write down the actual equations to be solved in this thesis, more must be known concerning the scattering cross sections to be used. In this section, the derivation of the moments of the scattering kernel for two cases is given, one in which the electrons are assumed fixed, the other in which the electrons are described by a relativistic Maxwellian distribution. Actual solutions were found to problems in which the electrons were modeled with the relativistic Maxwellian.

Scattering of photons by free electrons at rest can be modeled using the Klein-Nishina formula, namely, $(17,26)$

$$
\begin{aligned}
\sigma_{\mathrm{KN}}\left(\nu+\nu^{\prime}, \underline{\Omega} \cdot \underline{\Omega}^{\prime}\right)= & \frac{\mathrm{r}_{0}^{2}}{2 \gamma^{\nu}}\left[1+\mu^{2}+\gamma \gamma^{\prime}(1-\mu)^{2}\right] \\
& \times \delta\left(\mu-1+\frac{1}{\gamma^{\prime}}-\frac{1}{\gamma}\right),
\end{aligned}
$$

where

$$
\mu=\underline{\Omega} \cdot \underline{\Omega^{\prime}}
$$

and 


$$
\gamma=\frac{h v}{m_{0} c^{2}}
$$

The quantity

$$
r_{0}=\frac{e^{2}}{m_{e} c^{2}}
$$

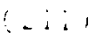

is the classical electron radius.

If we write the scatcering kernel as the inilite series

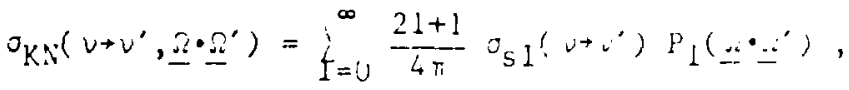

we can find the expansion coefticients $\sigma_{s j}\left(v+v^{\prime}\right)$ and $o_{s j}\left(\rightarrow v^{\prime}\right)$. This is done ty noting that

$$
\int_{-1}^{1} \mathrm{~d} u P_{n}\left(\underline{\Omega}^{\prime} \cdot \underline{\Omega}\right) \sigma_{K N}\left(v+v^{\prime}, \underline{\Omega} \cdot \underline{\Omega}\right)=\frac{1}{2 \pi} \sigma_{s n}\left(v+v^{\prime}\right) \text {. }
$$

By substituting Eq. (208) into Eq. (213) and carrying out the integration for $n=0$ and $n=1$, the expansion coefficients can be obtained. They are

$$
\sigma_{s 0}\left(\nu^{\prime} \rightarrow \nu\right)=\frac{\pi r_{0}^{2}}{\gamma^{\prime} \nu^{\prime}}\left[\frac{\gamma^{\prime}-2}{\gamma}+\frac{\gamma+2}{\gamma^{\prime}}+\left(\frac{1}{\gamma^{\prime}}-\frac{1}{\gamma}\right)^{2}\right]
$$


and

$$
a_{s 1}\left(v^{\prime}+v\right)=\frac{\pi r_{0}^{2}}{r^{\prime} v^{\prime}}\left(1-\frac{1}{r}+\frac{1}{r^{\prime}}\right)\left(\frac{r^{\prime}-2}{r}+\frac{\gamma+2}{r^{\prime}}+\left(\frac{1}{r^{\prime}}-\frac{1}{r}\right)^{2}\right)
$$

The total scattering cross section is (23)

$$
\begin{gathered}
a_{S}(\nu)=2 \pi r_{0}^{2}\left\{\frac{1+\gamma}{\gamma^{3}}\left\{\frac{2 \gamma(1+Y)}{1+2 \gamma}-\log (1+2 \gamma)\right\}\right. \\
\left.+\frac{1}{2 \gamma} \log (1+2 \gamma)-\frac{1+3 \gamma}{(1+2 \gamma)^{2}}\right\} .
\end{gathered}
$$

It should be noted that the above equations are the microscopic cross sections. These can be converted to the nacruscopic cross sections by simply wultiplying by the electron number density $\mathrm{n}_{\mathrm{e}}$, through which spatial dependence can be introduced into the scattering kernel.

If we assume that the electrons have zero kinetic energy so that no upscatcering occurs, the Compton formula for the maximum possible frequency shift, where $v_{i}$ is the initial frequency, is

$$
v=\frac{v_{i}}{1+\frac{h v_{1}}{m_{e} c^{2}}}
$$

For the outscattering terms of the transport equation the lower limit of integration is 


$$
\frac{v}{1+\frac{h v}{m_{e} c^{2}}}
$$

and the upper limit is simply $v$. For inscattering terms the lower limit is $v$ and the upper limit of integration is

$$
\frac{v}{1-\frac{h v}{m_{e^{c}} c^{2}}} \text {. }
$$

In the problems addressed in this thesis, the electrons are in mrtion. Hence, the scattering terms already discussed must be altered to take this into account. It is assumed that the electron velocity distribution can be modeled by a relativistic Maxiellian distribution. The normalized distribution is given by $(18,20)$

$$
f(\underline{v})=\frac{\mathbb{m}_{0} \lambda^{5} \exp \left(-m_{0} c^{2} \dot{\lambda} / k T\right)}{4 \pi c k T k_{2}\left(m_{0} c^{2} / k T\right)} .
$$

Here, $\mathrm{K}_{2}$ is the modified Bessel function of second order. Also,

$$
\lambda=\left(1-\frac{v^{2}}{c^{2}}\right)^{-1 / 2},
$$


where $v=|\underline{v}|$ and $k T$ is the temperature of the electrons. The energy density of the electrons can be calculated from

$$
\iint_{0}^{\infty} d^{3} v \frac{1}{2} m_{0} \lambda v^{2} f(\underline{v}) n_{e}
$$

where $n_{e}$ is the electron density.

Substituting Eq. (220) into Eq. (222) and carrying out the integration yields the energy density, $u_{e}$, as

$$
u_{e}=n_{e} \frac{3}{2} k T
$$

The Klein-ivishina formula for the scattering kernel,

$$
\begin{aligned}
& \sigma_{K N}\left(\nu+\nu^{\prime}, \Omega \cdot \Omega^{\prime}\right)=\frac{r_{0}^{2}}{2 \gamma v}\left[1+\mu^{2}+\gamma \gamma^{\prime}(1-\mu)^{2}\right] \\
& \times \delta\left(\mu-1+\frac{1}{Y^{\prime}}-\frac{1}{\gamma}\right),
\end{aligned}
$$

is for the reference frame in which the electrons are at rest. The transport equation being used, however, applies in the frame in which the observer is at rest. Since we are considering the case in which the electrons are in motion, the scattering cross sections must be Lorentz transformed. If we denote quantities in the electron rest frame with a zero subscript, and quantities in the observer rest frame are unadorned, we have 


$$
v_{0}=\lambda D v
$$

and

$$
\nu_{0}^{\prime}=\lambda D^{\prime} v^{\prime},
$$

where

$$
\begin{aligned}
& \lambda=\left(1-\frac{v^{2}}{c^{2}}\right)^{-1 / 2,}, \\
& D=1-\underline{\Omega} \cdot \underline{v} / c, \\
& D^{\prime}=1-\underline{\Omega} \cdot \underline{v} / c,
\end{aligned}
$$

and

$$
1-\Omega_{0} \cdot \Omega_{0}^{\prime}=\frac{1-\underline{\Omega} \cdot \underline{\Omega}^{\prime}}{\lambda^{2} \mathrm{DD}^{\prime}} \text {. }
$$

This allows us to write the Lorentz transformed scattering kernel as

$$
\sigma_{s}\left(v+v^{\prime}, \underline{\Omega} \cdot \underline{\Omega}^{\prime}\right)=\sigma_{K N}\left(v_{0}+v_{0}^{\prime}, \underline{\Omega}_{0} \cdot \underline{\Omega}_{0}^{\prime}\right) \frac{\mathrm{D}}{\mathrm{D}^{\prime}} .
$$

This gives the scattering kernel for a specific electron velocity. In order to arrive at a form which is useful, this 
expression must be averaged over the velocity distribution of the electrons. If this distribution is given by $\mathrm{f}(\underline{\mathrm{v}})$, we have

$$
\sigma_{s}\left(v+v^{\prime}, \underline{\Omega^{\prime}} \cdot \underline{\Omega}^{\prime}, T\right)=\int \underline{d \underline{v}} f(\underline{v}) \sigma_{K \Lambda^{\prime}}\left(v_{0}+v_{0}^{\prime}, \Omega_{0} \cdot \Omega_{0}^{\prime}\right) \frac{D}{\lambda D^{\prime}} .
$$

The expansion coefficients for the Legendre expansion of the scattering kernel are found numerically from

$$
\begin{aligned}
\sigma_{S U}\left(r, v \rightarrow v^{\prime}\right) & =2 \pi \int_{-1}^{1} d \mu \delta d \underline{v} f(\underline{v}) \sigma_{K N}\left(\nu_{0} \rightarrow v_{0}^{\prime}, \Omega_{0} \cdot \Omega_{0}^{\prime}\right) \\
\times & \frac{D}{\lambda D^{\prime}} P_{0}(\mu)
\end{aligned}
$$

and

$$
\begin{gathered}
\sigma_{\mathrm{SI}}\left(r, v \rightarrow v^{\prime}\right)=2 \pi \int_{-1}^{1} \mathrm{~d} \mu \delta \mathrm{d} \underline{\underline{v}} \mathrm{f}(\underline{v}) \sigma_{\mathrm{KN}}\left(\nu_{0} \rightarrow v_{0}^{\prime}, \Omega_{0} \cdot \underline{\Omega}_{0}^{\prime}\right) \\
\times \frac{\mathrm{D}}{\lambda D^{\prime}} P_{1}(\mu) .
\end{gathered}
$$

Also,

$$
\begin{aligned}
& \sigma_{s}(v)=2 \pi \int_{0}^{\infty} d v^{\prime} \int_{-1}^{1} d \mu P_{0}(\mu) \int d \underline{v} E(\underline{v}) \sigma_{K N}\left(v_{0} \rightarrow v_{0}^{\prime}, \Omega_{0} \cdot \Omega_{0}^{\prime}\right) \frac{D}{\lambda D^{\prime}} \\
& =\int_{0}^{\infty} d v^{\prime} \sigma_{s}\left(r, v \rightarrow v^{\prime}\right)
\end{aligned}
$$

The evaluation of these equations for the scattering coefficlents is obtained with the code developed by Gerald Minerbo. (44) 
The detailed balance condition is to be used, and the scattering kernel averaged over a relativistic Maxwellian distribution. Consequently, we have assumed that during the evolution of the radiation frequency profile, the electron field must remain in equilibrium with itself. In a plasma, the electron-electron collision frequency can be written as (24)

$$
\nu_{e e}=\frac{3 \times 10^{-6} n \ln \Lambda}{T_{e V} 3 / 2} \text {, }
$$

where $n$ is the electron density, $T_{e V}$ is the electron temperature in $\mathrm{eV}$, and a typical value for $\ln \Lambda$ is twenty.(24) For $\mathrm{n}=10^{20}$ and $\mathrm{T}_{\mathrm{eV}}=4000 \mathrm{eV}$, the collision time, $1 / \nu_{\mathrm{ee}}$, is $10^{-11}$ seconds. An upper bound on the photon-electron collision time can be found by using a maximum collision cross section of $\pi r_{U}^{2}$ where $r_{0}$ is the classical electron radius. A collision frequency can be obtained from $\pi r_{0}{ }^{2} \mathrm{nc}$ which, for the above density, gives a collision time of $10^{-6}$ seconds. This is a five order of magnitude difference. Hence, it appears our assumption is qujte reasonable.

The canonical form of the equations we wish to solve are

$$
\frac{\partial v_{0}}{\partial t}+\lambda_{1} \frac{\partial v_{0}}{\partial r}+c_{0}=0
$$

and 


$$
\frac{\partial v_{1}}{\partial t}+\lambda_{2} \frac{\partial v_{1}}{\partial r}+c_{1}=0
$$

where

$$
I_{0}(r, v, t)=v_{0}(r, v, t)+v_{1}(r, v, t)
$$

and

$$
I_{1}(r, \nu, t)=\frac{1}{\sqrt{3}}\left[v_{0}(r, \nu, t)-v_{1}(r, \nu, t)\right] .
$$

The expressions for $c_{0}$ and $c_{1}$ are

$$
\begin{aligned}
& c_{0 / 1}=\frac{c}{r} I_{1}+\frac{c}{2} I_{0} \sigma_{s}(\nu)-\frac{c}{2} \int_{0}^{\infty} d v^{\prime} \frac{\nu}{\nu^{\prime}} \sigma_{s 0}\left(\nu^{\prime}+\nu\right) I_{0}\left(\nu^{\prime}\right) \\
& -\frac{c^{3}}{16 \pi}+I_{0} \int_{0}^{\infty} 1 \frac{1}{v^{\prime} v^{2}} \sigma_{s 0}\left(v^{\prime} \rightarrow v\right) \\
& \left.-\frac{1}{v^{\prime}} \sigma_{s 0}\left(v \rightarrow v^{\prime}\right)\right\rfloor I_{0}\left(v^{\prime}\right) \\
& +3 I_{1} \int_{0}^{\infty} \mathrm{d} \nu^{\prime}\left[\frac{1}{v^{\prime} v^{2}} \sigma_{\mathrm{s} 1}\left(\nu^{\prime}+\nu\right)\right. \\
& \left.\left.-\frac{1}{v^{\prime} 3} \sigma_{s l}\left(v+v^{\prime}\right)\right] I_{1}\left(v^{\prime}\right)\right\} \\
& \pm \frac{\sqrt{3} c}{6} \sigma_{s}(v) I_{1} \mp \frac{\sqrt{3} c}{2} \int_{0}^{\infty} \frac{\nu}{v^{\prime}} \sigma_{s 1}\left(v^{\prime}+v\right) I_{1}\left(v^{\prime}\right) \\
& \mp \frac{\sqrt{3} c^{3}}{16 \pi h}\left\{I_{0} \int_{0}^{\infty} \mathrm{d} \nu^{\prime}\left[\frac{1}{v^{\prime} v^{2}} \sigma_{s 1}\left(v^{\prime}+v\right)-\frac{1}{v^{\prime}} \sigma_{s 1}\left(v \rightarrow v^{\prime}\right)\right] I_{1}\left(v^{\prime}\right)\right.
\end{aligned}
$$

88 


$$
\begin{aligned}
+\frac{1}{3} I_{1} & \int_{0}^{\infty} d v^{\prime}\left\{\frac{1}{v^{\prime} v^{2}} \sigma_{s}\left(v^{\prime}+v\right)\right. \\
& \left.\left.-\frac{1}{v^{\prime 3}} \sigma_{s 0}\left(v+v^{\prime}\right)\right] I_{0}\left(v^{\prime}\right)\right\} .
\end{aligned}
$$

In the above equation, $c_{0}$ is found by using the upper sign in the terms with the symbol \pm or $\mp$, and $c_{1}$ is found by using the lower sign.

By combining terms and substituting expressions for $I_{0}$ and $I_{1}$ in terms of $v_{0}$ and $v_{1}$ into Eq. (240), we can rewrite Eq. (240) as

$$
\begin{aligned}
& c_{0 / 1}=\frac{c}{\sqrt{3} r}\left(v_{0}-v_{1}\right)+\frac{c}{2} \sigma_{s}(v)\left[v_{0}+v_{1} \pm \frac{1}{3}\left(v_{0}-v_{1}\right)\right] \\
& -\frac{c}{2} \int_{0}^{\infty} d v^{\prime} \frac{v}{v^{\prime}}\left[\sigma_{s}\left(v^{\prime} \rightarrow v\right)\left(v_{0}\left(v^{\prime}\right)+v_{1}\left(v^{\prime}\right)\right)\right. \\
& \left.\quad \pm \sigma_{s 1}\left(v^{\prime}+v\right)\left(v_{0}\left(v^{\prime}\right)-v_{1}\left(v^{\prime}\right)\right)\right] \\
& -\frac{c^{3}}{16 \pi h}\left\{1 v_{0}+v_{1} \pm \frac{1}{3}\left(v_{0}-v_{1}\right)\right] \int_{0}^{\infty}\left[\frac{1}{v^{\prime} v^{2}} \sigma_{s 0}\left(v^{\prime} \rightarrow v\right)\right. \\
& \left.\left.-\frac{1}{v^{\prime}} \sigma_{s 0^{\prime}}\left(v \rightarrow v^{\prime}\right)\right]\left(v_{0}\left(v^{\prime}\right)+v_{1}\left(v^{\prime}\right)\right)+1 \sqrt{3}\left(v_{0}-v_{1}\right) \pm\left(v_{0}+v_{1}\right)\right] \\
& \times \int_{0}^{\infty} d v^{\prime}\left[\frac{1}{v^{\prime} v^{2}} \sigma_{s 1}\left(v^{\prime} \rightarrow v\right)-\frac{1}{v^{3}} \sigma_{s 1}\left(v+v^{\prime}\right)\right] \\
& \left.\times \frac{1}{\sqrt{3}}\left(v_{0}\left(v^{\prime}\right)-v_{1}\left(v^{\prime}\right)\right\}\right)(241)
\end{aligned}
$$

This gives the formulation of the $P_{1}$ radiation transport problem as needed for use with the method of characteristics. It contains Induced scattering effects but does not contain absorption or 
emission. Inclusion of these effects is easily accomplished by adding several terms to the expressions for $c_{0}$ and $c_{1}$. Inclusion of these terms yields the expressions

$$
\begin{aligned}
& c_{0 / 1}=\frac{c}{\sqrt{3} r}\left(v_{0}-v_{1}\right)+\frac{1}{2} \sigma_{a}^{\prime}(v)\left[v_{0}+v_{1}-4 \pi B(v) \pm c\left(v_{0}-v_{1}\right)\right] \\
& +\frac{c}{2} o_{5}(v)\left[v_{0}+v_{1} \pm \frac{1}{3}\left(v_{0}-v_{1}\right)\right] \\
& -\frac{c}{2} \int_{0}^{\infty} d v^{\prime} \frac{v}{v^{\prime}}\left\{\sigma_{s 0}\left(v^{\prime}+v\right)\left(v_{0}\left(v^{\prime}\right)+v_{1}\left(v^{\prime}\right)\right)\right. \\
& \left. \pm \sigma_{s 1}\left(v^{\prime}+v\right)\left(v_{0}\left(v^{\prime}\right)-v_{1}\left(v^{\prime}\right)\right)\right] \\
& -\frac{c^{3}}{16 \pi h}\left\{[ v _ { 0 } + v _ { 1 } \pm \frac { 1 } { 3 } ( v _ { 0 } - v _ { 1 } ) ] \int _ { 0 } ^ { \infty } \left[\frac{1}{v^{\prime} v^{2}} \sigma_{s 0}\left(v^{\prime}+v\right)\right.\right. \\
& \left.-\frac{1}{v^{\prime} 3} \sigma_{s 0}\left(v+v^{\prime}\right)\right]\left(v_{0}\left(v^{\prime}\right)+v_{1}\left(v^{\prime}\right)\right)+\left[\sqrt{3}\left(v_{0}-v_{1}\right) \pm\left(v_{0}+v_{1}\right)\right] \\
& x \int_{0}^{\infty} d v^{\prime}\left[\frac{1}{v^{\prime} v^{2}} \sigma_{s 1}\left(v^{\prime}+v\right)-\frac{1}{v^{3}} \sigma_{s 1}\left(v+v^{\prime}\right)\right] \\
& \left.\times \frac{1}{\sqrt{3}}\left(v_{0}\left(v^{\prime}\right)-v_{1}\left(v^{\prime}\right)\right)\right\} .
\end{aligned}
$$

Equations (237) and (238) along with the above expression for $c_{0}$ and $c_{1}$ are the canonical form of the $P_{1}$ radiation transport equations including induced effects, absorption and emission, general scattering, and an arbitrary source. One-dimensional spatial dependence and time dependence is included. Actual solutions are found to problems in which Eq. (241) is used for $c_{0}$ and $c_{1}$. These solutions utiilze the numerical methods discussed in this chapter. 


\section{NUMERICAL RESULTS FOR SPATIALLY DEPENDENT AND SPATIALLY INDEPENDENT RADIATION TRANSPORT PROBLEMS}

All results given in this chapter address the $P_{1}$ radiation transport equations as derived in Chapter Three. The equations are solved in canonical form along their characteristic curves. These equations are written in their canonical form here as

$$
\begin{aligned}
& \frac{\partial v_{0}}{\partial t}+\lambda_{1} \frac{\partial v_{0}}{\partial r}+\frac{c}{\sqrt{3} r}\left(v_{0}-v_{1}\right)+\frac{c}{2} \sigma_{s}(v)\left[\frac{4}{3} v_{0}+\frac{2}{3} v_{1}\right] \\
& -\frac{c}{2} \int_{0}^{\infty} d v^{\prime} \frac{v}{v^{\prime}}\left[\sigma_{s 0}\left(v^{\prime}+v\right)\left(v_{0}\left(v^{\prime}\right)+v_{1}\left(v^{\prime}\right)\right)\right. \\
& \left.\quad+\sigma_{s 1}\left(v^{\prime}+v\right)\left(v_{0}\left(v^{\prime}\right)-v_{1}\left(v^{\prime}\right)\right)\right] \\
& -\frac{c^{\prime}}{15 \pi h}\left\{[ \frac { 4 } { 3 } v _ { 0 } + \frac { 2 } { 3 } v _ { 1 } ] \int _ { C } ^ { \infty } d v ^ { \prime } \left[\frac{1}{v^{\prime} v^{2}} \sigma_{s 0}\left(v^{\prime}+v\right)\right.\right. \\
& \left.-\frac{1}{v^{\prime 3}} \sigma_{s 0}\left(v+v^{\prime}\right)\right]\left(v_{0}\left(v^{\prime}\right)+v_{1}\left(v^{\prime}\right)\right)+\left[\sqrt{3}\left(v_{0}-v_{1}\right)+\left(v_{0}+v_{1}\right)\right. \\
& \times \int_{0}^{\infty} d v^{\prime}\left[\frac{1}{v^{\prime} v^{2}} \sigma_{s l}\left(v^{\prime}+v\right)-\frac{1}{v^{\prime 3}} \sigma_{s 1}\left(v+v^{\prime}\right)\right] \frac{1}{\sqrt{3}} \\
& \left.\times\left(v_{0}\left(v^{\prime}\right)-v_{1}\left(v^{\prime}\right)\right)\right\}=0
\end{aligned}
$$


and

$$
\begin{aligned}
& \frac{\partial v_{1}}{\partial t}+\lambda_{2} \frac{\partial v_{1}}{\partial r}+\frac{c}{\sqrt{3} r}\left(v_{0}-v_{1}\right)+\frac{c}{2} \sigma_{s}(v)\left[\frac{2}{3} v_{0}+\frac{4}{3} v_{1}\right] \\
& -\frac{c}{2} \int_{0}^{\infty} d v^{\prime} \frac{v}{v^{\prime}}\left[\sigma_{s v^{\prime}}\left(v^{\prime}+v\right)\left(v_{0}\left(v^{\prime}\right)+v_{1}\left(v^{\prime}\right)\right)\right. \\
& \left.-\sigma_{s l}\left(v^{\prime} \rightarrow v\right)\left(v_{0}\left(v^{\prime}\right)-v_{1}\left(v^{\prime}\right)\right)\right] \\
& -\frac{c^{3}}{15 \pi h}\left\{[ \frac { 2 } { 3 } v _ { 0 } + \frac { 4 } { 3 } v _ { 1 } \} \int _ { 0 } ^ { \infty } d v ^ { \prime } \left[\frac{1}{v^{\prime} v^{2}} \sigma_{s 0}\left(v^{\prime}+v\right)\right.\right. \\
& \left.-\frac{1}{v^{3}} \sigma_{s 0}\left(v \rightarrow v^{\prime}\right)\right]\left(v_{0}\left(v^{\prime}\right)+v_{1}\left(v^{\prime}\right)\right)+\left[\sqrt{3}\left(v_{0}-v_{1}\right)-\left(v_{0}+v_{1}\right)\right. \\
& x \int_{0}^{\infty} \mathrm{d} v^{\prime}\left[\frac{1}{v^{\prime} v^{2}} \sigma_{s 1}\left(v^{\prime}+v\right)-\frac{1}{v^{\prime}} \sigma_{s 1}\left(v+v^{\prime}\right)\right] \frac{1}{\sqrt{3}} \\
& \left.\times\left(v_{0}\left(v^{\prime}\right)-v_{1}\left(v^{\prime}\right)\right)\right\}=0 \text {. }
\end{aligned}
$$

In Eqs. (243) and (244), $\lambda_{1}$ and $\lambda_{2}$ are given by Eqs. (201) and (202), $v_{0}$ and $v_{1}$ are given by Eqs. (166) and (167), and the scattering cross sections, $\sigma_{S}, \sigma_{s 0}$, and $\sigma_{s l}$, are found from Eqs. $(233)-(235)$

The above equations were solved for a variety of initial conditions. These include blackbody initial spectra and initial spectra containing rectangular spectral features. The literature found on induced Compton effects notes that the evolution of the spectral profile is 'pendent upon the initial profile. $(13,14,15,25)$ No universal beharior is noted. 
In all of the problems solved in this thesis, the frequency points were chosen with a geometric spacing. In most cases, 135 frequency points were used. Problems with up to 200 points were run to test the sensitivity of the results on the number of points used. For the cases with 135 points, the radiation energy had a lower limit of $5 \mathrm{eV}$ and an upper limit of $400 \mathrm{keV}$. Each step was incremented by a factor of 1.087. These limits were chosen to encompass the energy range of interest in small fusion target problems.

A numerical problem was encouncered at the lower frequency end of the multigroup structure. A numerical instability occurred in some problems at the two lowest frequency points. This inscability occurs because no downscatering can occur below the lowest frequency point numerically. Because the intensity is small at these poirits un all of the problems considered, no significant upscattering occurs. Problems were terminated before the instability contributes to the solution. These points are ignored in the graphics output,

\subsection{Spatialiy Independent Solutions}

In these problems, the initial distribution used is spatially homogeneous. This homogeneity remains throughout the duration of tie problem. Consequently, only several points need be calculated after each time step. This solution is now the same for all points at this time. This eliminates the need to compute the solutions at each. spatial point. Consequently, this sharply reduces the computing time required for each problem. 
Several consistency checks are available to help determine the validity of the codes developed. The problems run must conserve photons. This is due to the fact that no absorption or emission is included. An equilibrium distribution at the same temperature as the electrons must remain in equilibrium. Also, initial distributions slightly out of equilibrium must equilibrate with the electrons.

Figure (5) shows the superimposed curves for 100 time steps of an equilibrium distribution. The initial distribution is that of a blackbody at 4kev. The electron field is also at $4 \mathrm{keV}$. The sinall amount of movement in the curve is due to the lower frequency end effects described on Page 92. Because scattering below 5eV is not allowed in the code developed, this error is largest at the low frequency end of the blackbody. After $4 \times 10^{-5}$ seconds, this error was $\sim 25 \%$ at $\sim 15 \mathrm{eV}$. At the peak of the blackbody distribution, this error was negligible.

Another type of problem used to test the code involved rectangular spectral features represented by step functions in frequency space. The initial features were such that interesting induced effects would not occur. Consequently, we expect the profiles to approach that of a blackbody equilibrium distribution. Figure (6) shows a single Initial feature. This is the rectangular feature on which are superimposed curves at later times. The discribution is approaching that of a blackbody. This is the steady state equilibrium distribution. 
INTENSITY VS. FREO.

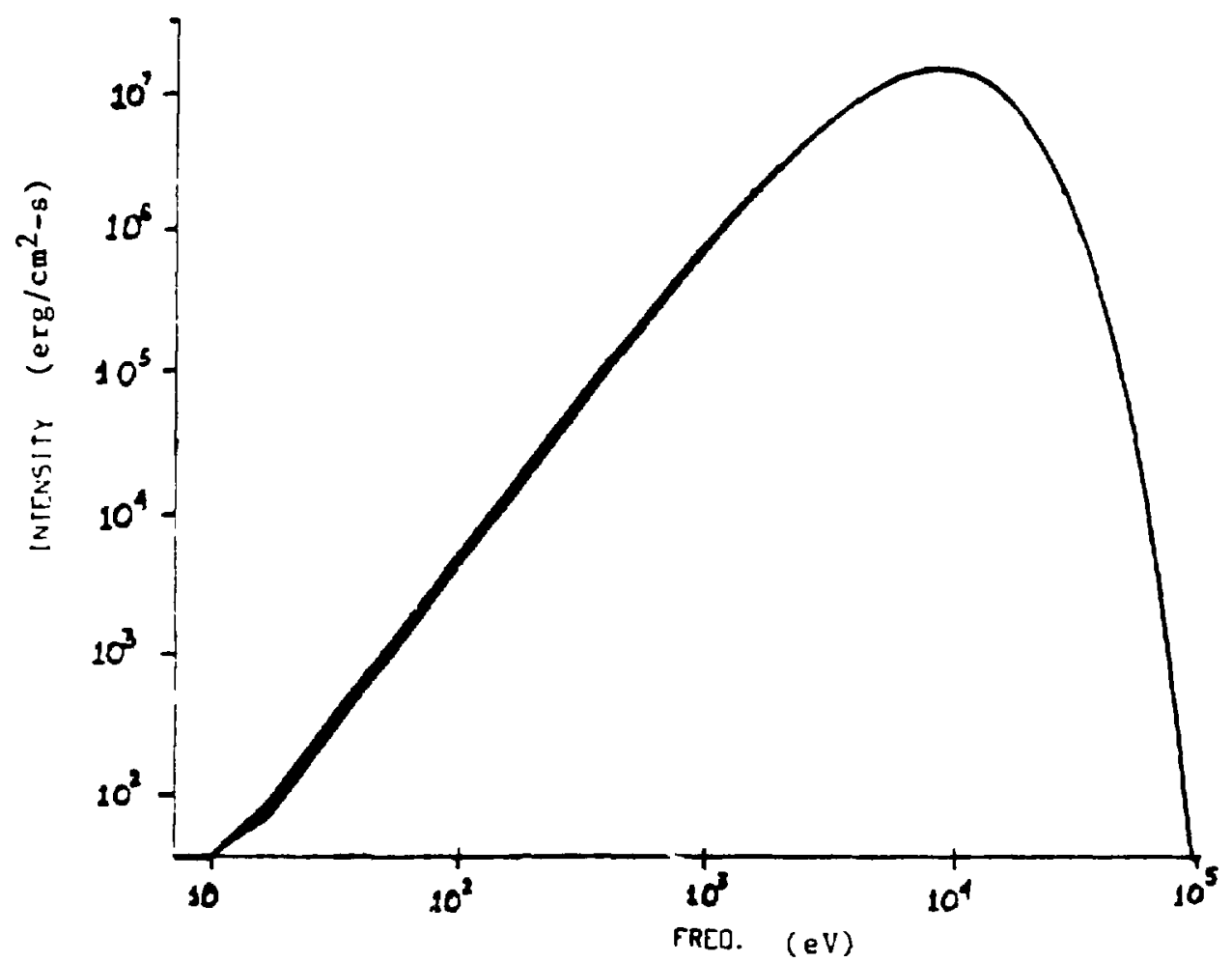

Figure 5.

Time snapshots of the 1sotropic 1ntensity versus frequenc:

for elapsed t1me $4 \times 10^{-5}$ seconds. 


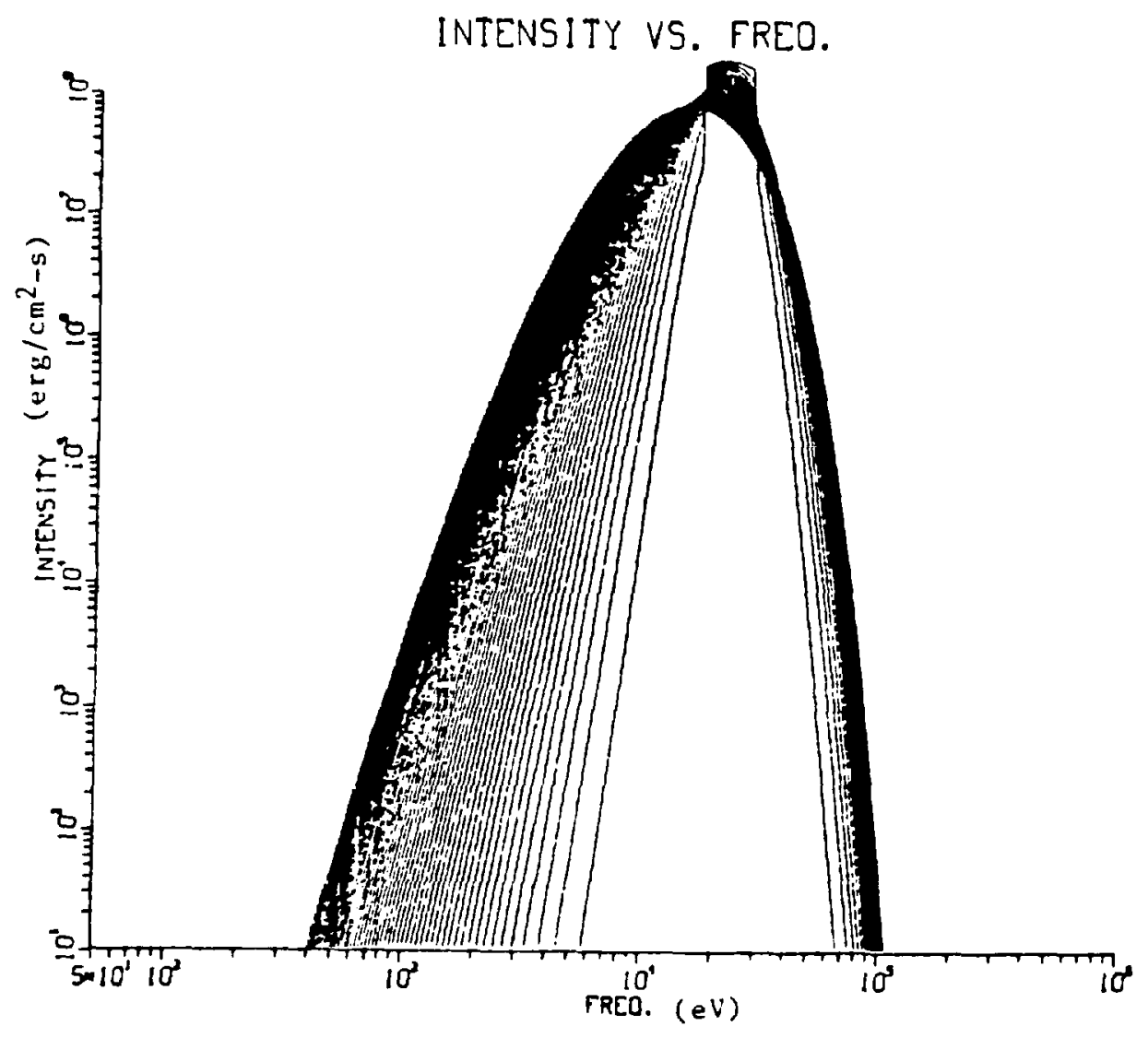

Figure 6.

Time snapshots of the isotropic intensicy versus frequency with t1me step $4 \times 10^{-7}$ seconds and elapsed time $2 \times 10^{-5}$ seconds. 
Another initial value problem studied is similar to that in Fig. (6). In this problem, an additional spectral feature is added. A rectangular feature of lower magnitude at a lower frequency is added. This problem is illustrated in several figures for clarity. Figure (7) shows the initial distribution and the first few time steps. Figure (8) shows the spectral profile for several cime steps near the completion of the run. Finally, Fig. (9) shows the protile with the superimposed curves at all times. A total of luo time stens was used. Each time step was $-5 \times 10^{-7}$ seconds in duration. Again, tise initial distribution relaxes coward equilibriut.

The number of photons was conserved in all of the classes of problems run. One example of this is for the inicial valde problem... illustrated in Fig. (7). Over the duration of the run, the difference between the initial number of photons and the final number of photons was found to be $.09 \%$

Since the evolution of the spectral profile in problems in which Induced processes are highly important is dependent upon the initial profile, direct comparisons were difficult. Hovever, several definitive comparisons were found. One example of an induced Compton effect was found by Monces(14) and Zel'dovich and Syunyaev. (25)

Montes foind that, for an initial broad Gaussian spectrum in the region where induced effects dominate, the lower end of the Gaussian begins to break into features which appear to be a series of photon solitons moving downward in frequency space. In this case, the higher amplitude solitons appear at the lower frequencies. Zel'dovich and Syunyaev found this same type of behavior in a problem 


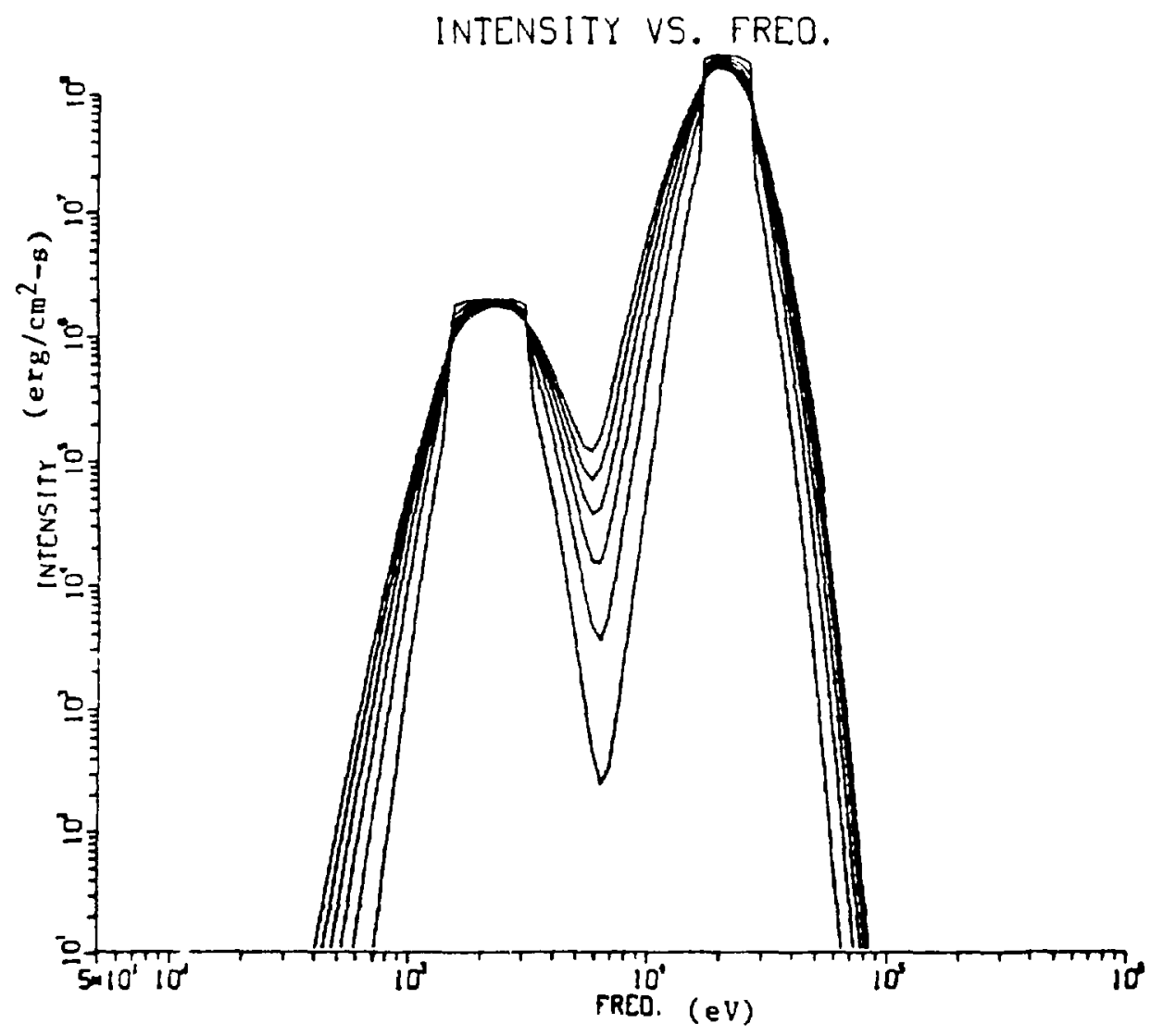

Figure 7 .

Time snapshots of the isotropic intensity versus frequency for beginning of computer run with a time step of $4 \times 10^{-7}$ seconds. 


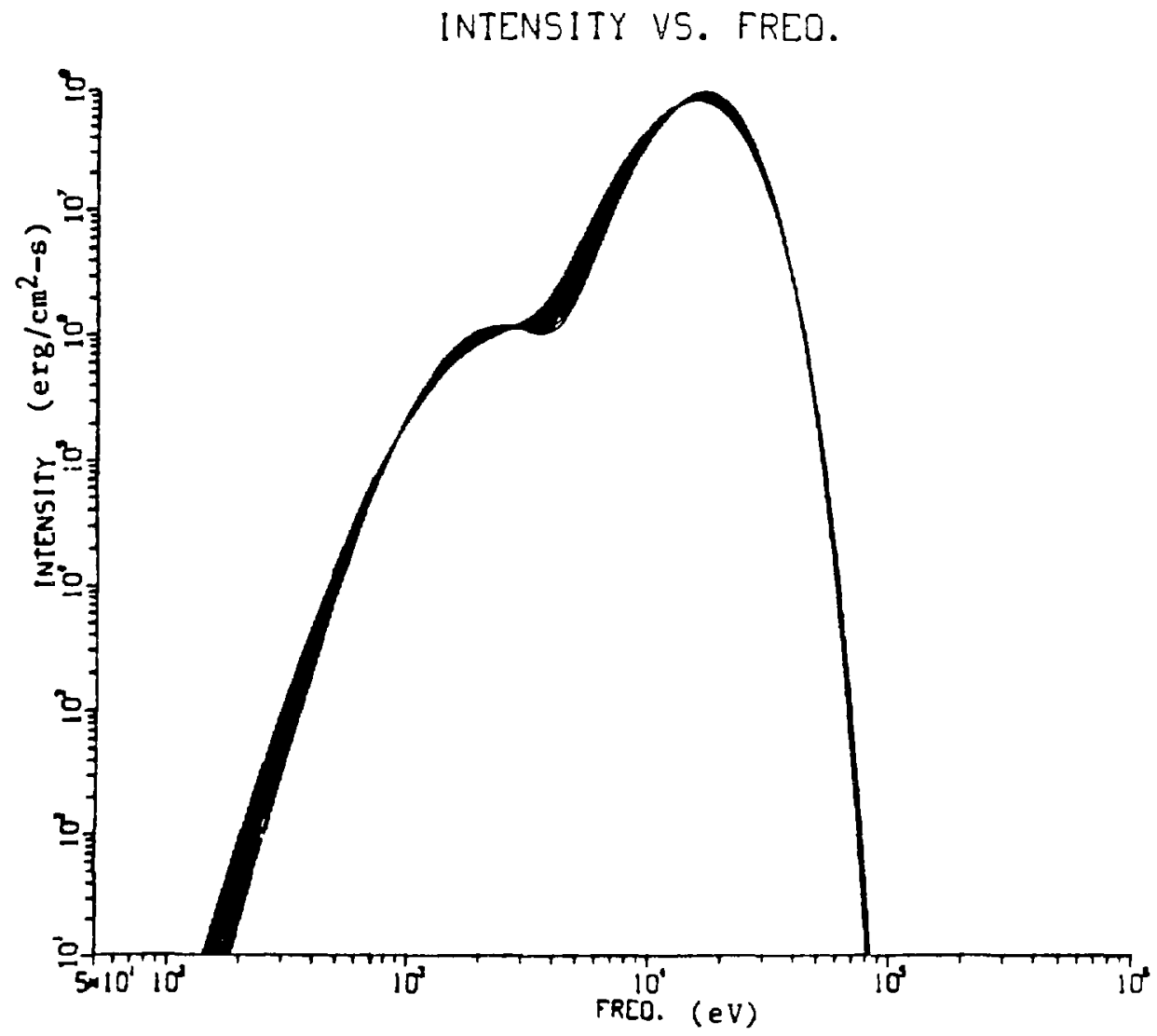

Figure 8 .

Time snapshots of the isotropic intensity versus frequency

near the completion of the computer run with time step $4 \times 10^{-7}$ seconds. 


$$
M
$$


In which the Initial spectrum was that of a step function. An example of this type of problem was run using the code developed here. It was set up to approximate the results quoted by Montes. In this case the initial spectrum is that of a very broad Gaussian. The Gaussian peak is less than an order of magnitude different from that given by Montes. The width of the spectrum is significantly larger. The same behavior noted by Montes was found to occur. The peak of the Gaussian is centered at $4 \mathrm{keV}$. Two problems were run, one in which the electron temperature was at $100 \mathrm{eV}$, and one with the electron temperature at lokev. In both cases, the soliton-like features found by Montes appeared when induced scattering was Included. These features did not occur when induced scattering was neglected. In the case of $100 \mathrm{~V}$ electrons, the solitons appear and begin to move downward in frequency space.(Figure 10) In the case of lokev electrons, a very interesting phenomenon occurs. The features appear and grow in amplitude. However, these soliton-like features do not move downward but remain in position. This is illustrated in Fig. (11). Figures (10) and (11) show the lower frequency end of the Gaussian tail where the solitons appear. Fluctuations which occur in the lower two frequency groups have been ignored as described on Page 92.

One type of behavior noted in the literature applied to spectral features moving downward in frequency space.(11,12,13) Induced Compton scattering results in the steepening of the red side or lower frequency end of the feature. This behavior also occurred using the techniques considered in this thesis. Figure (12) shows an initial 


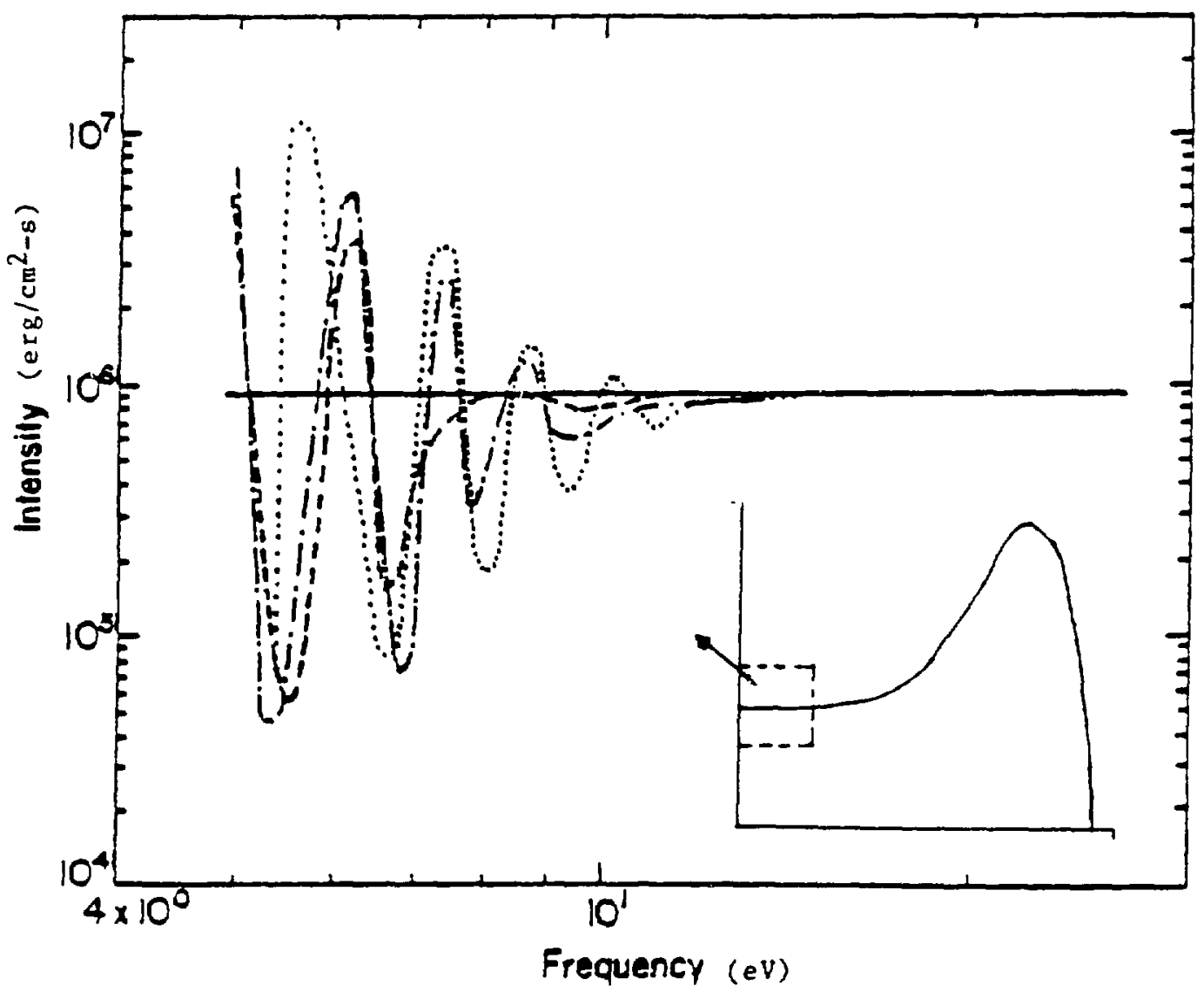

Figure 10 .

Plot of the Isotropic Intensicy versus frequency for the Inftal Gaussian profile shown in the lower right hand corner with time step $3 \times 10^{-8}$ seconds. 


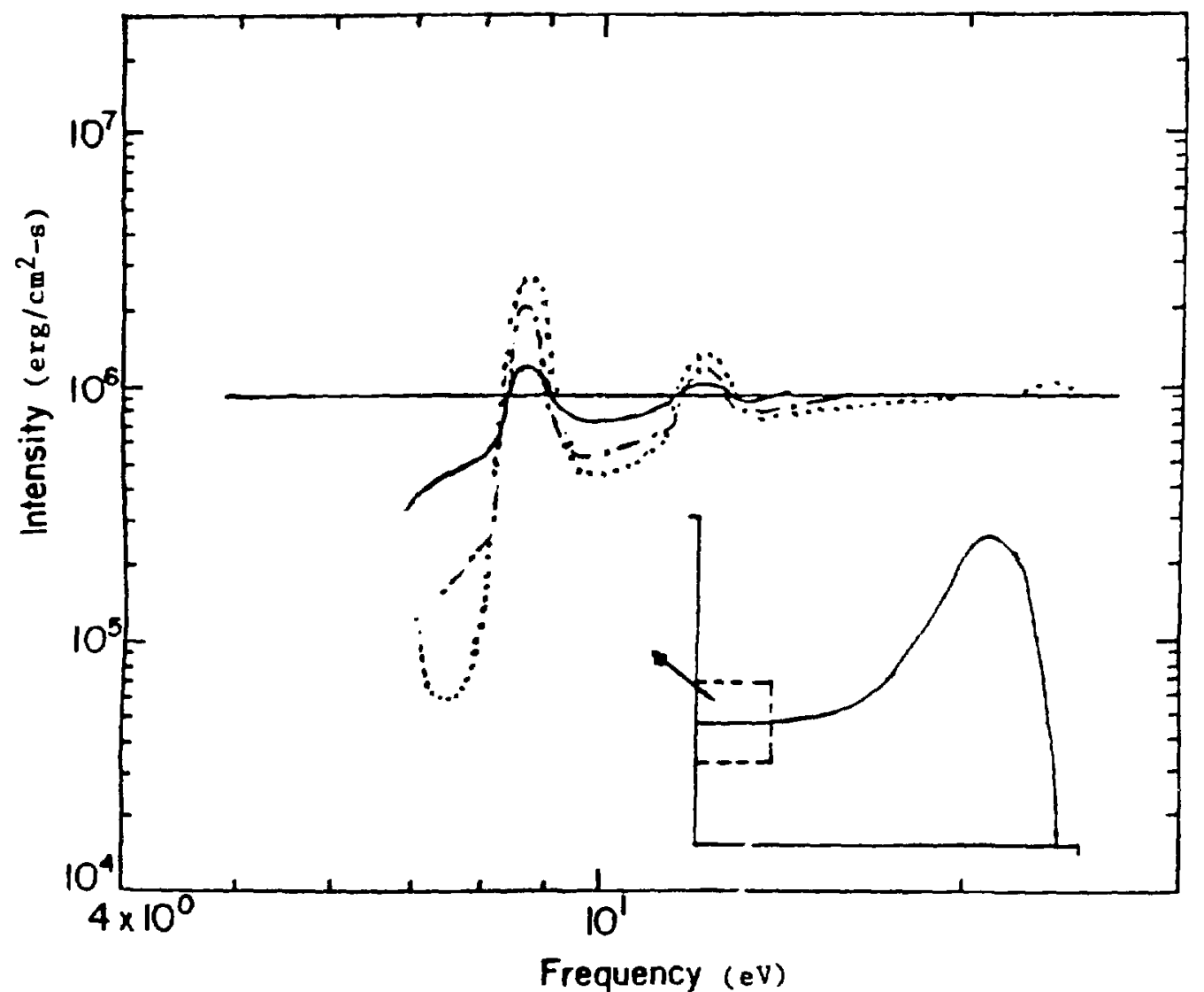

Figure 11 .

Plot of the isotropic intensity versus frequency for the Initial Gaussian profile shown in the lower right hand corner with time step $3 \times 10^{-8}$ seconds. 


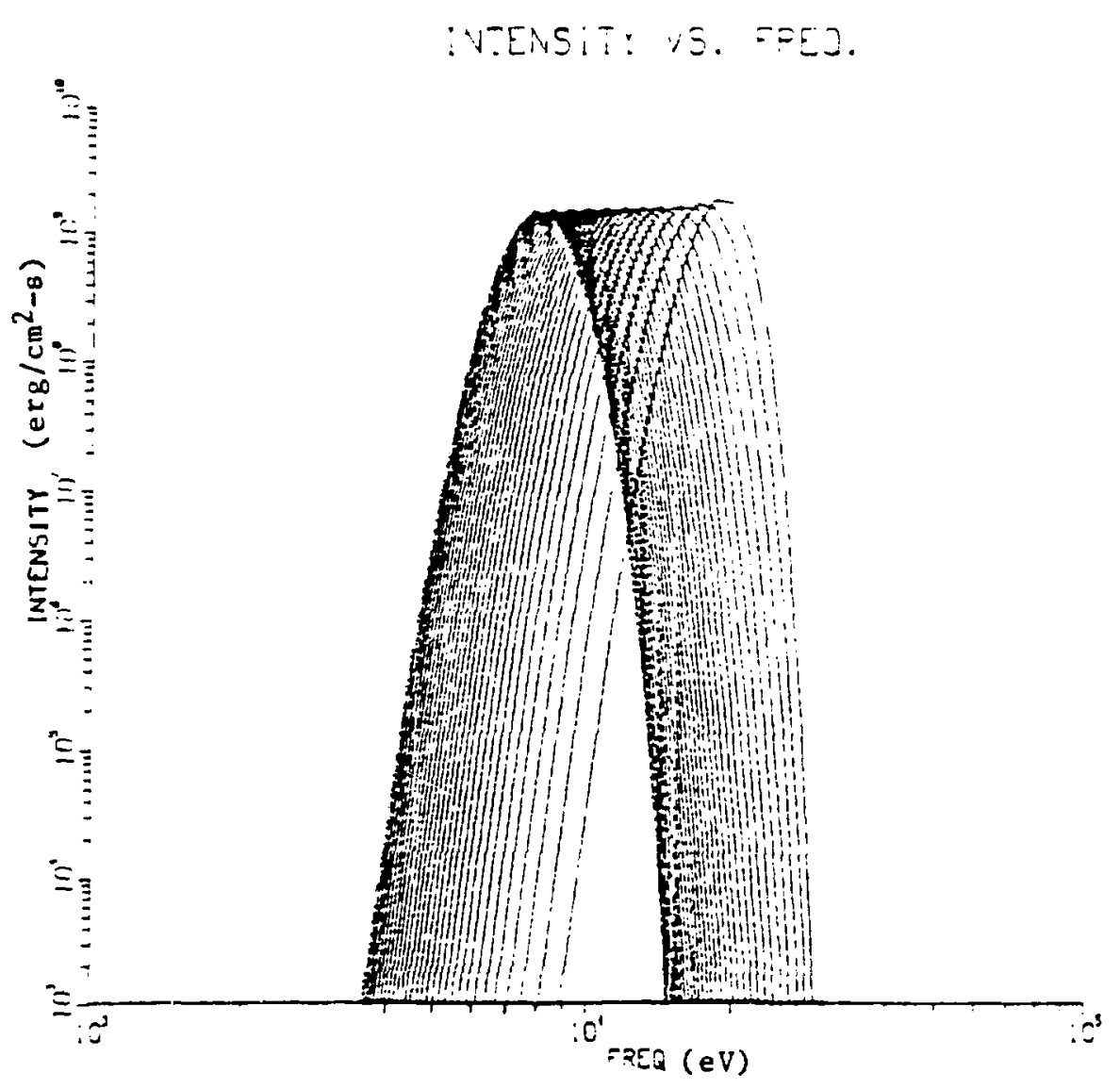

Figure 12.

Iime snapshots of the isotropic intensity versus frequency with time step $2 \times 10^{-7}$ seconds and elapsed time $6 \times 10^{-6}$ seconds. 
Gaussian profile moving downward in frequency space. The profile is centered at $20 \mathrm{keV}$ and is scatering in an electron field at $100 \mathrm{eV}$. Figure (12) does not include induced scattering. Figure (13) shows the same problem with induced scattering included. Figure (13) clearly shows a red side steepening as the photons begin to pile up at the low frequency end of the spectral feature.

Another interesting behavior has been noted by Montes. (14) In this case, an initially narrow Gaussian spectrum scattering downward in frequency space decreases in speed, grows in amplitude and narrows in width. The behavior looks like a photon soliton. This behavior is not unique, however, to Induced scattering problems. Similar behavior was found in a problem where linear scattering terms dominate. Figure (14) shows a problem which exhibits this behavior. The initial distribution used is rectangular. However, it rapidy becomes roughly Gaussian. The same general behavior noted by Mantes was found to occur. However, after a period of time, the profile begins to decrease in amplitude and slowly spread out in frequency space.

An extremely interesting problem which was addressed for this thesis involved an initlal photon spectrum in which two distinct features are present initially. The features are both represented by step functions in frequency space and are of the same amplicude. This is essentially the same problem as that given in Fig. (7) with 
IVEVSITY VS. FAEG.

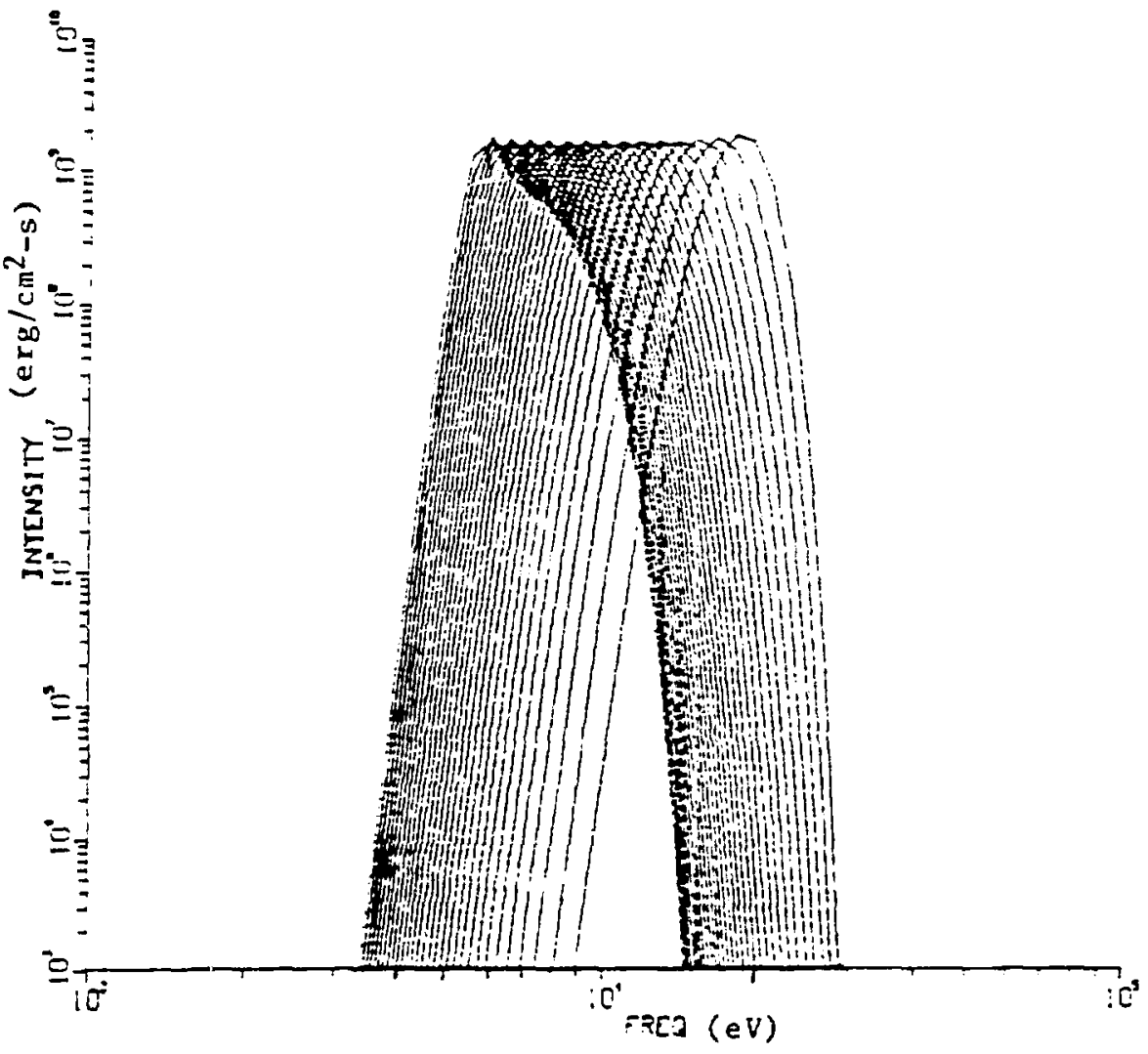

Figure 13.

iime snapshots of the isotropic intensity versus frequency with time step $2 \times 10^{-7}$ seconds and elapsed time $6 \times 10^{-6}$ seconds. 
the lower frequency spectral feature increased in amplitude. This increase in magnitude of the lower frequency feature causes the quadratic terms in the transport equation to be large enough $t$. contribute significantly to the solution. This causes an enhancenent of the downscattering from the upper peak as well as the lower frequency peak. Hence, the radiation sweeps downiard in frecuen: space. The enhanced downscatcering fills the lower frequencies with enough radiation to maintain the importance of the inductet tiect. Hence, the radiation profile "bootstraps" itself dowinard in frequency space. Figure (15) illustrates this problem when inducet effects are not included. Figure (16) demonstrates the same frubles. when induced scatcering is included. The induced efiect is quite visible.

Another problem with is of great interest is the rule of induced scattering on the time it takes for an equilibriun, i.e., blackbody distribution to equilibrate wjth an electron field heid at a different temperature. Two problems of this type are addressed. In the first, a blackbody radiation field at $4 k e v$ is placed in an electron field fixed at lokev. The other case has the radiation at lokeV and the electrons flxed at $4 \mathrm{keV}$. It was found that in the case of hot radiation in a cooler electron field, induced scattering enhanced the cooling of the radiation field to a small degree. In this case, the radiation energy density af ter . $2 \times 10^{-4}$ 


\section{INTENSITY VS. FREO.}

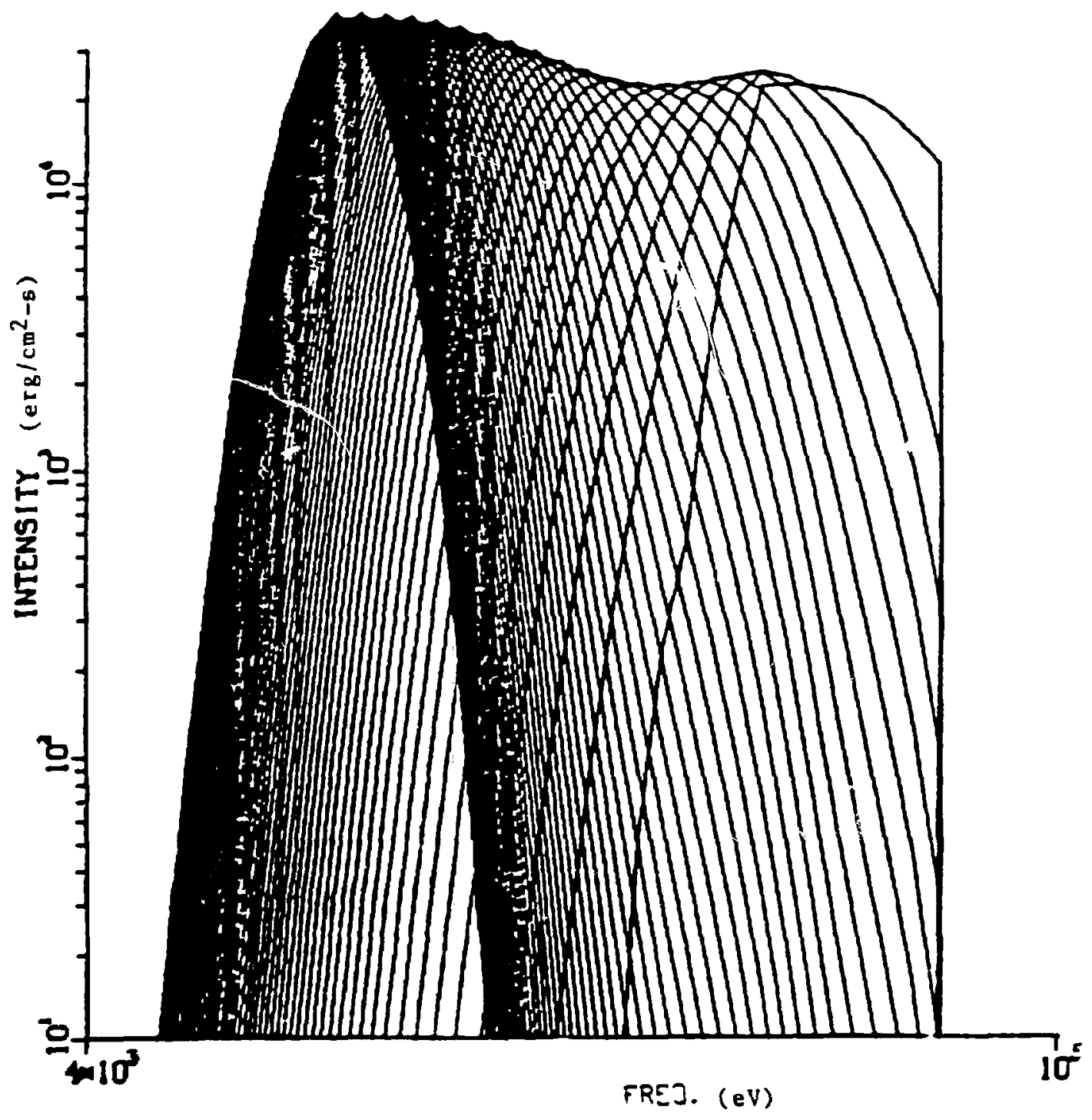

Figure 14.

Time snapshots of the 1sotropic intensity versus frequency with time step $4 \times 10^{-7}$ seconds and elapsed time $4 \times 10^{-5}$ seconds. 


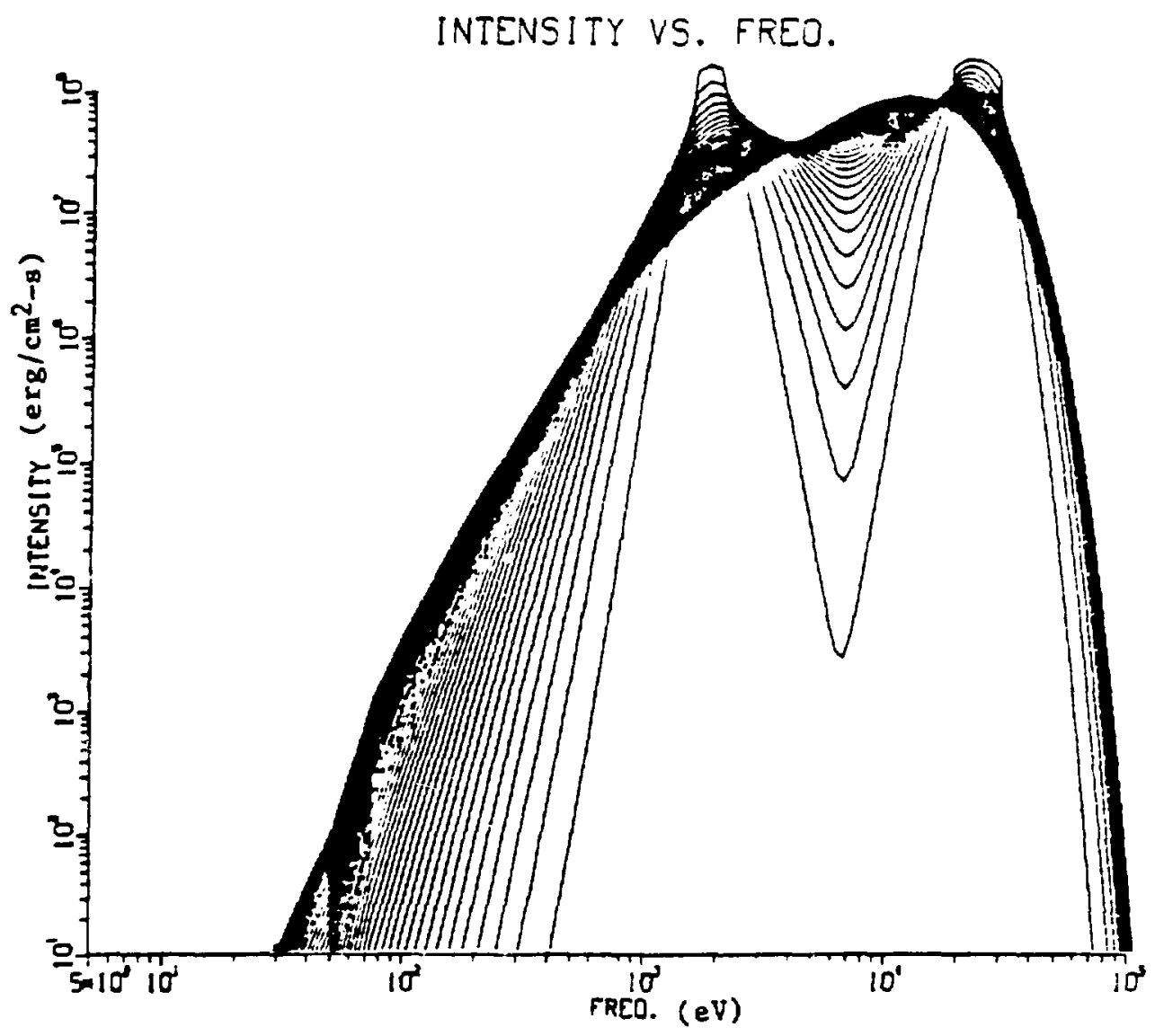

Figure 15.

Time snapshots of the isotropic intensity versus frequency with time step $4 \times 10^{-7}$ seconds and elapsed time $2 \times 10^{-5}$ seconds. 


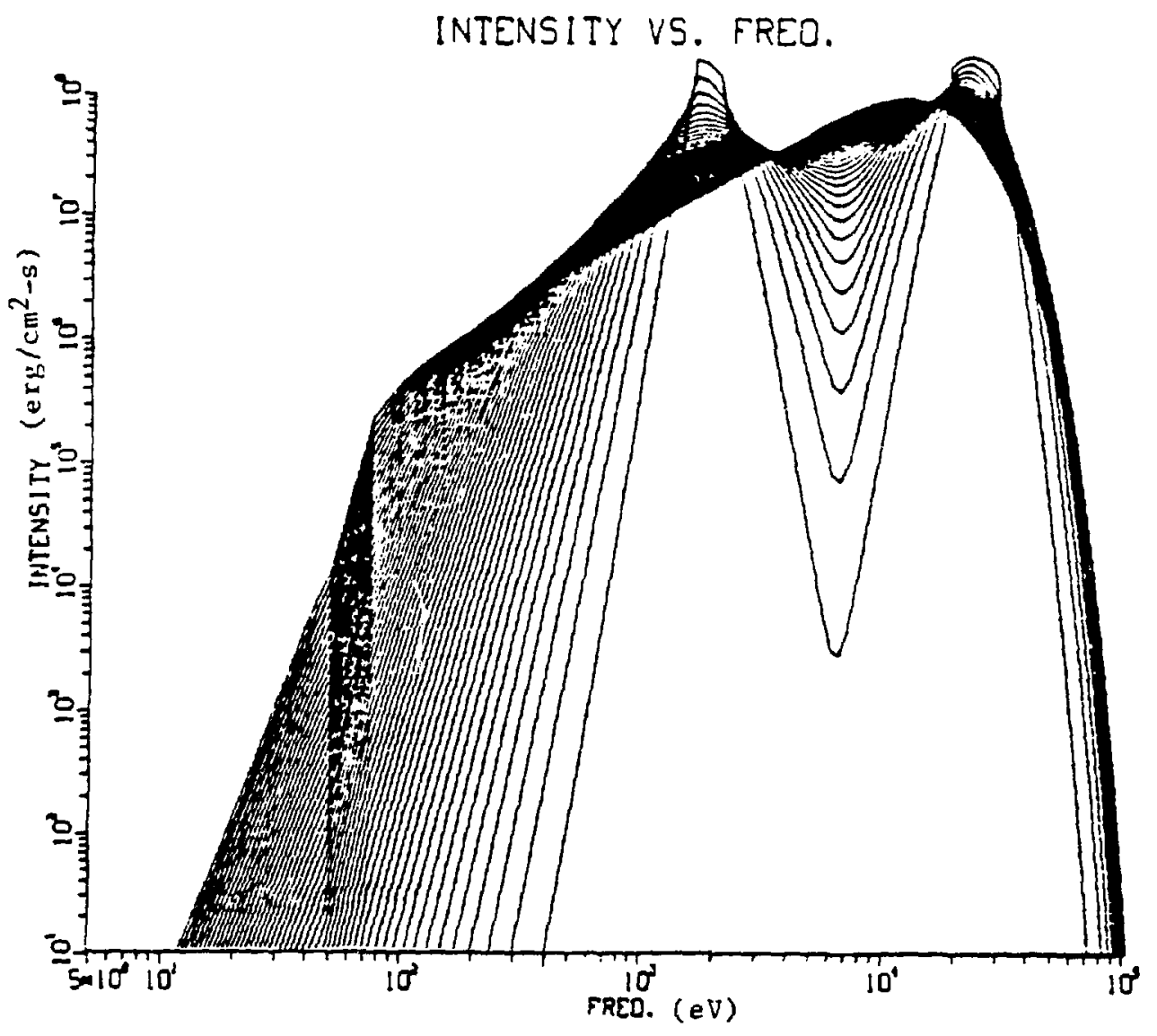

Eigure 16.

Time snapshots of the isotropic intensity versus frequency with time step $4 \times 10^{-7}$ seconds and elapsed $t$ ime $2 \times 10^{-5}$ seconds. 
seconds was $2 \%$ lower when induced scattering was included than when it was not.

The opposite occurred for the case of hot electrons and cooler radiation only to a much smaller degree. In this case, the induced scattering slowed the heating of the radiation. After $.2 \times 10^{-4}$ seconds, the difference was only $.1 \%$. This is consistent with other results in which induced Compton scattering was found to enhance the heating of electrons. (13)

Some interesting information is obtained if we allow the electron temperature to vary after each time step. Several problems of this type were solved. This was accomplished by simply requiring conservation of energy. If the radiation energy density at time $t_{\text {, }}$ is given by $E\left(t_{0}\right)$, and if the electron energy density is given by $u\left(t_{0}\right)$, then, at a later time $t$, the new electron energy density is found from the relation

$$
u(t)=u\left(t_{0}\right)+E\left(t_{0}\right)-E(t) \text {. }
$$

The new electron temperature is calculated fron Eq. (223). The graphics output for these problems shows the changes in electron temperature versus time when Induced scattering is both included and excluded.

In the problem of an initial blackbody radiation field in an electron field of dif'erent temperature, little effect due to induced scattering was observed. In one case, the electrons are initially at $100 \mathrm{eV}$ while the radiation is at lokev. A slight separation can be 
seen in the temperature curves shown in Fig. (17). The upper curve is that of the electron temperature when induced effects are included. The lower does not include these effects. This is consistent with what was observed when the electron temperature bas held constant. Figure (18) shows the problem where the electrons art at $20 \mathrm{keV}$ and the radiation is at $4 \mathrm{keV}$. No separation was observable in the graphics, and only a slight separation could be observed in the numerical output.

The more interestang cases occurred when the initial spectrum was similar to that shown in Figs. (15) and (16). In all of these cases, the upper curves represent inclusion of induced scatcering, while the lower curves exclude the induced effect. Figure (19) show's a problem where the initial photon distribution contains two spectral features as in Fig. (15). In this example, both features are of the same magnitude. The electron temperature is higher where induced scattering is included due to the sweefing down of the radiation profile in frequency space caused by induced scattering.

If the lower spectral feature in the above problem is increased by an order of magnitude, the effect of induced scatcering on the electron temperature becomes even more dramatic. This is illustrated by Figs. (20) and (21). Figures (20) and (21) show the same problem solved on different time scales. It can be seen that in these examples, the electron temperature actually decreased without induced scattering and increased when induced effects were included. 


\section{ELEC TEMP VS. TIME}

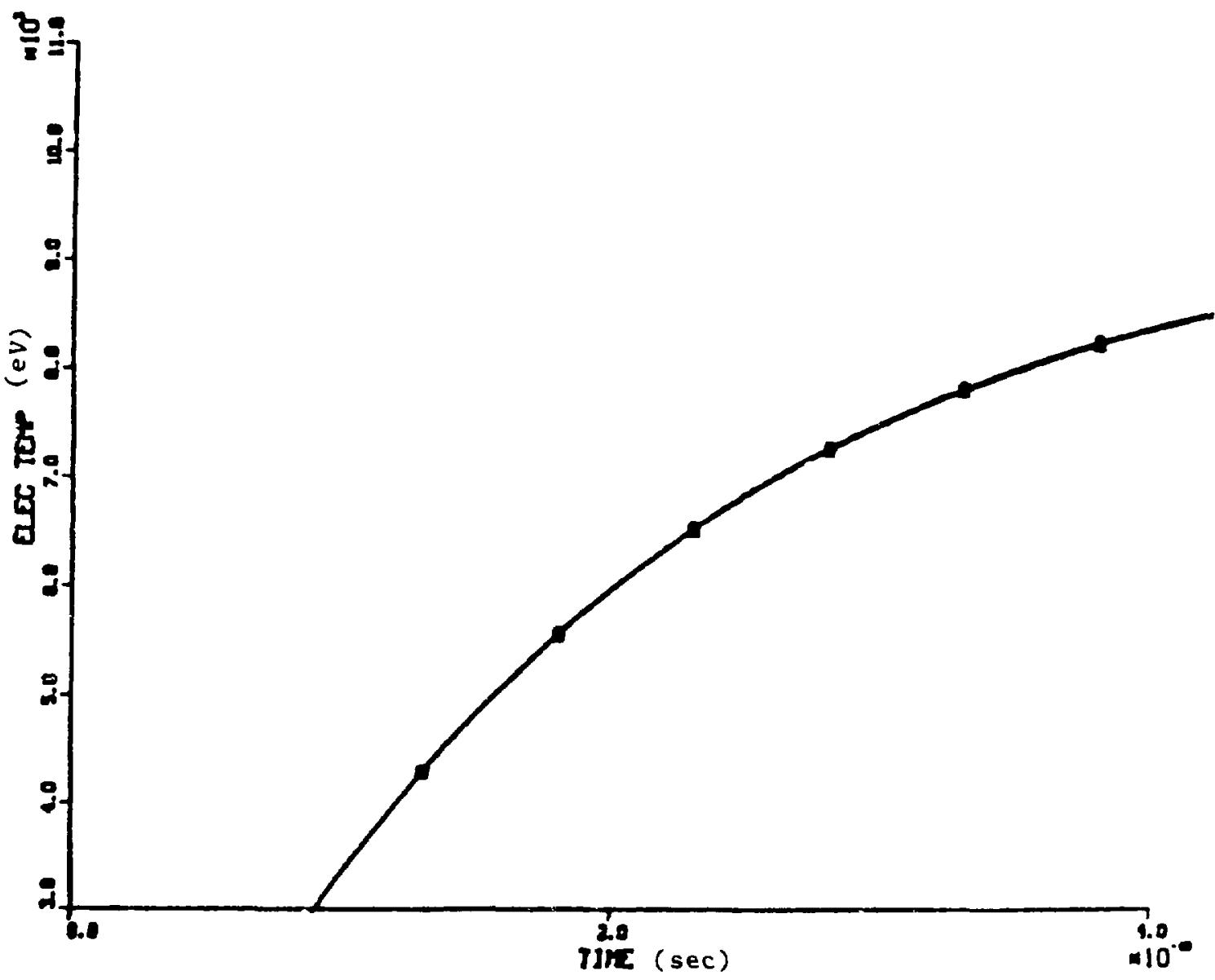

Elgure 17.

Plot of electron temperature versus time for both induced scattering and linear scattering. 


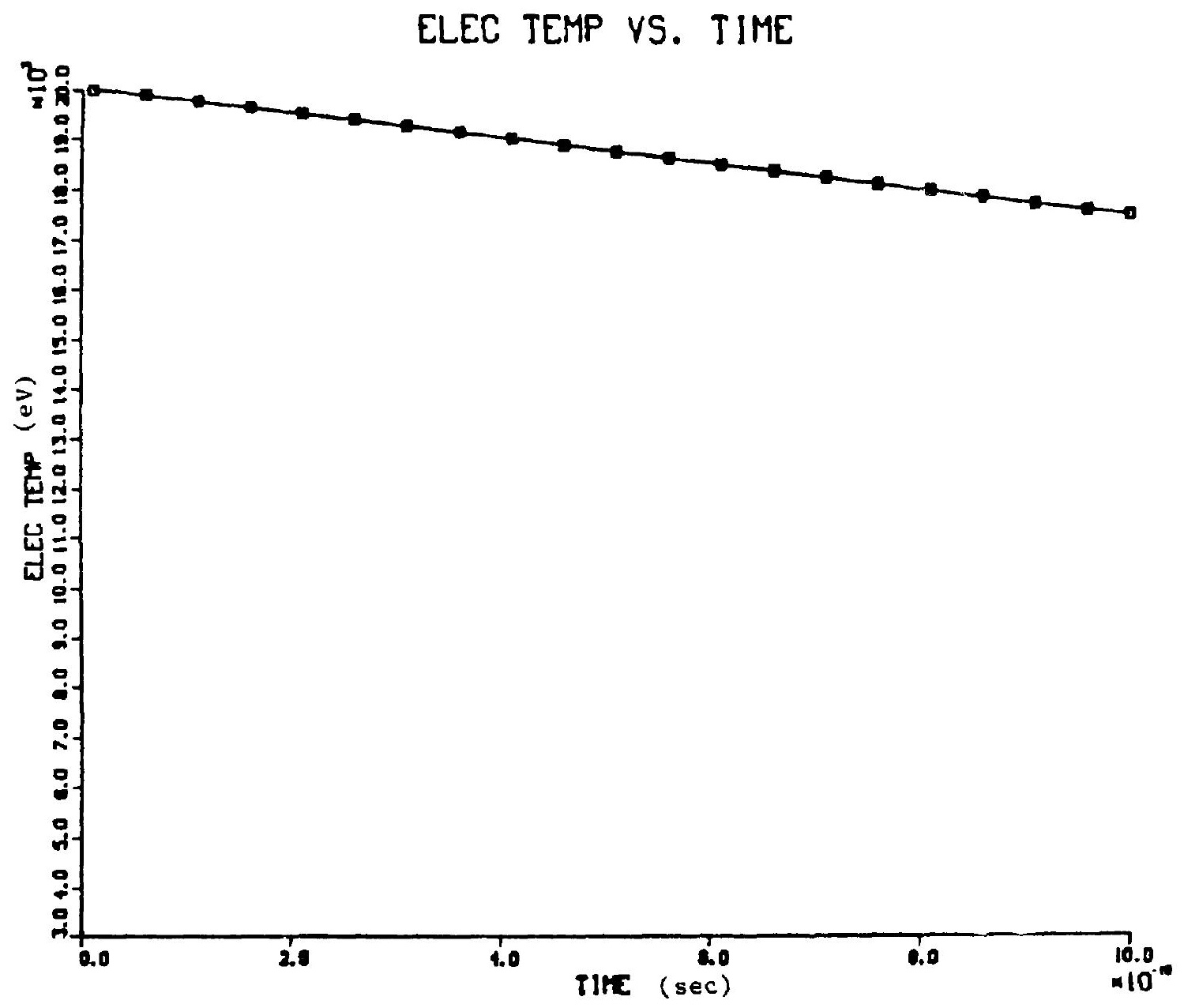

Figure 18.

Plot of electron temperature versus t1me for both induced scattering and linear scattering. 


\section{ELES TEMF V. TIME}

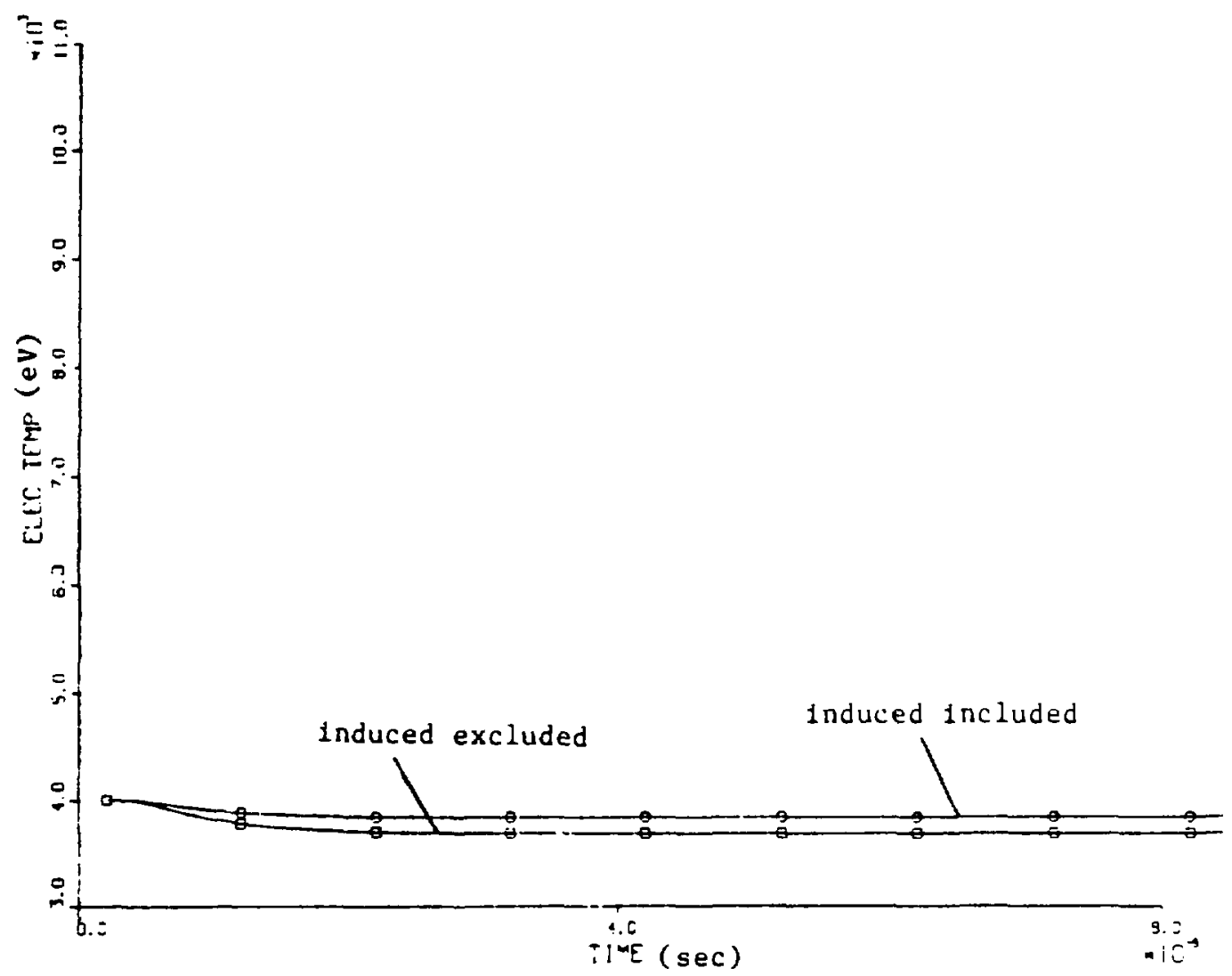

Figure 19.

Plot of electron temperature versus time for both induced

scattering and linear scattering. 


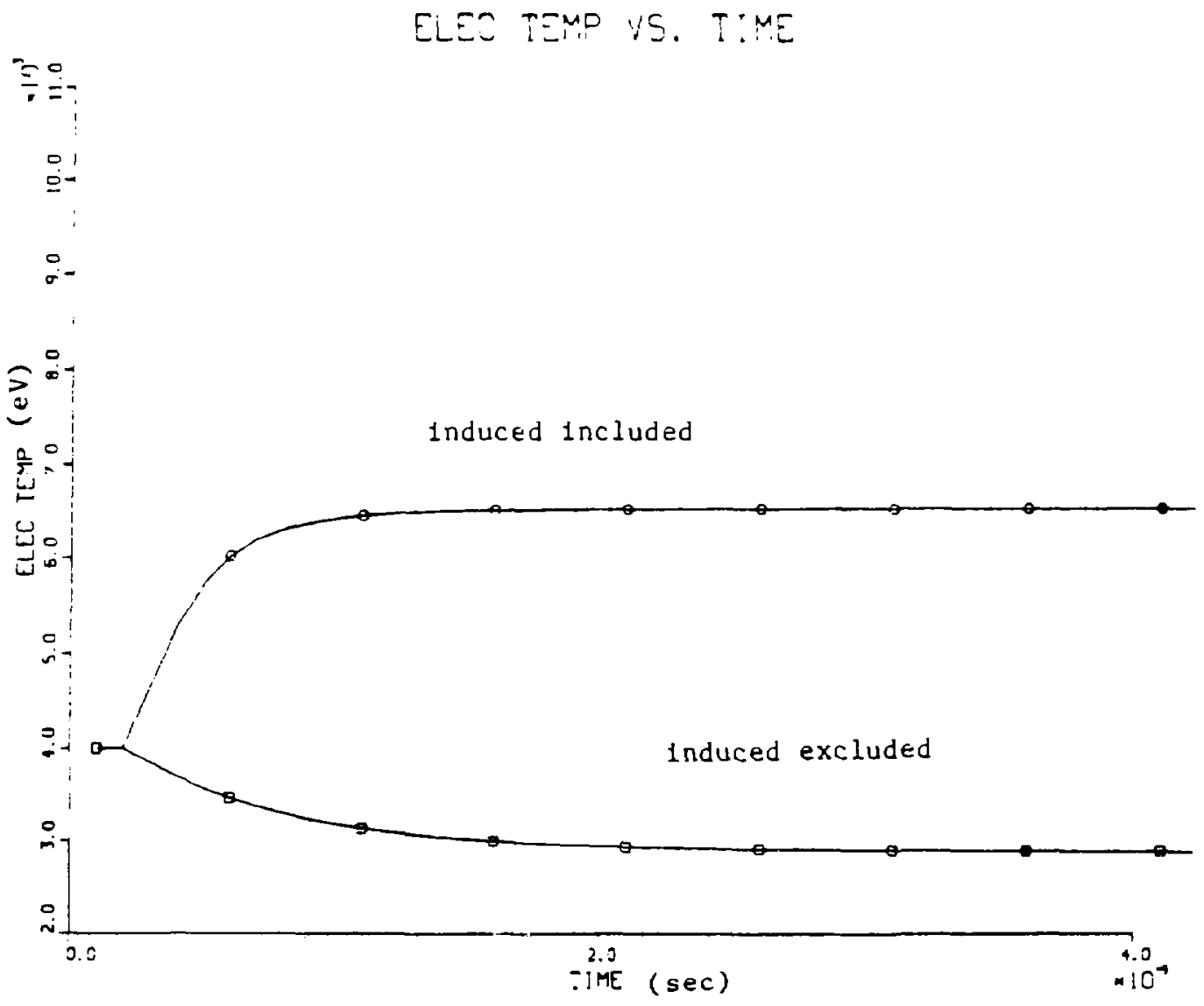

Figure 20.

Plot of electron temperature versus time for both induced scattering and linear scattering. 


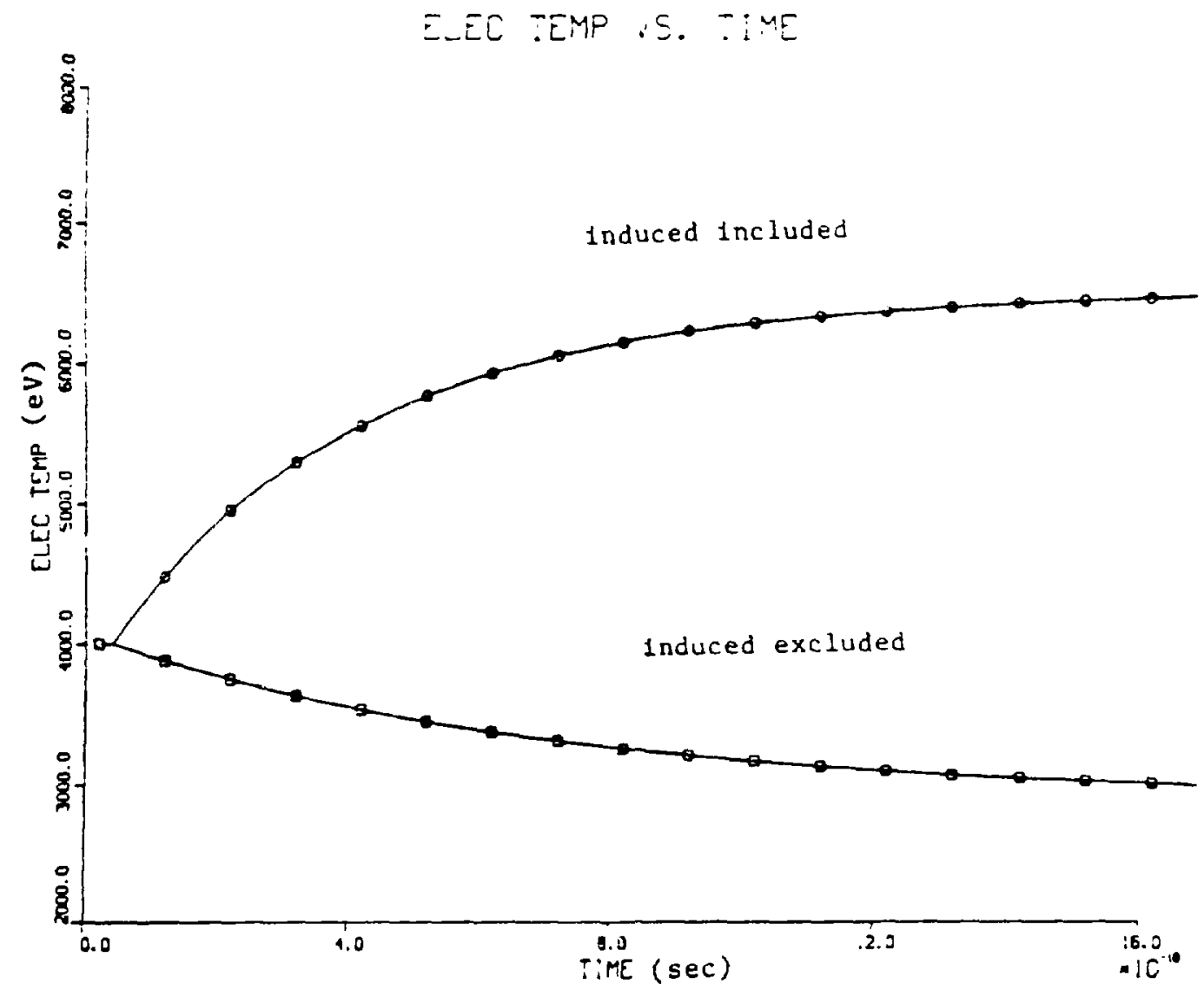

Figure 21.

Plot of electron temperature versus time for both induced scattering and linear scattering. 
4.2 Spatially Dependent Solutions

The problems considered above have all excluded spatial dependence. The codes developed were designed to handle this dependence. Several examples of the ability of the codes to do this are given here. One is run to demonstrate the ability of the method to handle discontinuous problems. In this case, the scattering effects were nealected, and the isotropic intensity is plotted as a function of radius. Hence, only the streaming terms wert included in the transport equation. These equations are

$$
\frac{\partial v_{0}}{\partial t}+\lambda_{1} \frac{\partial v_{0}}{\partial r}+\frac{c}{\sqrt{3} r}\left(v_{0}-v_{1}\right)=0
$$

and

$$
\frac{\partial v_{1}}{\partial t}+\lambda_{2} \frac{\partial v_{1}}{\partial r}+\frac{c}{\sqrt{3} r}\left(v_{0}-v_{1}\right)=0 \text {. }
$$

In the second example, scatcering and induced scattering effects are included, and a well-defined radiation wave is shown.

The ability of the method of characteristics to handle a wide variety of spatially dependent initial and boundary conditions is illustrated by Fig. (22). In this example, a spatially dependent problem is solved in an infinite domain without scattering effects. The Inftial photon distribution has a discontinuity in it at a finite radius $r_{0}$ In the region $0<r<r_{0}$, all the radiation 1 s moving 
radially outward. For the region where $r>r_{0}$, the radiation field is 1sotropic. Figure (22) is a plot of the isotropic portion of the radiation, 1.e., $I_{0}(\nu)$. The isotropic flux fills in the area $0<r<r_{0}$, while the outward current initially in the same region causes an outwardly traveling wave. The radiation filling the center region converges on the center and reflects. This creates a second outgoing wave. The method of characteristics handles these traveling waves and simple reflective boundary conditions quite nicely at $r=C$.

Figure (23) shows an example of the use of the code to solve problems when scattering and spatial effects are included. In this case, an isotropic radiation field is introduced in a finite region centered at the origin. This radiation is then allowed to travel outward while scattering of of the electrons. What results is a we1.1-defined radiation wave traveling outward. This problem required approximately twenty-five minutes of CIAY computer time to run. Problems where spatial dependence is suppressed required less than three minutes of conputer time. 


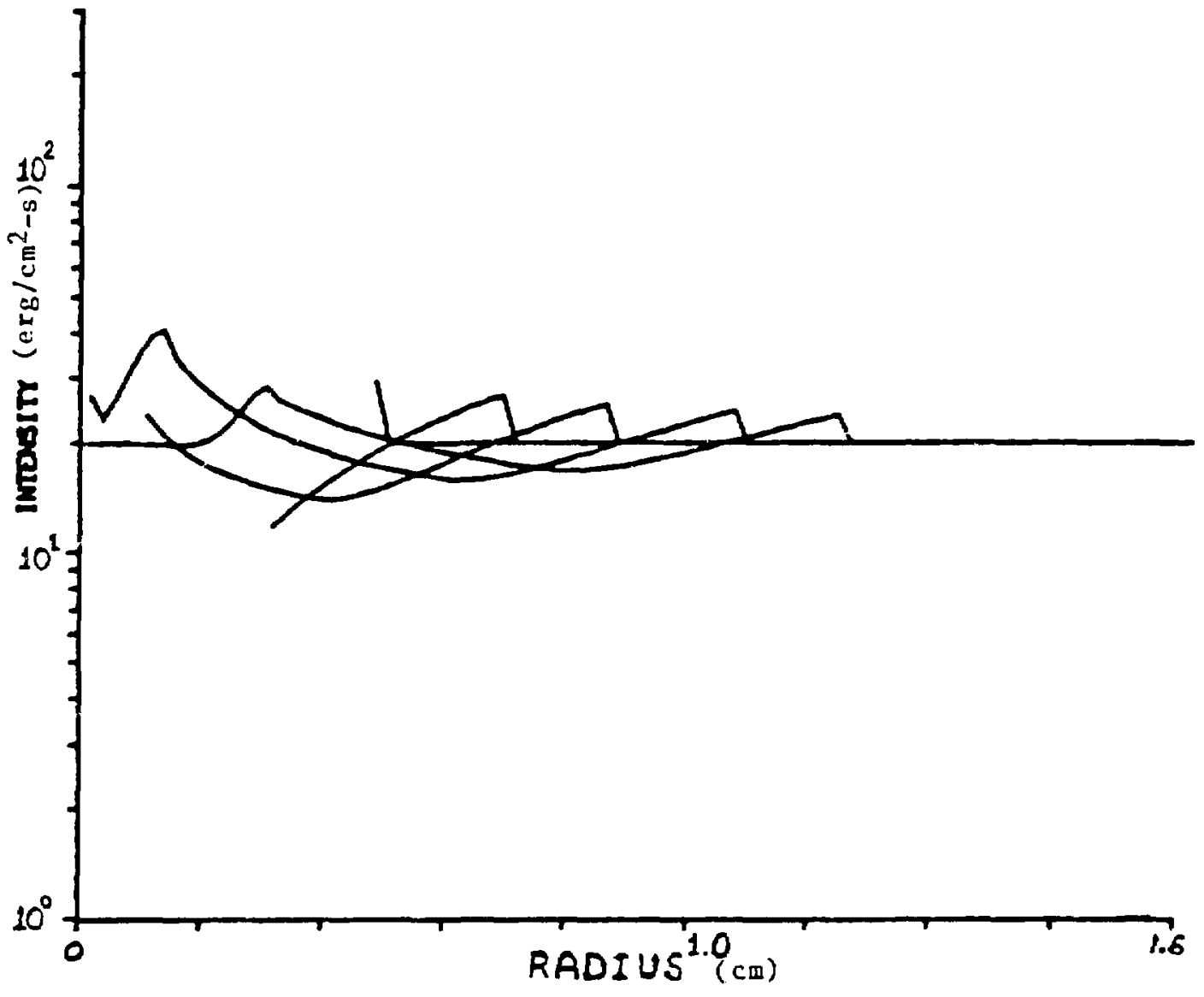

Figure 22.

Time snapshots of the isotropic intensity versus radius with time step $4.6 \times 10^{-11}$ scconds. 


\section{ENERGY GENSITY}

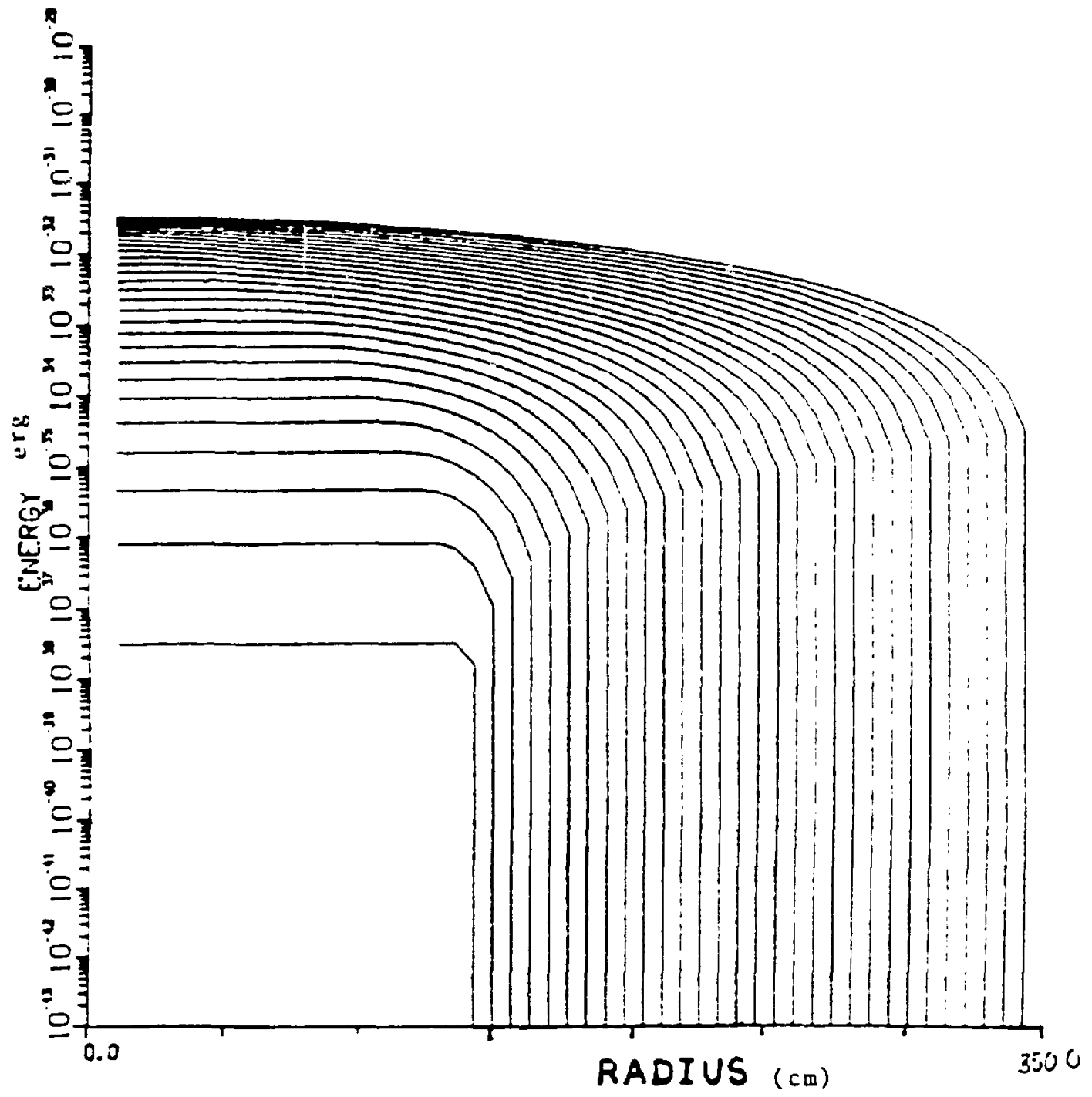

Figure 23.

Time snapshots of the radiation energy density versus radius with time step $4 \times 10^{-10}$ seconds. 
5. CONCLUSIONS AND RECOMENUATIONS FOR FURTHER STUDY

\subsection{Conclusions}

The problems studied in this thesis are those in which both induced Compton scattering and linear Compton scattering are important. Problems of this type were not found in the published literature. The approach taken was as follows.

The integro-differential radiation transport equation with the inclusion of induced effects was the starting point. The $P_{N}$ equations were derived with the inclusion of induced effects. The problems solved utilize the $P_{1}$ equations. Several assumptions are now made. Absorption and emission are neglected. This is because an electron gas was used as the transport medium. Compton and inverse Compton are the scattering processes which were examined. The Pl equations were reduced to canonical form and solved using the method of characteristics.

The techniques applied in this thesis verified several results found in the literature.(13,14) The effect of the initial photon spectrum on resulting time evolution of the spectrum was verified. It was also verifled that a sharp spectral feature moving downward in frequency space changed as a result of 1nduced scattering. Induced scattering caused a steepening of the low frequency edge. 
It was found that induced scattering produced its most interesting effects when distinctive spectral features represented by step functions in frequency space were present. Because of the quadratic nonlinearity in the transport equation, the effect scales as the square of the intensity and inversely as the cube of the frequency. Hence, spectral features of high incensity at lower frequencies showed the induced effects most dramatically. These features, as they scattered down in frequency space, tended to pull the radiation spectrum downward in frequency space due to the induced scattering effect. As the radiation scatters downward in energy, the effect in many cases would increase due to the inverse dependence on the cube of the frequency. This increase in magnitude of the effect was greater than the decrease caused by the decline in the magnitude of the intensity. This process is best described as a sweeping down of the intensity spectral profile when induced scattering was present.

The effect of induced scattering on the electron temperature was also of interest. It was found that an equilibrium radiation field scattering in an electron field of different temperature showed a small but observable effect due to induced scattering. Induced scattering tended to enhance the cooling of the radiation field when the radiation temperature was initially hotter than the electron field, but the oppasite accurred when the electrons were hot ter than the radiation field. 
In problems where the sweeping down in frequency space of the radiation occurred, the electrons were always hot ter when induced scatering was present than when the effect was not included. In some cases, the electrons would actually increase in temperature when induced scattering was included, and decrease in temperature when it was not.

The capacity to solve spatially dependent problems with induced effects included was also not avallable from prior work. The techniques and codes developed in this thesis have this capacity. This was demonstrated in Chapter Four.

Although induced scattering effects are extremely dependent upon the initial photon spectrum, several rules of thumb can be stated. These apply when both linear and nonlinear effects are important . When addressing a radiation transport problem in this regime, several conclusions can be drawn.

If the problem involves equilibrium radiaion, i.e., a blackbody spectrum without a significant difference in temperature from the electron field, induced Compton scattering can be ignored without significantly affecting the results. If this temperature difference is significant, inclusion of induced Compton scattering will yield more accurate results. However, good "ballpark" results can be obtained if induced scattering is neglected.

If, however, problems which include sharp spectral features are of Interest, neglecting Induced Compton scattering can result in answers which are significantly in error. In cases where the spectral features are of a high order of magnitude at low 
frequencies, inclusion of induced Compton scattering becomes Imperaitive. A quick check of the importance of induced scattering can be done by using the criterion

$\mathrm{N}>1$

where

$$
N=\frac{c^{2} J(v)}{2 h v^{3}} .
$$

In problems where $\mathrm{N} \ll<1$, induced Compton scattering can be neglected.

\subsection{Recommendations for Further Study}

Several avenues of further investigation could be pursued to provide further knowledge of induced Compton scattering in radiation transport. One of these is the study of induced scattering effects on the directional dependence of the radiation. One example of this type of problem is related to inertial confinement fusion. Consider a fusion target which is rapidly burning in the center of the pellet. The directional dependence of the radiation should be highly peaked in the radial direction. How does induced Compton scattering effect the radial cransport of the radiation? 
This sort of problem could be addressed by solving a higher order $P_{A}$ approximation, such as a $P_{3}$ approximation.

Another problem of interest would be to include a kinetic description of the electron field. Simultaneous solution for the electron velocity distribution and the radiation energy distribution would be quite interesting. Solution of this problem would establish quantitative criteria relevant to the validity of the assumption in this thesis that the electron distribution remains Maxwellian. 


\section{ACKNOWLEDGMENIS}

I would like to acknowledge the guidence and advice given to me by my advisor, Professor Roy A. Axford, on the work performed i. this thesis. His help and patience were invaluable. Prof. Axford also provided financial support.

I would also like to thank Doctor Ron Kirkpatrick for his help and insight into the role of induced scattering in physica? situations of interest. Many useful discussions were had with Jonn

Pedicini and Harold Trease involving the techniques employed in this thesis. 


\section{REFERENCES}

1. S. Chandrasekhar, Radiative Transfer, ( Dover Publications, New York, 1960)

2. G. Magelssen, "Radiation Transport within Laser Fusion Targets", University of Wisconsin, UWFDM-215, (July 1977)

3. P. M. Campell, J. J. Kubis, and D. Mitrovich, "Kadiative Processes in a Laser Fusion Plasma", KMS Fusion, KMSF-U457, (Jan. 1976)

4. A. Sgro, "A Brief Description of the Ceres Photonics Package", Office Memorandum, LASL, TD-1, (Feb.26, 1974)

5. B. Freeman and C. G. Davis, "Numerical Solution of the Transport Equation", LASL, AFWL-TR-66-143, (1966)

6. R. P. Feynman, R. B. Leighton, and M. Sands, The R. P. Feynman Lectures on Physics, (Addison-Wesley Publishing Company, Reading Mass., 1966)

7. P. M. Campbell and J. J. Kubis, "A Variable Eddington Method for Radiation Transpori in Dense Fusion Plasmas", KMS Fusion, KMSF-U458, (1976)

8. G. N. Minerbo, "Maximura Entropy Eddington Factors", LASL, LA-6882-MS, (1977)

9. G. C. Pomraning, The Equations of Radiation Hydrodynamics, (Pergamon Press, New York, $\overline{1973 \text { ) }}$

10. A. S. Kompaneets, "The Escablishment of Thermal Equilibrium Between Quanta and Electrons", Sov. Phys. JETP, 31, 876-885, (Nov. 1956)

11. Ya. B. Zel'dovich and E. V. Levich, "Bose Condensation and Shock Waves in Photon Spectra", Sov. Phys. JETP, 55, 2423-2429, (Dec. 1968)

12. J. Coste and J. Peyraud, "Kinetics of the Compton Scatcering and the Bose Condensation of a Photon Gas", Phys. Rev. A, 12, 5. (Nov. 1975)

13. Ya. B. Zel'dovich, E. V. Levich, and R. A. Syunyaev, "Stimulated Compton Interaction Between Maxwellian Electrons and Spectrally Narrow Radiation", Sov. Phys. JETP, 62, 1392-1408, (Apr. 1972) 
14. C. Montes, "Photon Soliton and Fine Structure due to Nonlinear Compton Scattering", Phys. Rev. A, 19 24-42, (1979)

15. A. A. Galeev and R. A. Syunyaev, "Plasma Effects in Stimulated Compton Interaction Between Matter and Radiation", Sov. Phys. JETP, 63, 1266-1281, (Uct. 1972)

16. E. C. Zachmanoglou and D. W. Thoe, Introduction to Partial Differential Equations with Applications, (Williams and Wilkens Co., Baltimore, 1976)

17. O. Klein and Y. Nishina, "Uber die Streuung von Strahlung durch freie Elektronen nach der neuen relativistischen Quantendynamik von Dirac", Zs. f. Phys., 52, 853-868, (1929)

18. G. C. Pomraning, "Lorentz Transformation of the Equation of Radiative Transfer-The Scattering Kernel for Compton and Inverse Compton Processes", Gulf General Atomic, GA-9035, (Nov. 1968)

19. G. S. Fraley, "The Integrated Compton Cross Section and its Use in a Monte Carlo Scheme", LASL, LA-4592, (Apr. 1971)

20. S. Stone and R. G. Nelson, "Compton Scattering frum Relativistic Electrons", UCRL-14918-T, (May 1966)

21. L. Matteson, G. C. Pomraning, and H. L. Wilson, "Tabulation of the Compton and Inverse Compton Scattering Cross Section", Gulf General Atomic, GA-9694, (Aug. 1969)

22. J. P. Apruzese and J. Davis, "Evaluation of the Radiation Field in a Spherically Symmetric Plasma", NRL Memorandum report 3277, (May 1976)

23. W. H. Tucker, Radiation Processes in Astrophysics, (MIT Press, Cambridge, Massachusetts, 1975)

24. F. F. Chen, Introduction to Plasma Physics, (Plenum Press, New York, 1974)

25. Ya. B. Zel'dovich and R. A. Syunyaev, "Shock Wave Structure in the Radiation Spectrum During Bose Condensation of Photons", Zh. Eksp. Teor. Fiz., 62, 153-160, (Jan. 1972)

26. W. Heitler, The Quantum Theory of Radiation, (Clarendon Press, Oxford, 1954)

27. M. Abramowitz and I. A. Stegun, Handbook of Mathematical Functions, (Dover Press, New York, 1965)

28. I. S. Gradshteyn and I. M. Ryzhik, Table of Integrals Series and Products, (Academic Press, New York, 1965) 
29. J. Peyraud, "Theorie Cenetique Des Plasmas, Interaction Matiere-Rayonnement-I. Action D'un Rayonnement Donne Sur la Fonction de Distribution Electronique par L'Intermediaire des Chocs Compton", Le Journ. de Phys., 29, 88-96, (Jan. 1968)

30. J. Peyraud, "II. Action D'une Distribution Electronique Donnee sur le Rayonnement par L'intermediaire des Chocs Compton", Le Journ. de Phys., 29, 306-310, (Apr. 1968)

31. J. Peyraud, "III. Interaction Entre un Rayonnement Incense et un Plasma par Effet Compton Induit", Le Journ. de Phys., 29, 872-876, (Oct. 1968)

32. A. R. Fraser, "The Fundamental Equations of Kadiation Hydrodynamics", AWRE report, 0-82/65, (1965)

33. A. R. Fraser, "Radiative Transfer in Moving Media", AWRE report, $0-77 / 58,(1977)$

34. J. G. Eo11, "Note Concerning the Equations of Radiation Hydrodynamics", LASL, LA-UR-77-1408, (1977)

35. L. H. Thomas, "The Radiation Field of a Fluid in Motion", Quart. J. Math., 1, 239, (1930)

36. S. H. Hsieh and E. A. Spiegel, "The Equations of Photohydrodynamics", Astrophys. J., 207, 244-252, (July 1976)

37. V. M. Charugin and Yu. P. Ochelkov, "Omnidirectional Induced Compton Scattering by Relativistic Electrons", Astrophys. Space. Sci., 26, 337-344, (1974)

38. C. Montes, "Variability of Intensity of Interstellar Maser Lines due to Induced Compton Scattering", Astrophys. J., 216, 329-345, (Aug. 1977)

39. G. H. B. Thompson, Physics of Semiconductor Laser Devices, (John Wiley and Sons, New York, 1980)

40. L. L. Carter and F. D. Cashwell, Particle-Transport Simulation with the Monte Carlo Method, U. S. Critical Review Series, (1975)

41. G. C. Pomraning, "Maximum Entropy Eddington Factors and Flux Limited Diffusion Theory", J. Quan. Spec. Rad. Trans., 9, 407, (1969)

42. G. I. Bell and S. Glasstone, Nuclear Reactor Theory, (Van Nostrand Reinold, New York, 1970). 
43. R. D. Richtmyer and K. Morton, Difference Method ior Initial-Value Problems, (Interscience Publishers Inc., Neb Yurs, 1957).

44. G. N. Minerbo, Private communication (1980)

45. P. A. Stark, Introduction to Numerical Methods, (The Midimillitis Company, New York, 1970). 


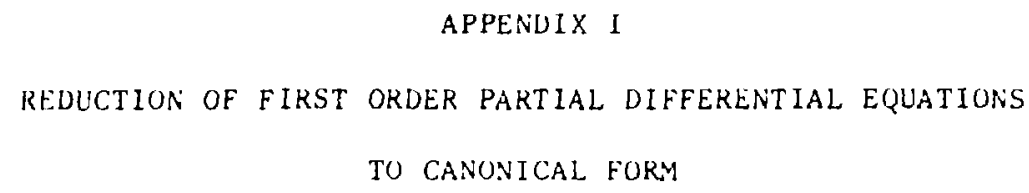

TO CANONICAL FORM

An outline of the techniques used to reduce sels of first order partial differential equations to canonical form is given in tais appendix.(16) First consider the set of $m$ first order partial ditferential equations of the form,

$$
\frac{\partial u_{i}}{\partial t}+\sum_{j=1}^{m} a_{i j}(x, t) \frac{\partial u_{j}}{\partial x}+b_{i}\left(x, t, u_{1}, u_{2}, \ldots, u_{m}\right)=0 \quad(I-1)
$$

for $i=1,2, \ldots, m$. This set of equations can be rewritten in vector from as

$$
\frac{\partial u}{\partial t}+A(x, t) \frac{\partial u}{\partial x}+\underline{b}(x, t, \underline{u})=\underline{0}
$$

where

$$
\underline{u}=\left(\begin{array}{c}
u_{1} \\
u_{2} \\
- \\
\cdot \\
u_{m}
\end{array}\right) \text {, }
$$




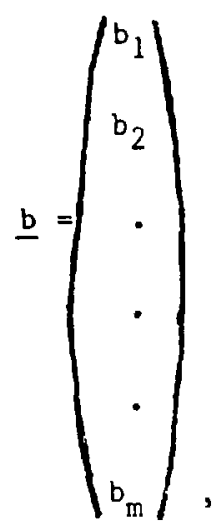

and

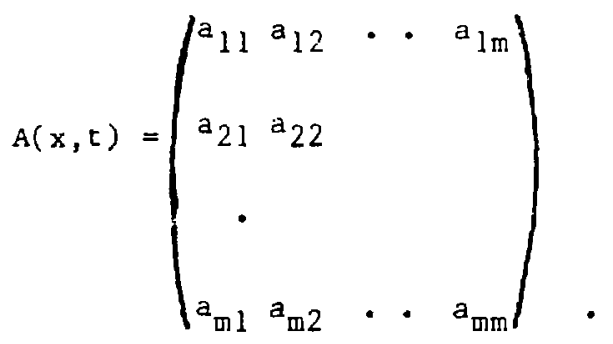

$(I-5)$

Now, let $A(x, \tau)$ have the eigenvalues $\lambda_{i}$ determined by the roots of the equation

$$
\operatorname{det}|A-\lambda \hat{I}|=0 \text {, }
$$

where $\hat{I}$ is the $m x$ m identity matrix. Also, let A have the eigenvectors $P_{i}$ determined by the aquations

$A P_{1}=\lambda_{1} P_{1}$ 
If there exist $m d i s t i n c t$ and real nonzero eigenvalues, $\lambda_{i}$, for $i=1,2, \ldots, m$ determined by Eq. $(1-6)$, then the set of Eqs. (I-l) are said to be hyperbolic.(16) For a hyperbolic set tinere are $m$ real and distinct eigenvectors $\underline{P}_{i}$.

We now define an $m \times m$ matrix $P$ such that the columas of the matrix are the eigenvectors $\underline{p}_{i}$ where

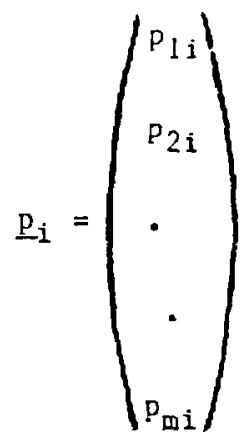

and

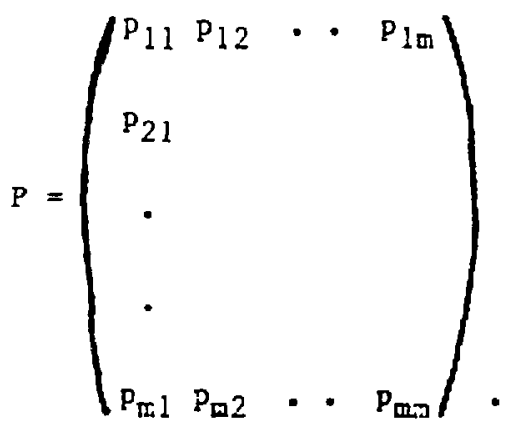


We also define a matrix $\Lambda$ whose diagonal is made up of the eigenvalues $\lambda_{i}$.

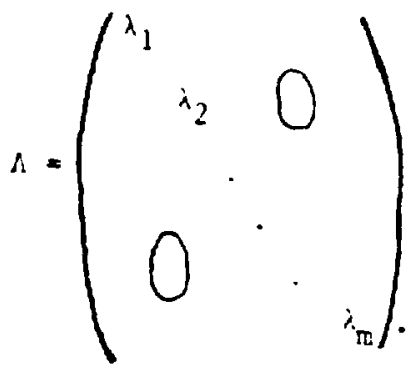

$1:-!$

New dependent variables are formed irow ile relais.

$\underline{v}=P^{-1} \underline{\underline{L}}$

$(:-: a)$

In this case we have

$$
\underline{u}=F \underline{v} \text {, }
$$

where

$$
\frac{\partial u}{\partial t}=P \frac{\partial v}{\partial t}+\frac{\partial P}{\partial t} \underline{v}
$$

and

$$
\frac{\partial u}{\partial x}=P \frac{\partial v}{\partial x}+\frac{\partial P}{\partial x} \underline{v} .
$$


Also note that

$\mathrm{P}^{-1} \dot{A} \mathrm{P}=\Lambda$.

$(1-15)$

Substituting these expressions into Eq. (1-2) yields

$$
\begin{aligned}
P \frac{\partial \underline{v}}{\partial t} & +A P \frac{\partial \underline{v}}{\partial x}+\frac{\partial P}{\partial t} \underline{v}+A \frac{\partial P}{\partial x} \underline{v} \\
& +\underline{b}(x, t, P \underline{v})=\underline{U} .
\end{aligned}
$$

If we ruitiply Eq. $(I-16)$ by $p^{-1}$ we obtain

$$
\frac{\partial \underline{y}}{\partial t}+\Lambda(x, t) \frac{\partial \underline{y}}{\partial x}+\underline{c}(x, t, \underline{v})=\underline{0} .
$$

In Eq. $(1-17)$ we have defined

$$
\begin{aligned}
\underline{c}(x, t, \underline{v}) & =P^{-1}\left(\frac{\partial P}{\partial t}+A \frac{\partial \underline{p}}{\partial x}\right) \underline{v} \\
& +P^{-1} \underline{D}(x, \tau, P \underline{v}) .
\end{aligned}
$$

Equations (I-17) and (I-18) give the canonical form of Eq. (1-2) in terms of the new canonical variables $\underline{v}$. These equations 
can now be solved via the method of characteristics. Solutions can be found at intersection points of the characteristic curves defined by

$$
\frac{d x_{i}(t)}{d t}=\lambda_{i}\left(x_{i}(t), t\right)
$$


APPENDIX II

EVALUATION OF FREQUENCY INTEGRALS

The frequency integrals encountered in this thesis were integrated numerically using the trapezoid rule for numerical integration. (45) This is accomplished in the following manner.

The integral we wish to evaluate can be written as

$$
F=\underbrace{G}_{v_{1}} d v f(v)
$$

In this case, the frequency range, $v_{1}<v<v_{G}$, has been divided into $G$ frequency points $v_{i}$ for $i=1,2, \ldots G$. It is assumed that $f\left(v_{i}\right)$ is known for $i=1,2, \ldots G$. The integral in Eq. (II-1) is now evaluated using the formula

$$
F=\sum_{i=2}^{G} \frac{1}{2}\left[f\left(v_{i}\right)+f\left(v_{i-1}\right)\right]\left(v_{i}-v_{i-1}\right) .
$$

The frequency point spacing used in this thesis was geometric. If an arithmetic spacing is used, numerical problems arise. If the frequency step makes a large jump, e.g., order of magnitude, a numerlcal Instability occurs at the points where the jump occurs. A geometric spacing does not have this problem. An example of geometric spacing was discussed in Chapter Four where each frequency step was incremented by a factor of 1.087. An example of an arithematic spacing with a large frequency step 
jump is the following. If the frequency range from looev to $1000 \mathrm{eV}$ is incremented by 10 steps each being $100 \mathrm{eV}$ is size, and the range from $1000 \mathrm{eV}$ to $10000 \mathrm{eV}$ is incremented by 10 steps each being $1000 \mathrm{eV}$ in size, a large jump occurs at $1000 \mathrm{eV}$. It is at these points that instabilities occured.

Several remarks need to be made concerning the upper and lower limits of integration shown in Eq. (II-l). Although the lower limit should actually be zero, scattering below the lowest frequency group is negligible. Hence, the lower limit used is that of the lowest frequency group. This value is usually je!. Again, scatering above the highes frequency group may be neglected, and, consequently, this value is used as the uppe: limit of integration.

The error introduced by use of the trapezoid rule may be calculated from $(45)$

$$
E=\sum_{i=2}^{G} \frac{\left(v_{i}-v_{i-1}\right)^{3}}{12} \frac{d^{2} f}{d v^{2}}\left(v_{i}\right)
$$

This error is not significant since $f^{\prime \prime}(v)$ is extremely small in all cases. 


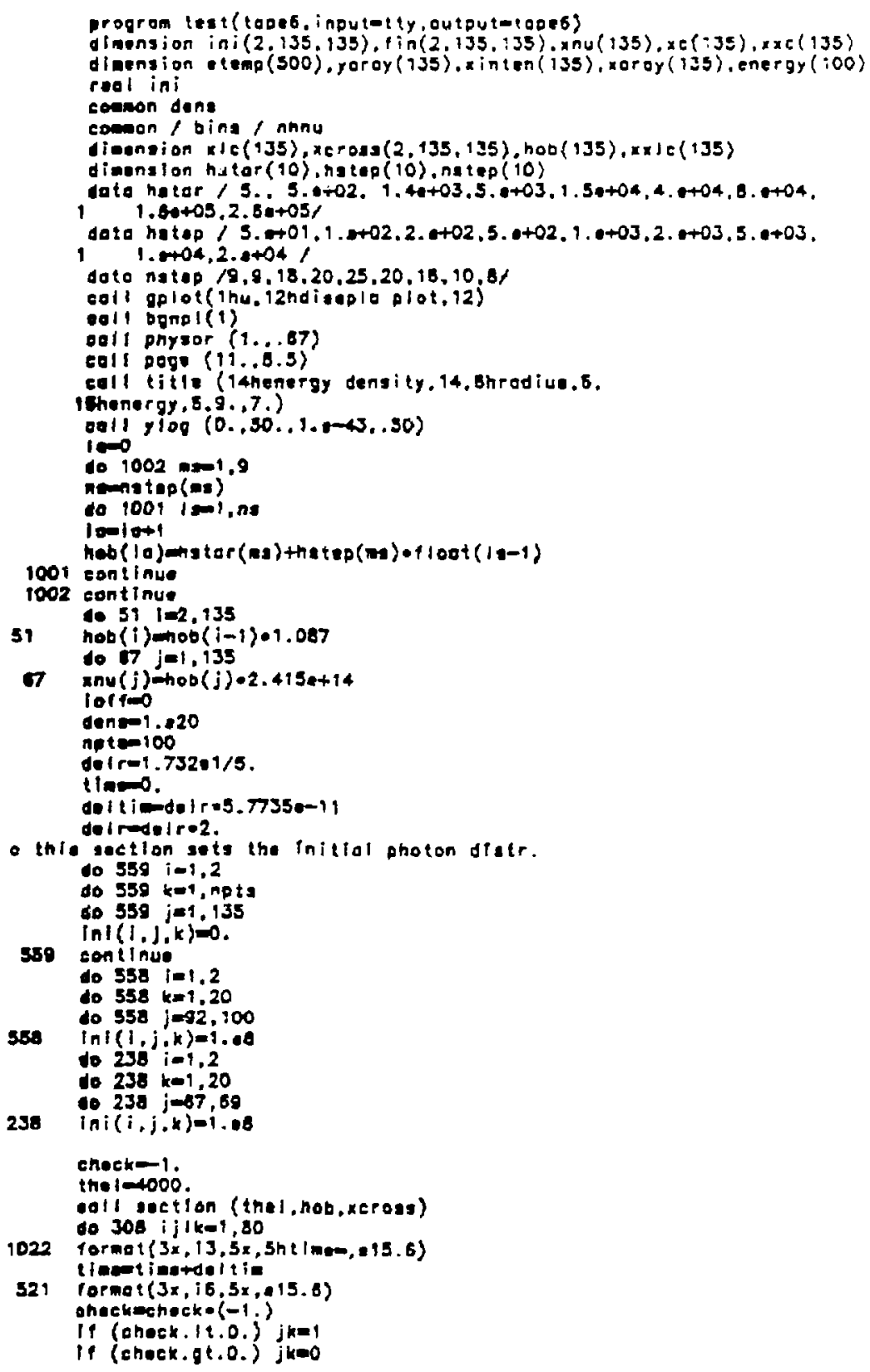




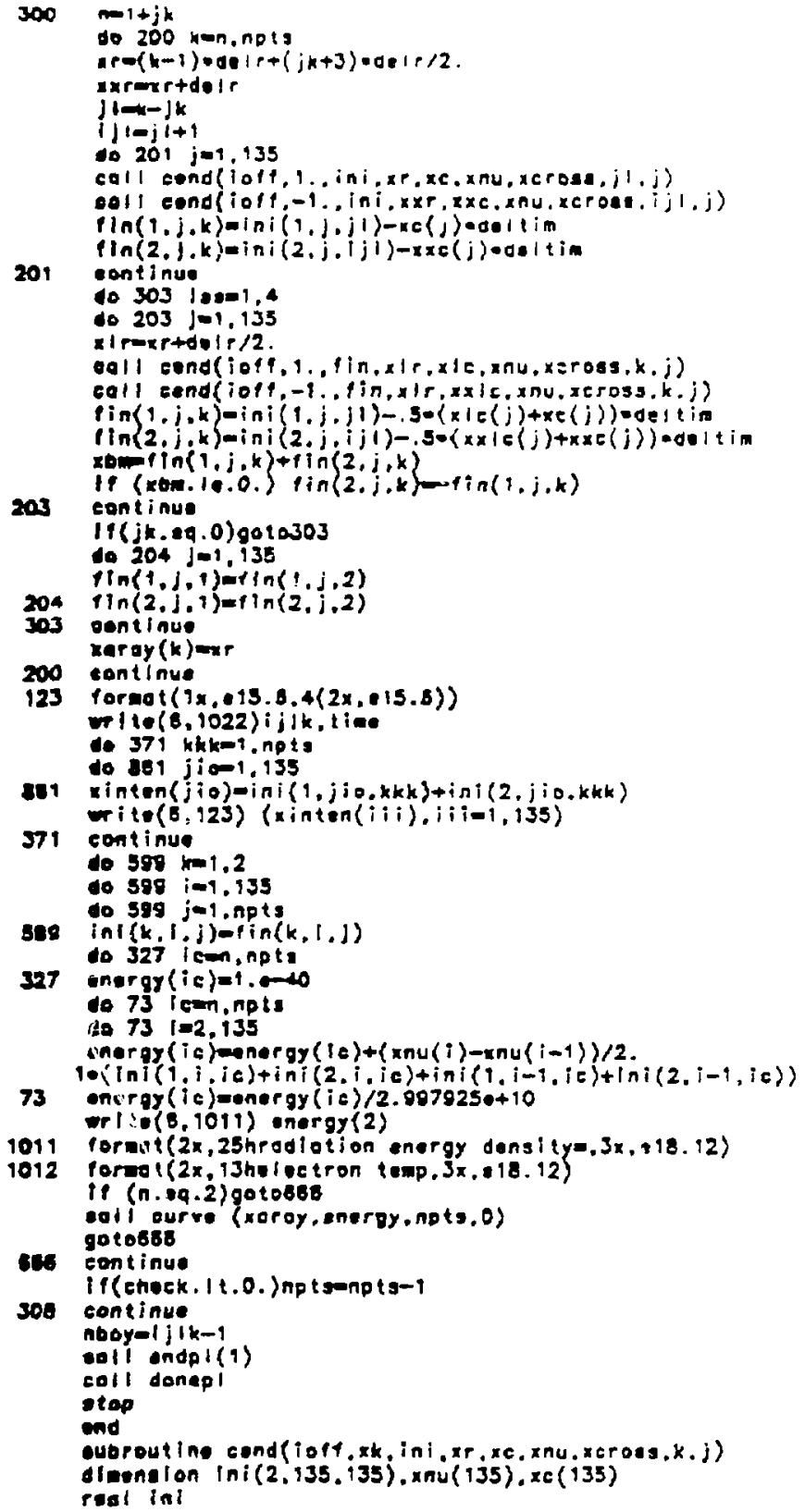




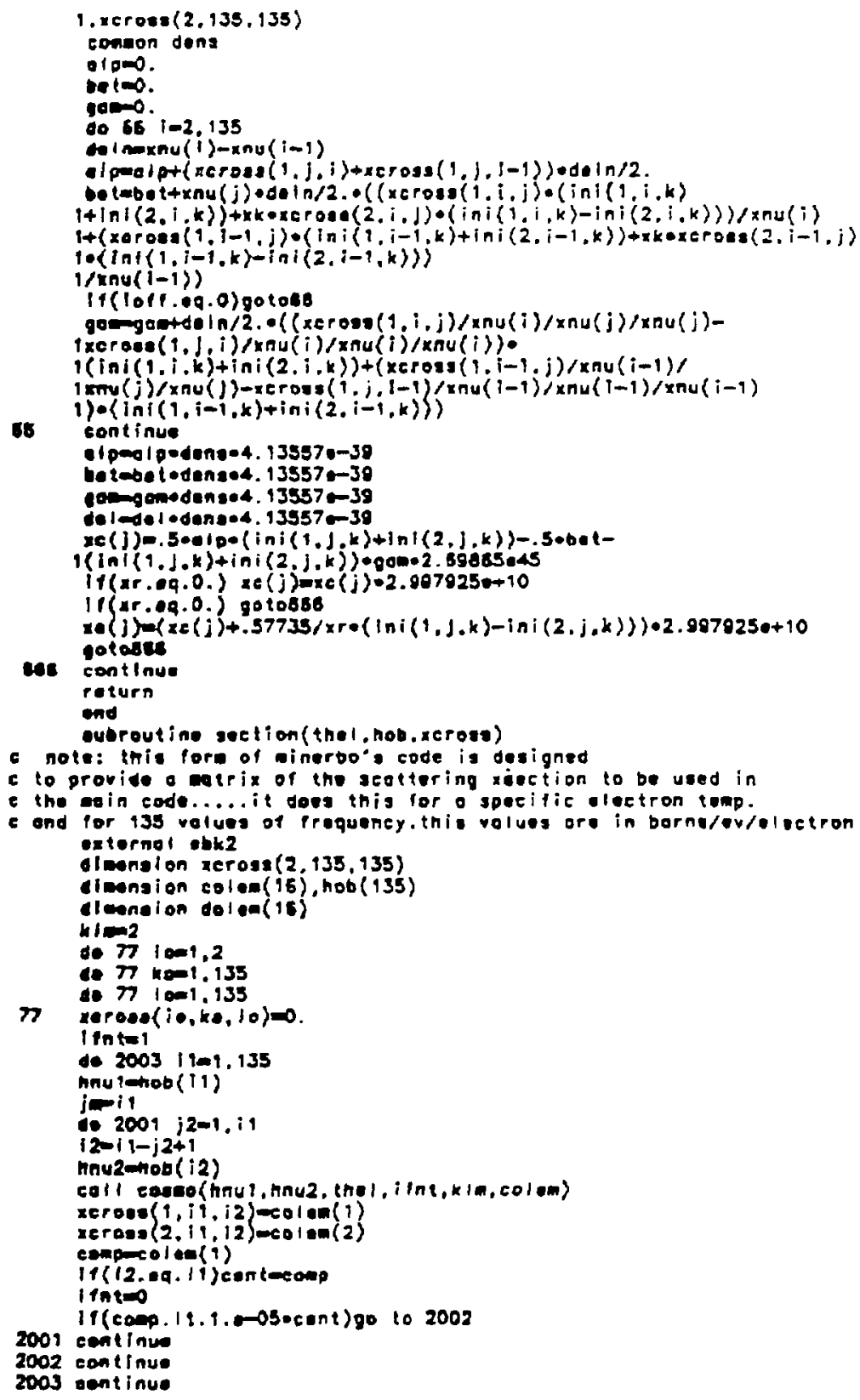




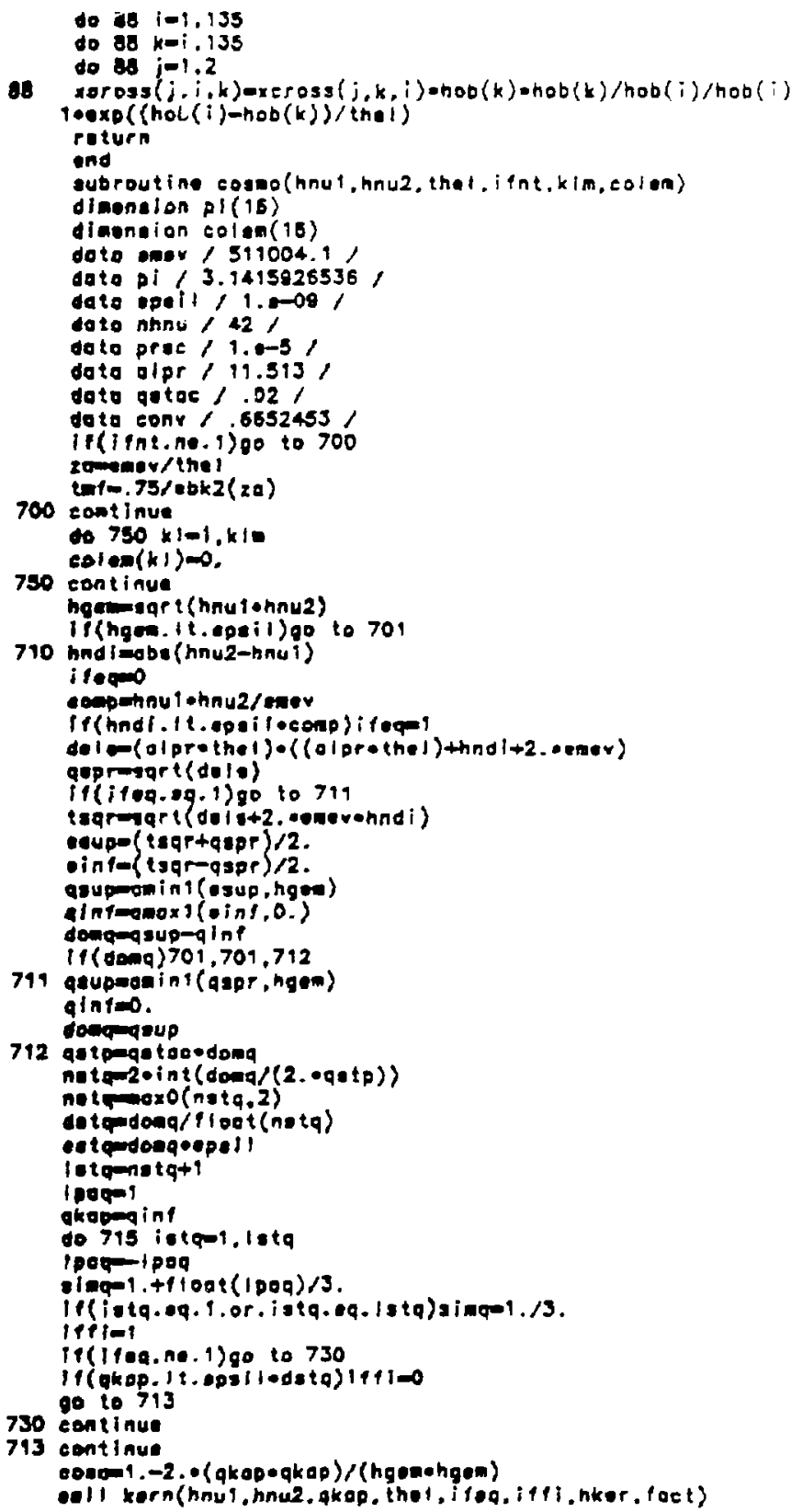




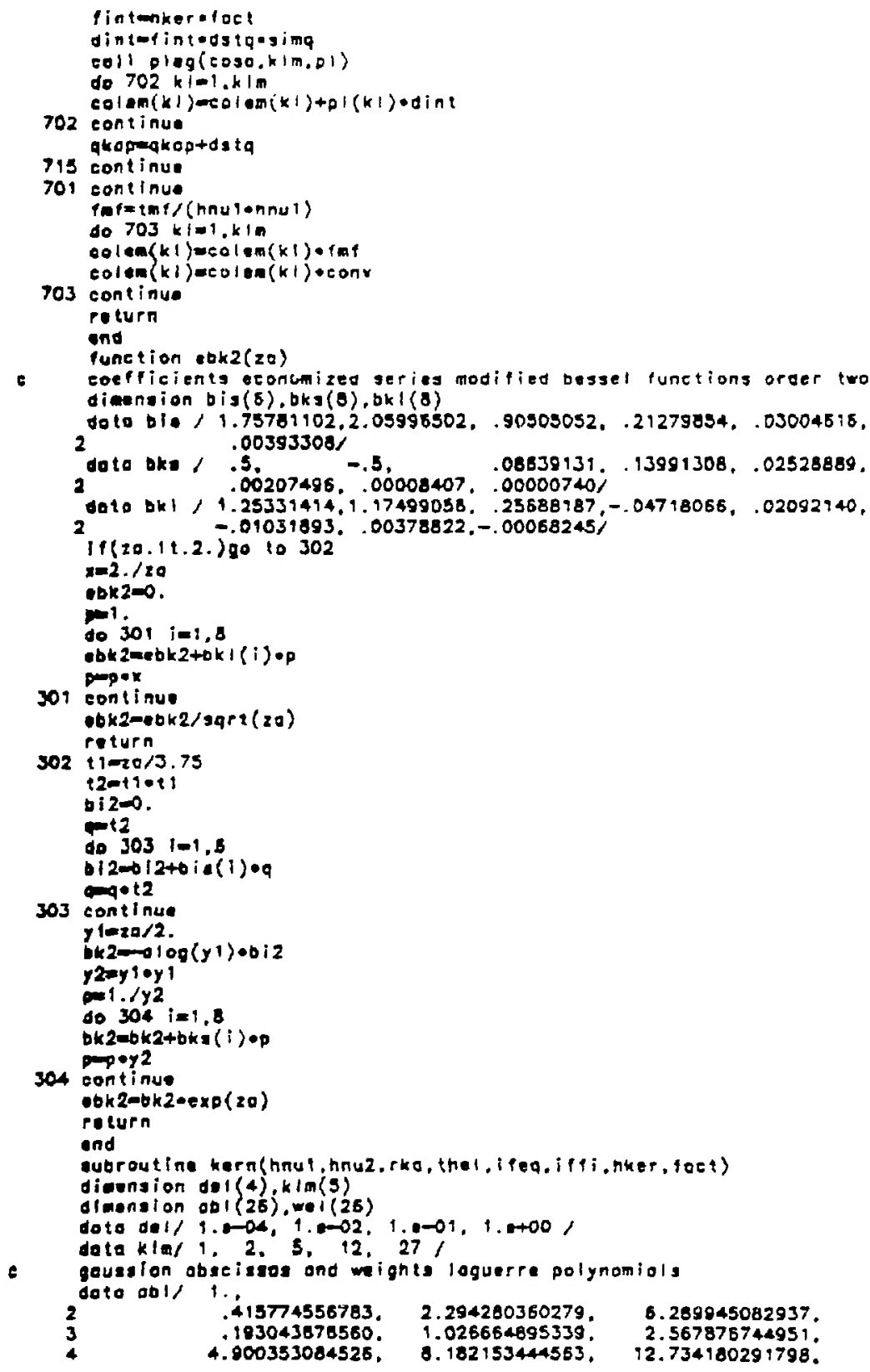




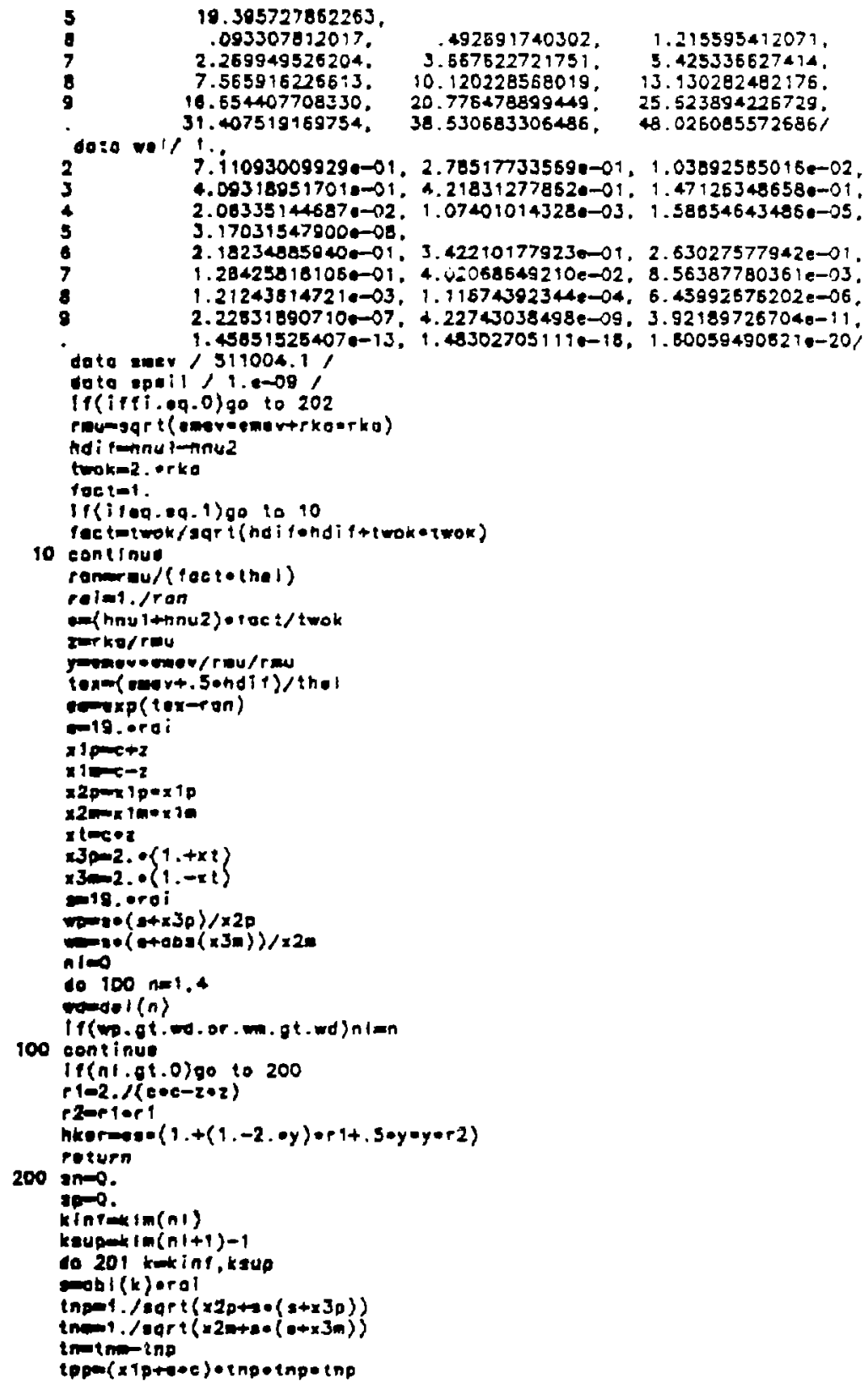




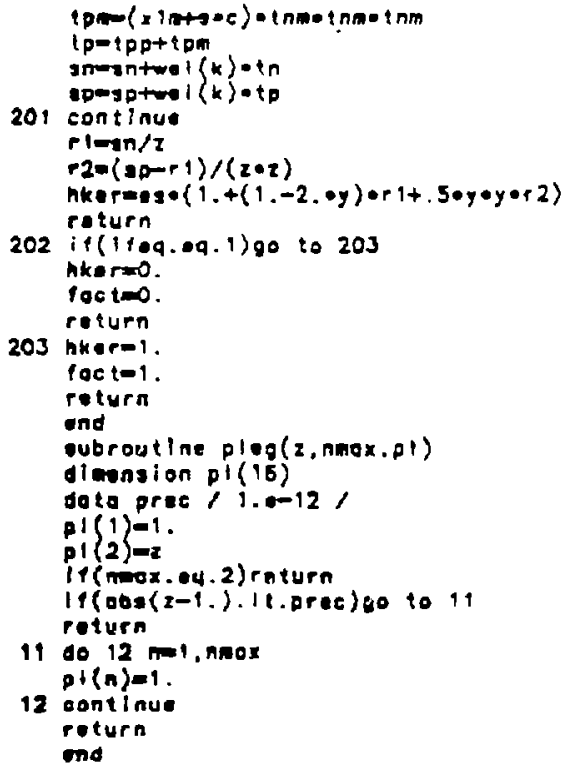

